\title{
Labour market
}

\section{Labour Force Survey}

(Tables 7.1-7.3, 7.6, 7.9, 7.10-7.11, 7.13 and 7.16-7.18)

\section{Labour Force Survey}

\section{Background}

The Labour Force Survey (LFS) is the largest regular household survey in the UK, with interviews being conducted continuously throughout the year. In any three-month period, a nationally representative sample of approximately 110,000 people, aged 16 and over, in around 50,000 households are interviewed. Each household is interviewed five times, at three-monthly intervals. The initial interview is done faceto-face by an interviewer visiting the address, except for residents north of the Caledonian Canal in Scotland. The other interviews are done by telephone wherever possible. The survey asks a series of questions about respondents' personal circumstances and their labour market activity. Most questions refer to activity in the week before the interview.

The LFS collects information on a sample of the population. To convert this information to give estimates for the population the data must be grossed. This is achieved by calculating weighting factors (often referred to simply as weights) which can be applied to each sampled individual in such a way that the weighted-up results match estimates or projections of the total population in terms of age distribution, sex and region of residence. There is a considerable amount of ongoing research to improve methodologies. Whenever methodologies are implemented the estimates may be revised.

The concepts and definitions used in the LFS are agreed by the International Labour Organisation (ILO) - an agency of the United Nations. The definitions are used by European Union member countries and members of the Organisation for Economic Co-operation and Development (OECD).

The LFS was carried out every two years from 1973 to 1983. The ILO definition was first used in 1984. This was also the first year in which the survey was conducted on an annual basis with results available for every spring quarter (representing an average of the period from March to May). The survey moved to a continuous basis in spring 1992 in Great Britain and in winter 1994/95 in Northern Ireland, with average quarterly results published four times a year for seasonal quarters: spring (March to May), summer (June to August), autumn (September to November) and winter (December to February). From April 1998, results are published 12 times a year for the average of three consecutive months.

\section{Strengths and limitations of the LFS}

The LFS produces coherent labour market information on the basis of internationally standard concepts and definitions. It is a rich source of data on a wide variety of labour market and personal characteristics. It is the most suitable source for making comparisons between countries. The LFS is designed so that households interviewed in each three-month period constitute a representative sample of UK households. The survey covers those living in private households and nurses in National Health Service accommodation. Students living in halls of residence have been included since 1992 as information about them is collected at their parents' address.

However the LFS has its limitations. It is a sample survey and is therefore subject to sampling variability. The survey does not include people living in institutions such as hostels, hotels, boarding houses, mobile home sites or residential homes. 'Proxy' reporting (when members of the household are not present at the interview and another member of the household answers the questions on their behalf) can affect the quality of information on topics such as earnings, hours worked, benefit receipt and qualifications. Around a third of interviews are conducted by proxy, usually by a spouse or partner but sometimes by a parent or other near relation. LFS estimates are also potentially affected by non-response.

\section{Sampling Variability}

Survey estimates are prone to sampling variability. The easiest way to explain this concept is by example. In the September to November 1997 period, ILO unemployment in Great Britain (seasonally adjusted) stood at $1,847,000$. If we drew another sample for the same period we could get a different result, perhaps $1,900,000$ or $1,820,000$.

In theory, we could draw many samples, and each would give a different result. This is because each sample would be made up of different people who would give different answers to the questions. The spread of these results is the sampling variability. Sampling variability is determined by a number of factors including the sample size, the variability of the population from which the sample is drawn and the sample design. Once we know the sampling variability we can calculate a range of values about the sample estimate that represents the expected variation with a given level of assurance. This is called a confidence interval. For a 95 per cent confidence interval we expect that in 95 per cent of the samples (19 times out of 20) the confidence interval will contain the true value that would be obtained by surveying the entire population. For the example given above, we can be 95 per cent confident that the true value was in the range $1,791,000$ to $1,903,000$. 


\section{Unreliable estimates}

Estimates of small numbers have relatively wide confidence intervals making them unreliable. For this reason, ONS does not currently publish LFS estimates below 10,000.

\section{Non-response}

All surveys are subject to non-response - that is respondents in the sample who either refuse to take part in the survey or who cannot be contacted. Non-response can introduce bias to a survey, particularly if the people not responding have characteristics that are different from those who do respond.

The LFS has a response rate of around 65 per cent to the first interview, and over 90 per cent of those who are interviewed once go on to complete all five interviews. These are relatively high levels for a household survey.

Any bias from non-response is minimised by weighting the results. Weighting (or grossing) converts sample data to represent the full population. In the LFS, the data are weighted separately by age, sex and area of residence to population estimates based on the census. Weighting also adjusts for people not in the survey and thus minimises non-response bias.

\section{LFS concepts and definitions}

Discouraged worker - A sub-group of the economically inactive population who said although they would like a job their main reason for not seeking work was because they believed there were no jobs available.

Economically active - People aged 16 and over who are either in employment or unemployed.

Economic activity rate - The number of people who are in employment or unemployed expressed as a percentage of the relevant population.

Economically inactive - People who are neither in employment nor unemployed. These include those who want a job but have not been seeking work in the last four weeks; those who want a job and are seeking work but are not available to start; and those who do not want a job.

Employment - People aged 16 and over who did at least one hour of paid work in the reference week (as an employee or self-employed); those who had a job that they were temporarily away from; those on government-supported training and employment programmes; and those doing unpaid family work.
Employees - The division between employees and self employed is based on survey respondents' own assessment of their employment status.

Full-time - The classification of employees, self-employed and unpaid family workers in their main job as full-time or part-time is on the basis of self-assessment. However, people on government supported employment and training programmes that are at college in the reference week are classified, by convention, as part-time.

Government-supported training and employment programmes - Comprise all people aged 16 and over participating in one of the government's employment and training programmes (Youth Training, Training for Work and Community Action), together with those on similar programmes administered by Training and Enterprise Councils in England and Wales, or Local Enterprise Companies in Scotland.

Hours worked - Respondents to the LFS are asked a series of questions enabling the identification of both their usual hours and their actual hours. Total hours include overtime (paid and unpaid) and exclude lunch breaks.

- Actual hours worked - Actual hours worked statistics measure how many hours were actually worked. These statistics are directly affected by changes in the number of people in employment and in the number of hours that individual works

- Usual hours worked - Usual hours worked statistics measure how many hours people usually work per week. Compared with actual hours worked, they are not affected by absences and so can provide a better measure of normal working patterns

Unemployment - The number of unemployed people in the UK is measured through the LFS following the internationally agreed definition recommended by the International Labour Organisation (ILO), an agency of the United Nations.

Unemployed people who are:

1. without a job, have actively sought work in the last four weeks and are available to start work in the next two weeks or

2. out of work, have found a job and are waiting to start in the next two weeks

Unemployment (rate) - The number of unemployed people expressed as a percentage of the relevant economically active population. 
Unemployment (duration) - The duration of a respondents' unemployment is defined as the shorter of the following two periods:

- duration of active search for work

- length of time since employment

Part-time - see full-time.

Second jobs - Jobs which LFS respondents hold in addition to a main full-time or part-time job.

Self-employment - See Employees.

Temporary employees - In the LFS these are defined as those employees who say that their main job is nonpermanent in one of the following ways: fixed period contract, agency temping, casual work, seasonal work or other temporary work.

Unpaid family workers - Persons doing unpaid work for a business they own or for a business that a relative owns.

\section{International Employment Comparisons}

\section{(Table 7.7)}

All employment rates for European Union (EU) countries published by Eurostat (including the rate for the UK) are based on the population aged 15-64. The rates for Canada and Japan are also based on the population aged 15-64, but the rate for the US is for those aged 16-64. The employment rate for the UK published by ONS is based on the working age population aged 16-64 (men) and 16-59 (women) and

therefore takes into account both the current school leaving age and state pension ages.

The unemployment rate published by Eurostat for most EU countries (but not for the UK), are calculated by extrapolating from the most recent LFS data using monthly registered unemployment data. A standard population basis (15-74) is used by Eurostat except for Spain and the UK (16-74). The unemployment rate for the US is based on those aged 16 and over, but the rates for Canada and Japan are for those aged 15 and over. All unemployment rates are seasonally adjusted.

The unemployment rate for the UK published by Eurostat is based on the population aged 16-74 while the unemployment rate for the UK published by ONS is based on those aged 16 and over. There are other minor definitional differences.

\section{Jobseekers allowance claimant count}

\section{(Tables 7.14 and 7.15)}

This is a count of all people claiming Jobseeker's Allowance (JSA) at Jobcentre Plus local offices. People claiming JSA must declare that they are:

- out of work

- capable of work

- $\quad$ available for work

- $\quad$ actively seeking work

during the week in which the claim is made.

All people claiming JSA on the day of the monthly count are included in the claimant count, irrespective of whether they are actually receiving benefits. Also see table 10.6 in Social protection chapter.

\section{Labour disputes}

(Table 7.19)

These figures exclude details of stoppages involving fewer than ten workers or lasting less than one day, except any in which the aggregate number of working days lost is 100 or more. There may be some under-recording of small or short stoppages; this would have much more effect on the total of stoppages than of working days lost. Some stoppages which affected more than one industry group have been counted under each of the industries but only once in the totals. Stoppages have been classified using the Standard Industrial Classification (SIC) 2003.

The figures for working days lost and workers involved have been rounded and consequently the sum of the constituent items may not agree with the totals. Classifications by size are based on the full duration of stoppages where these continue into the following year. Working days lost per thousand employees are based on the latest available mid-year (June) estimates of employee jobs.

\section{Annual Survey of Hours and Earnings}

(Tables 7.20, 7.21, 7.24 and 7.25)

The Annual Survey of Hours and Earnings (ASHE) is based on a one per cent sample of employee jobs taken from HM Revenue \& Customs (HMRC) PAYE records. Information on earnings and paid hours worked is obtained from employers and treated confidentially. ASHE does not cover the 
self-employed nor does it cover employees not paid during the reference period.

The headline statistics for ASHE are based on the median rather than the mean. The median is the value below which 50 per cent of employees fall. It is ONS's preferred measure of average earnings as it is less affected by a relatively small number of very high earners and the skewed distribution of earnings. It therefore gives a better indication of typical pay than the mean.

The earnings information presented relates to gross pay before tax, National Insurance or other deductions, and excludes payments in kind. With the exception of annual earnings, the results are restricted to earnings relating to the survey pay period and so exclude payments of arrears from another period made during the survey period; any payments due as a result of a pay settlement, but not yet paid at the time of the survey, will also be excluded.

More detailed information is available on the ONS website at: www.statistics.gov.uk/StatBase/Product.asp?vlnk=13101

- to increase the estimates of the level of average weekly pay over estimates published from the NES

- for males the increase in estimates of earnings is more than the increase for females. In particular this affects hourly pay excluding overtime, which is used in the calculation of ONS's preferred measure of the gender pay gap. The estimate of hourly pay for males is increased more then the estimate for females, which widens the estimate of the gap between male and female hourly pay

- $\quad$ estimates of the level of earnings for people working in London are increased more than estimates for other regions. This widens the estimate of the difference in pay between London and other regions of the UK

For information about methodological changes to the 2007 ASHE survey see: www.statistics.gov.uk/downloads/theme_ labour/ASHE/ChangelnASHE07.pdf

\section{Trade unions}

\section{(Table 7.26)}

The statistics relate to all organisations of employees known to the Certification Officer with head offices in the UK that fall within the appropriate definition of a trade union in the Trade Union and Labour Relations (Consolidation) Act 1992. Included in the data are home and overseas membership figures of contributory and non-contributory members. Employment status of members is not provided and the figures may therefore include some people who are selfemployed, unemployed or retired. 
Labour Market

At Quarter 2 each year². Seasonally adjusted

Thousands and percentages

\begin{tabular}{|c|c|c|c|c|c|c|c|c|c|}
\hline & $\begin{array}{r}\text { All aged } 16 \& \\
\text { over }\end{array}$ & $\begin{array}{r}\text { Total } \\
\text { economically } \\
\text { active }\end{array}$ & $\begin{array}{r}\text { Total in } \\
\text { employment }\end{array}$ & $\begin{array}{r}\text { Total } \\
\text { unemployed }\end{array}$ & $\begin{array}{r}\text { Economically } \\
\text { inactive }\end{array}$ & $\begin{array}{r}\text { Economic } \\
\text { activity } \\
\text { rate(\%) }\end{array}$ & $\begin{array}{r}\text { Employment } \\
\operatorname{rate}^{2}(\%)\end{array}$ & $\begin{array}{r}\text { Unemployment } \\
\text { rate }^{3}(\%)\end{array}$ & $\begin{array}{r}\text { Economic } \\
\text { inactivity } \\
\text { rate }^{4}(\%)\end{array}$ \\
\hline & MGSL & MGSF & MGRZ & MGSC & MGSI & MGWG & MGSR & MGSX & YBTC \\
\hline 2002 & 46787 & 29450 & 27921 & 1529 & 17337 & 63.0 & 59.7 & 5.2 & 37.0 \\
\hline 2003 & 47087 & 29675 & 28186 & 1489 & 17411 & 63.0 & 59.9 & 5.1 & 37.0 \\
\hline 2004 & 47448 & 29909 & 28485 & 1424 & 17538 & 63.0 & 60.0 & 4.8 & 37.0 \\
\hline 2005 & 47871 & 30239 & 28774 & 1465 & 17632 & 63.2 & 60.1 & 4.9 & 36.8 \\
\hline 2006 & 48268 & 30698 & 29030 & 1669 & 17570 & 63.6 & 60.2 & 5.4 & 36.4 \\
\hline 2007 & 48668 & 30875 & 29222 & 1653 & 17793 & 63.5 & 60.0 & 5.3 & 36.5 \\
\hline 2008 & 49059 & 31220 & 29443 & 1776 & 17839 & 63.6 & 60.0 & 5.7 & 36.4 \\
\hline 2009 & 49468 & 31374 & 28979 & 2395 & 18093 & 63.5 & 58.6 & 7.6 & 36.5 \\
\hline \multicolumn{10}{|l|}{ Men } \\
\hline & MGSM & MGSG & MGSA & MGSD & MGSJ & MGWH & MGSS & MGSY & YBTD \\
\hline 2002 & 22600 & 16018 & 15099 & 920 & 6582 & 70.8 & 66.8 & 5.7 & 29.1 \\
\hline 2003 & 22775 & 16161 & 15262 & 900 & 6614 & 71.0 & 67.0 & 5.6 & 29.0 \\
\hline 2005 & 23214 & 16397 & 15535 & 862 & 6817 & 70.6 & 66.9 & 5.3 & 29.4 \\
\hline 2006 & 23438 & 16628 & 15662 & 966 & 6811 & 70.9 & 66.9 & 5.8 & 29.1 \\
\hline 2007 & 23668 & 16757 & 15813 & 944 & 6911 & 70.8 & 66.8 & 5.6 & 29.2 \\
\hline 2008 & 23891 & 16941 & 15894 & 1047 & 6950 & 70.9 & 66.5 & 6.2 & 29.1 \\
\hline 2009 & 24104 & 16962 & 15497 & 1465 & 7142 & 70.3 & 64.3 & 8.6 & 29.6 \\
\hline \multicolumn{10}{|c|}{ Women } \\
\hline & MGSN & MGSH & MGSB & MGSE & MGSK & MGWI & MGST & MGSZ & YBTE \\
\hline 2002 & 24186 & 13432 & 12823 & 609 & 10755 & 55.5 & 53.0 & 4.5 & 44.5 \\
\hline 2003 & 24311 & 13515 & 12925 & 590 & 10797 & 55.6 & 53.1 & 4.4 & 44.4 \\
\hline 2004 & 24469 & 13669 & 13080 & 589 & 10800 & 55.8 & 53.5 & 4.3 & 44.2 \\
\hline 2005 & 24657 & 13842 & 13239 & 603 & 10815 & 56.1 & 53.7 & 4.4 & 43.9 \\
\hline 2006 & 24830 & 14070 & 13367 & 704 & 10760 & 56.7 & 53.8 & 5.0 & 43.3 \\
\hline 2007 & 25001 & 14118 & 13409 & 709 & 10883 & 56.5 & 53.7 & 5.1 & 43.5 \\
\hline 2008 & 25168 & 14278 & 13549 & 729 & 10889 & 56.8 & 53.8 & 5.1 & 43.3 \\
\hline \multirow[t]{2}{*}{2009} & 25364 & 14412 & 13482 & 930 & 10952 & 56.8 & 53.1 & 6.4 & 43.2 \\
\hline & $\begin{array}{r}\text { All aged } 16 \text { to } \\
59 / 64\end{array}$ & $\begin{array}{r}\text { Total } \\
\text { economically } \\
\text { active }\end{array}$ & $\begin{array}{r}\text { Total in } \\
\text { employment }\end{array}$ & $\begin{array}{r}\text { Total } \\
\text { unemployed }\end{array}$ & $\begin{array}{r}\text { Economically } \\
\text { inactive }\end{array}$ & $\begin{array}{r}\text { Economic } \\
\text { activity } \\
\text { rate(\%) }\end{array}$ & $\begin{array}{r}\text { Employment } \\
\text { rate(\%) }\end{array}$ & $\begin{array}{r}\text { Unemployment } \\
\text { rate(\%) }\end{array}$ & $\begin{array}{r}\text { Economic } \\
\text { inactivity } \\
\text { rate }(\%)\end{array}$ \\
\hline \multicolumn{10}{|c|}{ People } \\
\hline & YBTF & YBSK & YBSE & YBSH & YBSN & MGSO & MGSU & YBTI & YBTL \\
\hline 2002 & 36304 & 28542 & 27034 & 1509 & 7762 & 78.6 & 74.5 & 5.3 & 21.4 \\
\hline 2003 & 36514 & 28713 & 27240 & 1472 & 7801 & 78.6 & 74.6 & 5.2 & 21.3 \\
\hline 2004 & 36773 & 28893 & 27486 & 1407 & 7880 & 78.6 & 74.7 & 4.9 & 21.4 \\
\hline 2005 & 37089 & 29149 & 27705 & 1445 & 7940 & 78.6 & 74.7 & 5.0 & 21.4 \\
\hline 2006 & 37365 & 29506 & 27863 & 1643 & 7859 & 78.9 & 74.6 & 5.6 & 21.0 \\
\hline 2007 & 37565 & 29625 & 27996 & 1629 & 7940 & 78.9 & 74.6 & 5.5 & 21.1 \\
\hline 2008 & 37743 & 29871 & 28120 & 1751 & 7872 & 79.1 & 74.5 & 5.9 & 20.9 \\
\hline 2009 & 37922 & 29956 & 27597 & 2359 & 7967 & 79.0 & 72.8 & 7.9 & 21.0 \\
\hline \multicolumn{10}{|l|}{ Men } \\
\hline & YBTG & YBSL & YBSF & YBSI & YBSO & MGSP & MGSV & YBTJ & YBTM \\
\hline 2002 & 18727 & 15712 & 14801 & 911 & 3015 & 83.9 & 79.0 & 5.8 & 16.1 \\
\hline 2003 & 18855 & 15823 & 14932 & 891 & 3032 & 83.9 & 79.2 & 5.6 & 16.1 \\
\hline 2004 & 19010 & 15894 & 15068 & 827 & 3116 & 83.6 & 79.3 & 5.2 & 16.4 \\
\hline 2005 & 19198 & 16027 & 15175 & 852 & 3171 & 83.5 & 79.0 & 5.3 & 16.5 \\
\hline 2006 & 19380 & 16228 & 15272 & 955 & 3152 & 83.7 & 78.8 & 5.9 & 16.3 \\
\hline 2007 & 19549 & 16341 & 15407 & 935 & 3208 & 83.6 & 78.8 & 5.7 & 16.4 \\
\hline 2008 & 19688 & 16487 & 15452 & 1035 & 3201 & 83.8 & 78.5 & 6.3 & 16.3 \\
\hline 2009 & 19807 & 16504 & 15055 & 1449 & 3304 & 83.3 & 76.0 & 8.8 & 16.7 \\
\hline \multicolumn{10}{|c|}{ Women } \\
\hline & YBTH & YBSM & YBSG & YBSJ & YBSP & MGSQ & MGSW & YBTK & YBTN \\
\hline 2002 & 17577 & 12830 & 12232 & 598 & 4747 & 73.0 & 69.6 & 4.6 & 27.0 \\
\hline 2003 & 17660 & 12890 & 12309 & 582 & 4769 & 73.0 & 69.7 & 4.5 & 27.0 \\
\hline 2004 & 17763 & 12999 & 12419 & 580 & 4765 & 73.2 & 69.9 & 4.4 & 26.8 \\
\hline 2005 & 17891 & 13123 & 12530 & 592 & 4769 & 73.3 & 70.0 & 4.5 & 26.7 \\
\hline 2006 & 17985 & 13278 & 12591 & 688 & 4707 & 73.8 & 70.0 & 5.2 & 26.1 \\
\hline 2007 & 18016 & 13284 & 12589 & 695 & 4732 & 73.8 & 69.9 & 5.3 & 26.3 \\
\hline 2008 & 18055 & 13384 & 12669 & 716 & 4671 & 74.1 & 70.2 & 5.3 & 25.8 \\
\hline 2009 & 18116 & 13452 & 12542 & 910 & 4664 & 74.3 & 69.2 & 6.8 & 25.7 \\
\hline
\end{tabular}

1 The Labour Force Survey (LFS) is a survey of the population of private 3 The headline unemployment rate is the number unemployed people (aged households, student halls of residence and NHS accommodation. $16+$ ) divided by the economically active population (aged 16+). The economi-

2 The headline employment rate is the number of working age people (aged cally active population is defined as those in employment plus those who are 16 to 59 for women and 16 to 64 for men) in employment divided by the unemployed.

working age population.

4 The headline inactivity rate is the number of working age inactive people (aged 16 to 59 for women and 16 to 64 for men) divided by the work population 


\begin{tabular}{|c|c|c|c|c|c|c|c|c|c|c|c|c|c|}
\hline & \multicolumn{6}{|c|}{ All in employment } & \multicolumn{2}{|c|}{ Total employment $^{1}$} & \multicolumn{2}{|c|}{ Employees $^{1}$} & \multicolumn{2}{|c|}{ Self-employed ${ }^{1}$} & \multirow[b]{2}{*}{$\begin{array}{r}\text { Workers } \\
\text { with second } \\
\text { jobs }\end{array}$} \\
\hline & Total & Employees & $\begin{array}{r}\text { Self } \\
\text { S employed }\end{array}$ & $\begin{array}{r}\text { Unpaid } \\
\text { family } \\
\text { workers }\end{array}$ & $\begin{array}{r}\text { Governm } \\
\text { suppor } \\
\text { train } \\
\text { employm } \\
\text { programn }\end{array}$ & $\begin{array}{l}\text { nent } \\
\text { rted } \\
\text { ning } \\
\text { and } \\
\text { nent } \\
\text { mes }\end{array}$ & Full-time & Part-time & Full-time & Part-time & Full-time & Part-time & \\
\hline \multicolumn{14}{|c|}{ People } \\
\hline & MGRZ & MGRN & MGRQ & MGRT & MG & RW & YCBE & YCBH & YCBK & YCBN & YCBQ & YCBT & YCBW \\
\hline 2002 & 27921 & 24386 & 3337 & 95 & & 103 & 20809 & 7112 & 18173 & 6213 & 2565 & 772 & 1150 \\
\hline 2003 & 28186 & 24427 & 3565 & 95 & & 100 & 20915 & 7271 & 18116 & 6311 & 2730 & 834 & 1116 \\
\hline 2004 & 28485 & 24645 & 3618 & 97 & & 124 & 21131 & 7354 & 18265 & 6381 & 2789 & 830 & 1073 \\
\hline 2005 & 28774 & 24929 & 3636 & 96 & & 115 & 21484 & 7290 & 18596 & 6333 & 2811 & 825 & 1058 \\
\hline 2006 & 29030 & 25098 & 3738 & 97 & & 97 & 21642 & 7388 & 18712 & 6386 & 2864 & 874 & 1053 \\
\hline 2007 & 29222 & 25204 & 3806 & 102 & & 110 & 21801 & 7422 & 18844 & 6360 & 2891 & 915 & 1097 \\
\hline 2008 & 29443 & 25407 & 3826 & 101 & & 110 & 21938 & 7505 & 18969 & 6438 & 2911 & 915 & 1122 \\
\hline 2009 & 28979 & 24937 & 3850 & 87 & & 106 & 21362 & 7618 & 18422 & 6515 & 2887 & 963 & 1138 \\
\hline \multicolumn{14}{|l|}{ Men } \\
\hline & MGSA & MGRO & MGRR & MGRU & MG & $\mathrm{GRX}$ & YCBF & YCBI & YCBL & YCBO & YCBR & YCBU & YCBX \\
\hline 2002 & 15099 & 12559 & 2444 & 33 & & 63 & 13606 & 1493 & 11430 & 1129 & 2131 & 313 & 480 \\
\hline 2003 & 15262 & 12566 & 2604 & 34 & & 58 & 13691 & 1570 & 11388 & 1178 & 2259 & 345 & 461 \\
\hline 2004 & 15405 & 12634 & 2659 & 39 & & 73 & 13776 & 1629 & 11413 & 1222 & 2313 & 346 & 456 \\
\hline 2005 & 15535 & 12768 & 2666 & 35 & & 67 & 13887 & 1649 & 11517 & 1251 & 2322 & 344 & 455 \\
\hline 2006 & 15662 & 12857 & 2710 & 38 & & 58 & 13975 & 1688 & 11579 & 1279 & 2355 & 356 & 452 \\
\hline 2007 & 15813 & 12950 & 2762 & 39 & & 61 & 14068 & 1745 & 11650 & 1301 & 2379 & 384 & 454 \\
\hline 2008 & 15894 & 13011 & 2780 & 37 & & 67 & 14072 & 1822 & 11643 & 1368 & 2393 & 387 & 461 \\
\hline 2009 & 15497 & 12657 & 2747 & 34 & & 59 & 13635 & 1862 & 11253 & 1404 & 2351 & 396 & 488 \\
\hline \multicolumn{14}{|c|}{ Women } \\
\hline & MGSB & MGRP & MGRS & MGRV & MG & GRY & YCBG & YCBJ & YCBM & YCBP & YCBS & YCBV & YCBY \\
\hline 2002 & 12823 & 11827 & 893 & 63 & & 40 & 7203 & 5620 & 6743 & 5084 & 434 & 459 & 670 \\
\hline 2003 & 12925 & 11861 & 961 & 60 & & 42 & 7224 & 5701 & 6729 & 5133 & 471 & 490 & 656 \\
\hline 2004 & 13080 & 12011 & 960 & 58 & & 51 & 7355 & 5726 & 6852 & 5159 & 476 & 484 & 617 \\
\hline 2005 & 13239 & 12161 & 970 & 61 & & 48 & 7598 & 5642 & 7079 & 5082 & 489 & 481 & 603 \\
\hline 2006 & 13367 & 12241 & 1027 & 59 & & 39 & 7667 & 5700 & 7133 & 5108 & 510 & 518 & 602 \\
\hline \multirow[t]{3}{*}{2009} & 13482 & 12280 & 1103 & 53 & & 46 & 7726 & 5756 & 7168 & 5111 & 536 & 567 & 650 \\
\hline & \multicolumn{8}{|c|}{ Temporary employees (reason for temporary working) } & \multicolumn{5}{|c|}{ Part-time workers (reasons for working part-time) ${ }^{2}$} \\
\hline & Total e & $\begin{array}{r}\text { Total as \% } \\
\text { of all pe } \\
\text { employees }\end{array}$ & $\begin{array}{r}\text { Could not } \\
\text { find } \\
\text { job }\end{array}$ & $\begin{array}{r}\% \text { that } \\
\text { could not } \\
\text { find } \\
\text { ermanent } \\
\text { job }\end{array}$ & $\begin{array}{r}\text { Did not } \\
\text { want a } \\
\text { permanent } \\
\text { job }\end{array}$ & $\begin{array}{r}\text { } \\
\text { cor } \\
\text { peri } \\
\text { tra }\end{array}$ & $\begin{array}{l}\text { ad a } \\
\text { tract } \\
\text { with } \\
\text { d of Som } \\
\text { ning }\end{array}$ & $\begin{array}{l}\text { ther } \\
\text { son }\end{array}$ & $\begin{array}{l}\text { Coulc } \\
\text { full- }\end{array}$ & $\begin{array}{l}\% \\
\text { ot } \\
\text { id } \\
\text { e could } \\
\text { b }\end{array}$ & $\begin{array}{lr}\text { t } & \\
\text { d } & \text { Did no } \\
\text { d } & \text { want } \\
\text { e } & \text { full time } \\
b & \text { jol }\end{array}$ & $\begin{array}{r}\text { III or } \\
\text { disabled }\end{array}$ & $\begin{array}{l}\text { Student or } \\
\text { at school }\end{array}$ \\
\hline
\end{tabular}

\begin{tabular}{|c|c|c|c|c|c|c|c|c|c|c|c|c|c|}
\hline \\
\hline $\begin{array}{l}2002 \\
2003 \\
2004 \\
2005 \\
2006\end{array}$ & $\begin{array}{l}\text { YCBZ } \\
1587 \\
1516 \\
1509 \\
1445 \\
1479\end{array}$ & $\begin{array}{r}\text { YCCC } \\
6.5 \\
6.2 \\
6.1 \\
5.8 \\
5.9\end{array}$ & $\begin{array}{r}\text { YCCF } \\
423 \\
399 \\
384 \\
362 \\
374\end{array}$ & $\begin{array}{r}\text { YCCI } \\
26.7 \\
26.3 \\
25.5 \\
25.0 \\
25.3\end{array}$ & $\begin{array}{r}\text { YCCL } \\
467 \\
455 \\
430 \\
389 \\
427\end{array}$ & $\begin{array}{r}\text { YCCO } \\
82 \\
87 \\
99 \\
100 \\
103\end{array}$ & $\begin{array}{r}\text { YCCR } \\
613 \\
577 \\
597 \\
593 \\
576\end{array}$ & $\begin{array}{l}\text { YCCU } \\
6985 \\
7145 \\
7211 \\
7157 \\
7260\end{array}$ & $\begin{array}{r}\text { YCCX } \\
571 \\
567 \\
549 \\
589 \\
637\end{array}$ & $\begin{array}{r}\text { YCDA } \\
8.2 \\
8.0 \\
7.6 \\
8.2 \\
8.8\end{array}$ & $\begin{array}{l}\text { YCDD } \\
5148 \\
5255 \\
5291 \\
5223 \\
5221\end{array}$ & $\begin{array}{r}\text { YCDG } \\
138 \\
156 \\
177 \\
167 \\
181\end{array}$ & $\begin{array}{ll}\text { YCDJ } \\
1 & 100 \\
1 & 134 \\
1 & 163 \\
1 & 147 \\
1 & 181\end{array}$ \\
\hline $\begin{array}{l}2007 \\
2008 \\
2009\end{array}$ & $\begin{array}{l}1500 \\
1399 \\
1430\end{array}$ & $\begin{array}{l}5.9 \\
5.5 \\
5.7\end{array}$ & $\begin{array}{l}396 \\
360 \\
452\end{array}$ & $\begin{array}{l}26.4 \\
25.7 \\
31.6\end{array}$ & $\begin{array}{l}426 \\
404 \\
376\end{array}$ & $\begin{array}{l}94 \\
85 \\
82\end{array}$ & $\begin{array}{l}583 \\
550 \\
520\end{array}$ & $\begin{array}{l}7275 \\
7353 \\
7448\end{array}$ & $\begin{array}{l}689 \\
713 \\
971\end{array}$ & $\begin{array}{r}9.5 \\
9.7 \\
12.9\end{array}$ & $\begin{array}{ll}5 & 226 \\
5 & 247 \\
5 & 163\end{array}$ & $\begin{array}{l}175 \\
197 \\
186\end{array}$ & $\begin{array}{ll}1 & 152 \\
1 & 153 \\
1 & 114\end{array}$ \\
\hline $\begin{array}{l}\text { Men } \\
2002 \\
2003 \\
2004 \\
2005 \\
2006\end{array}$ & $\begin{array}{r}\text { YCCA } \\
721 \\
697 \\
709 \\
681 \\
677\end{array}$ & $\begin{array}{r}\text { YCCD } \\
5.8 \\
5.5 \\
5.6 \\
5.3 \\
5.3\end{array}$ & $\begin{array}{r}\text { YCCG } \\
233 \\
225 \\
222 \\
204 \\
196\end{array}$ & $\begin{array}{r}\text { YCCJ } \\
32.3 \\
32.2 \\
31.3 \\
30.0 \\
29.0\end{array}$ & $\begin{array}{r}\text { YCCM } \\
186 \\
185 \\
175 \\
169 \\
176\end{array}$ & $\begin{array}{r}\text { YCCP } \\
43 \\
38 \\
47 \\
53 \\
53\end{array}$ & $\begin{array}{r}\text { YCCS } \\
260 \\
250 \\
265 \\
256 \\
252\end{array}$ & $\begin{array}{r}\text { YCCV } \\
1442 \\
1523 \\
1567 \\
1595 \\
1634\end{array}$ & $\begin{array}{r}\text { YCCY } \\
233 \\
249 \\
246 \\
236 \\
263\end{array}$ & $\begin{array}{r}\text { YCDB } \\
16.2 \\
16.4 \\
15.7 \\
14.8 \\
16.1\end{array}$ & $\begin{array}{r}\text { YCDE } \\
650 \\
705 \\
745 \\
769 \\
775\end{array}$ & $\begin{array}{r}\text { YCDH } \\
60 \\
69 \\
69 \\
74 \\
75\end{array}$ & $\begin{array}{r}\text { YCDK } \\
489 \\
489 \\
498 \\
504 \\
511\end{array}$ \\
\hline $\begin{array}{l}2007 \\
2008 \\
2009\end{array}$ & $\begin{array}{l}699 \\
641 \\
677\end{array}$ & $\begin{array}{l}5.4 \\
4.9 \\
5.3\end{array}$ & $\begin{array}{l}211 \\
184 \\
243\end{array}$ & $\begin{array}{l}30.1 \\
28.7 \\
36.0\end{array}$ & $\begin{array}{l}186 \\
165 \\
152\end{array}$ & $\begin{array}{l}45 \\
44 \\
43\end{array}$ & $\begin{array}{l}258 \\
249 \\
240\end{array}$ & $\begin{array}{l}1684 \\
1755 \\
1800\end{array}$ & $\begin{array}{l}283 \\
306 \\
432\end{array}$ & $\begin{array}{l}16.9 \\
17.5 \\
24.0\end{array}$ & $\begin{array}{l}820 \\
854 \\
795\end{array}$ & $\begin{array}{l}75 \\
76 \\
78\end{array}$ & $\begin{array}{l}495 \\
503 \\
478\end{array}$ \\
\hline $\begin{array}{l}\text { Wome } \\
2002 \\
2003 \\
2004 \\
2005 \\
2006\end{array}$ & $\begin{array}{r}\text { YCCB } \\
866 \\
819 \\
800 \\
763 \\
802\end{array}$ & $\begin{array}{r}\text { YCCE } \\
7.3 \\
6.9 \\
6.7 \\
6.3 \\
6.6\end{array}$ & $\begin{array}{r}\text { YCCH } \\
191 \\
174 \\
162 \\
158 \\
178\end{array}$ & $\begin{array}{r}\text { YCCK } \\
22.0 \\
21.2 \\
20.3 \\
20.7 \\
22.1\end{array}$ & $\begin{array}{r}\text { YCCN } \\
282 \\
270 \\
254 \\
220 \\
251\end{array}$ & $\begin{array}{r}Y C C Q \\
40 \\
49 \\
51 \\
48 \\
50\end{array}$ & $\begin{array}{r}\text { YCCT } \\
354 \\
327 \\
333 \\
338 \\
324\end{array}$ & $\begin{array}{r}\text { YCCW } \\
5544 \\
5622 \\
5644 \\
5563 \\
5626\end{array}$ & $\begin{array}{r}\text { YCCZ } \\
337 \\
318 \\
303 \\
353 \\
375\end{array}$ & $\begin{array}{r}\text { YCDC } \\
6.1 \\
5.6 \\
5.3 \\
6.4 \\
6.7\end{array}$ & $\begin{array}{l}\text { YCDF } \\
4498 \\
4550 \\
4546 \\
4454 \\
4447\end{array}$ & $\begin{array}{r}\text { YCDI } \\
78 \\
87 \\
108 \\
93 \\
107\end{array}$ & $\begin{array}{r}\text { YCDL } \\
611 \\
645 \\
666 \\
643 \\
671\end{array}$ \\
\hline $\begin{array}{l}2007 \\
2008 \\
2009\end{array}$ & $\begin{array}{l}801 \\
758 \\
753\end{array}$ & $\begin{array}{l}6.5 \\
6.1 \\
6.1\end{array}$ & $\begin{array}{l}186 \\
176 \\
209\end{array}$ & $\begin{array}{l}23.2 \\
23.2 \\
27.8\end{array}$ & $\begin{array}{l}241 \\
240 \\
224\end{array}$ & $\begin{array}{l}49 \\
41 \\
40\end{array}$ & $\begin{array}{l}325 \\
301 \\
280\end{array}$ & $\begin{array}{l}5591 \\
5598 \\
5678\end{array}$ & $\begin{array}{l}405 \\
407 \\
540\end{array}$ & $\begin{array}{l}7.3 \\
7.3 \\
9.5\end{array}$ & $\begin{array}{l}4406 \\
4393 \\
4368\end{array}$ & $\begin{array}{l}100 \\
121 \\
108\end{array}$ & $\begin{array}{l}657 \\
650 \\
637\end{array}$ \\
\hline
\end{tabular}

1 The split between full-time and part-time employment is based on repon- 2 These series cover employees and self-employed only. 
Labour Market

All aged 16 and

over

$16-59 / 64$

16-17

$18-24$

25-34

$35-49$

$65+(m)$

In Employment

People

2003

2004

2005

2006
2007

2008

2009

MGRZ

28186

28485

28774

29030

29222

YBSE
27240
27486
27705
27863
27996

$\begin{array}{rr}\text { YBTO } & \text { YBTR } \\ 652 & 3415 \\ 642 & 3528 \\ 607 & 3539 \\ 559 & 3618 \\ 534 & 3657 \\ 525 & 3663 \\ 430 & 3448\end{array}$

YBTU
6362
6283
6297
6261
6254
6285
6222

YBTX
10591
10742
10885
10975
11039
11065
10916

MGUW

$$
6221
$$

6293

6379

6451

29443

28120

28979

27597

MGSA

15262

15405

YBSF

2003

2004
2005

2006

2007

15662

14932

15068

15175

15272

\section{YBTP}

316
309

309
293

260

1802
1868

1879

1912

1943

YBTV

3473
3417

3432

3423

3443

1935

15452
15055

260

3444

3404

6513

6583

6580

2009

15497

15055

Women

2003

MGSB

12925

YBSG

12309

$13080 \quad 12419$

$13239 \quad 12530$

13367

12591

13409

12589

13549

12669

2008

13482

12542

YBTQ

336
333

333

315

299
277

1788

YBTY

5659

5743

5781

5845

MGUX

3682

3731

3790

3832

3885

5871

3943

3922

443

YBTT YBTW

$1612 \quad 2889$

2866

1660

2865

2838

1706
1714

2812

5744

MGUY

YBTZ
4932

4998

5104

5130

5160

2539
2562

2589

2619

2627

1729

2841

5195

2640

2658

MGUZ

234

1661

2818

5171

998
1070
1167

1226

1323

1383

Employment rates(\%)

People

2003

2004

2005

2006

2007

2008

2009

MGSR

MGSU

YBUA

YBUD

YBUG

$\begin{array}{ll}74.6 & 42.7 \\ 74.7 & 41.2\end{array}$

74.7
74.7

41.2

38.6

35.5
33.7

66.5
66.9
65.6

65.6

65.5
64.8

79.5
79.7

80.2

80.1

80.3

YBUJ

82.1
82.2

82.4

82.3

82.3

80.3
78.3

82.4

YBUM

69.6
70.0

70.0
70.4

70.4

71.3

MGVA

330
337

361

390

407

33.1

63.8
59.5

71.9

71.3

MGVB

616

616
661
709

709

776
820

880

940

Men

2003

2004

2005

2006
2007

58.6

72.8

YBUB

MGSS

MGSV

$\begin{array}{ll}67.0 & 79.2 \\ 67.1 & 79.3\end{array}$

79.3

38.7

36.3

32.3

YBUE

69.8

YBUH

YBUK

YBUN

BUN
71.6
72.0

72.0

72.4

72.3

72.6

YBUP

$\begin{array}{lll}68.8 & 88.1 & 88.5\end{array}$

$\begin{array}{lll}67.6 & 88.1 & 88.7 \\ 68.7 & 88.8\end{array}$

73.0

71.9

8.9
9.4

9.4
9.9

10.7

11.0

66.8

78.5

31.9

65.9

87.9

88.6

60.7

85.2

86.9

11.7

12.0

Women

2003

2004
2005

2005

2007

MGST
53.1
53.5
53.7
53.8
53.7

53.8
53.1

MGSW YBUC

$69.7 \quad$ YBUC

$\begin{array}{ll}69.7 & 45.1 \\ 69.9 & 43.9\end{array}$

$70.0 \quad 41.0$

$\begin{array}{ll}70.0 & 39.0\end{array}$

YBUF

YBUF YBUI

$63.1 \quad 71.6$

63.7

62.3
62.8

62.0

72.1
72.3

72.2

72.0

YBUL

75.6

75.6
76.4

76.4
76.1

75.9

61.5
58.2

72.8
71.4

76.3
76.2

Sources: Labour Force Survey, Office for National Statistics;

1 See chapter text.

Denominator $=$ all persons in the relevant age group

$\begin{array}{ll}70.2 & 34.3 \\ 69.2 & 30.8\end{array}$

34.3
30.8

58.2

Helpline: 01633456901 
At mid-June each year. Seasonally adjusted

Thousands

\begin{tabular}{|c|c|c|c|c|c|c|c|c|c|c|c|c|}
\hline & & 1999 & 2000 & 2001 & 2002 & 2003 & 2004 & 2005 & 2006 & 2007 & 2008 & 2009 \\
\hline \multicolumn{13}{|l|}{ United Kingdom } \\
\hline Claimant count & BCJD & 1248.1 & 1088.4 & 969.9 & 946.6 & 933.0 & 853.3 & 861.8 & 945.0 & 863.6 & 905.1 & 1531.8 \\
\hline Males & DPAE & 955.0 & 831.6 & 739.6 & 717.1 & 700.3 & 636.2 & 639.7 & 697.5 & 630.9 & 665.1 & 1127.8 \\
\hline Females & DPAF & 293.1 & 256.8 & 230.3 & 229.6 & 232.8 & 217.1 & 222.1 & 247.5 & 232.8 & 240.1 & 404.0 \\
\hline Workforce jobs & DYDC & 29127 & 29554 & 29890 & 30064 & 30350 & 30671 & 31012 & 31257 & 31471 & 31661 & 30987 \\
\hline Males & KAMS & 15663 & 15772 & 15992 & 16002 & 16245 & 16376 & 16487 & 16662 & 16773 & 16908 & 16405 \\
\hline Females & KAMT & 13464 & 13782 & 13898 & 14061 & 14105 & 14296 & 14525 & 14596 & 14698 & 14753 & 14582 \\
\hline Males & KAMV & 201 & 199 & 196 & 197 & 203 & 199 & 191 & 185 & 180 & 176 & 179 \\
\hline Females & KAMW & 17 & 18 & 18 & 18 & 19 & 19 & 18 & 18 & 18 & 18 & 18 \\
\hline Self-employment jobs & DYZN & 3688 & 3579 & 3604 & 3674 & 3883 & 3964 & 3943 & 4056 & 4169 & 4181 & 4222 \\
\hline Males & KAMZ & 2642 & 2552 & 2593 & 2637 & 2785 & 2863 & 2840 & 2879 & 2953 & 2961 & 2953 \\
\hline Females & KANA & 1046 & 1027 & 1012 & 1037 & 1097 & 1101 & 1103 & 1177 & 1216 & 1220 & 1269 \\
\hline Employees jobs & BCAJ & 25091 & 25639 & 25973 & 26085 & 26152 & 26381 & 26763 & 26933 & 27051 & 27232 & 26522 \\
\hline Males & KANC & 12740 & 12948 & 13142 & 13114 & 13201 & 13249 & 13398 & 13559 & 13609 & 13739 & 13248 \\
\hline Females & KAND & 12351 & 12691 & 12831 & 12971 & 12951 & 13132 & 13365 & 13374 & 13442 & 13493 & 13274 \\
\hline $\begin{array}{l}\text { of whom } \\
\text { Total, production and }\end{array}$ & & & & & & & & & & & & \\
\hline Total, all manufacturing & & & & & & & & & & & & \\
\hline industries & KANG & 4059 & 3959 & 3805 & 3599 & 3410 & 3246 & 3102 & 2975 & 2911 & 2867 & 2645 \\
\hline Government-supported trainees & KANH & 131 & 119 & 99 & 90 & 92 & 108 & 96 & 65 & 53 & 54 & 46 \\
\hline Males & KANI & 81 & 73 & 62 & 55 & 55 & 65 & 58 & 38 & 31 & 32 & 25 \\
\hline Females & KANJ & 50 & 46 & 38 & 36 & 37 & 44 & 38 & 27 & 21 & 22 & 21 \\
\hline \multicolumn{13}{|l|}{ Great Britain } \\
\hline Claimant count & DPAG & 1197.3 & 1046.3 & 930.5 & 910.2 & 898.5 & 822.5 & 833.2 & 917.1 & 839.3 & 877.3 & 1482.7 \\
\hline Males & ZSDP & 915.7 & 799.6 & 709.7 & 689.3 & 673.9 & 612.8 & 618.0 & 676.6 & 612.5 & 643.5 & 1089.3 \\
\hline Females & ZSDQ & 281.6 & 246.8 & 220.8 & 220.9 & 224.6 & 209.8 & 215.1 & 240.5 & 226.8 & 233.8 & 393.4 \\
\hline Workforce jobs & KANQ & 28394 & 28804 & 29127 & 29289 & 29561 & 29870 & 30186 & 30423 & 30632 & 30810 & 30152 \\
\hline Males & KANR & 15267 & 15366 & 15580 & 15588 & 15822 & 15946 & 16044 & 16214 & 16327 & 16454 & 15964 \\
\hline Females & KANS & 13126 & 13438 & 13547 & 13702 & 13739 & 13924 & 14142 & 14209 & 14305 & 14355 & 14188 \\
\hline HM Forces & $\mathrm{BCAH}$ & 218 & 217 & 214 & 214 & 223 & 218 & 210 & 204 & 198 & 193 & 197 \\
\hline Males & KANU & 201 & 199 & 196 & 197 & 203 & 199 & 191 & 185 & 180 & 176 & 179 \\
\hline Females & KANV & 17 & 18 & 18 & 18 & 19 & 19 & 18 & 18 & 18 & 18 & 18 \\
\hline Self-employment jobs & KANW & 3592 & 3480 & 3500 & 3572 & 3774 & 3851 & 3820 & 3933 & 4055 & 4059 & 4100 \\
\hline Employee jobs & KANZ & 24465 & 24997 & 25321 & 25419 & 25478 & 25699 & 26067 & 26227 & 26332 & 26507 & 25813 \\
\hline Males & KAOA & 12429 & 12630 & 12821 & 12788 & 12873 & 12917 & 13062 & 13215 & 13258 & 13386 & 12908 \\
\hline Females & KAOB & 12036 & 12367 & 12500 & 12631 & 12605 & 12782 & 13005 & 13012 & 13074 & 13120 & 12905 \\
\hline \multirow{2}{*}{\multicolumn{13}{|c|}{$\begin{array}{l}\text { of whom } \\
\text { Total, production and }\end{array}$}} \\
\hline & & & & & & & & & & & & \\
\hline construction industries & KAOC & 5239 & 5205 & 5052 & 4813 & 4616 & 4464 & 4344 & 4290 & 4239 & 4242 & 3991 \\
\hline \multicolumn{13}{|l|}{ Total, all manufacturing } \\
\hline industries & KAOD & 3954 & 3856 & 3704 & 3501 & 3318 & 3157 & 3015 & 2888 & 2823 & 2778 & 2564 \\
\hline Government-supported trainees & KAOE & 120 & 110 & 92 & 84 & 86 & 102 & 90 & 58 & 46 & 51 & 42 \\
\hline Males & KAOF & 73 & 67 & 57 & 50 & 51 & 60 & 53 & 33 & 27 & 29 & 22 \\
\hline Females & KAOG & 47 & 43 & 35 & 34 & 35 & 42 & 36 & 25 & 19 & 21 & 20 \\
\hline
\end{tabular}

Note. Because the figures have been rounded independently totals may differ from the sum of the components. Also the totals may include some

Sources: Business Statistics Division, Office for National Statistics; employees whose industrial classification could not be ascertained.

1 The data in this table have not been adjusted to reflect the 2001 Census population data. See chapter text.

2 There is a discontinuity in the employee jobs series between December 2005 and September 2006 due to improvements to the annual benchmark.

Further information can be found at:

http://www.statistics.gov.uk/statbase/product.asp?vlnk=9765 


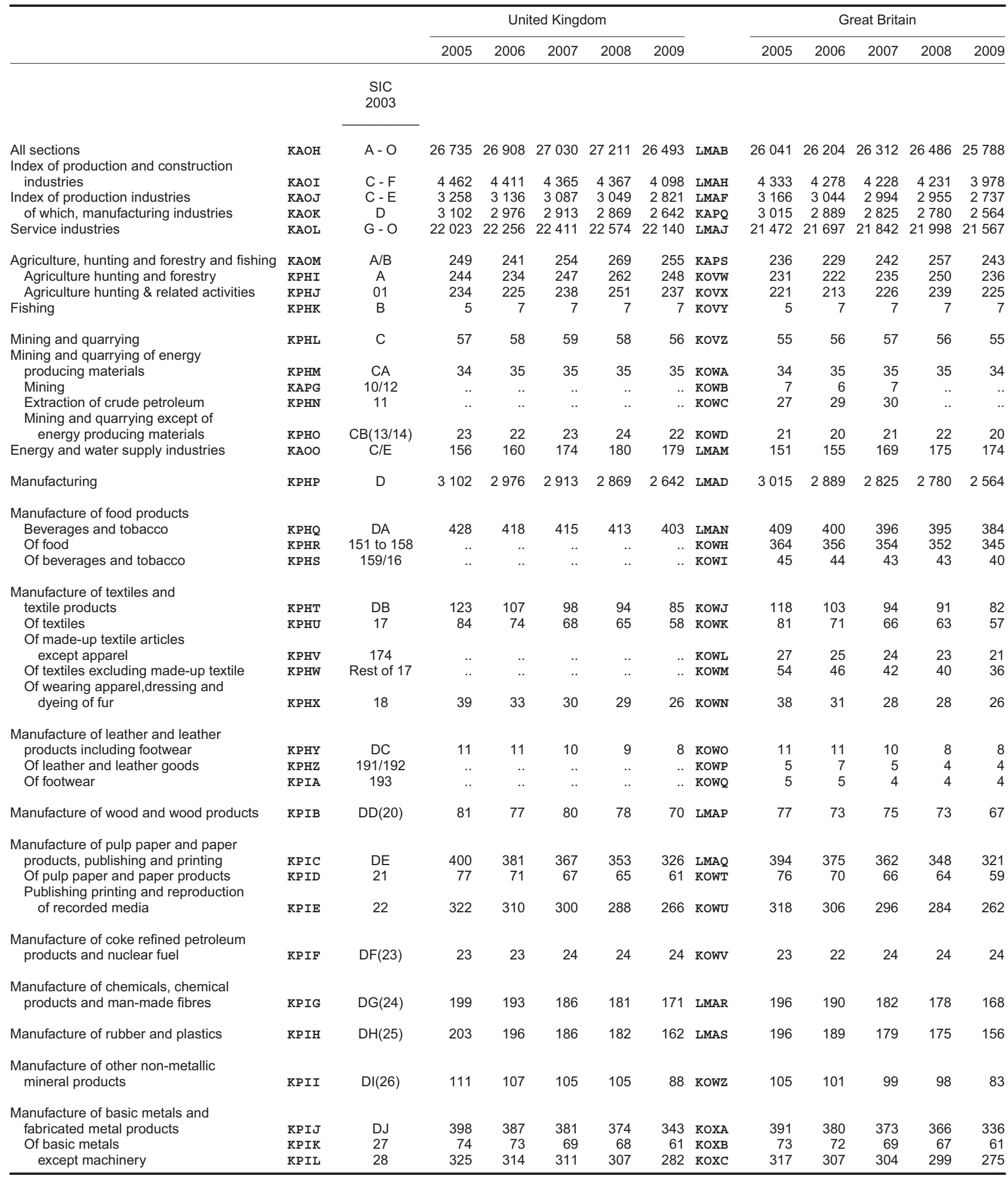




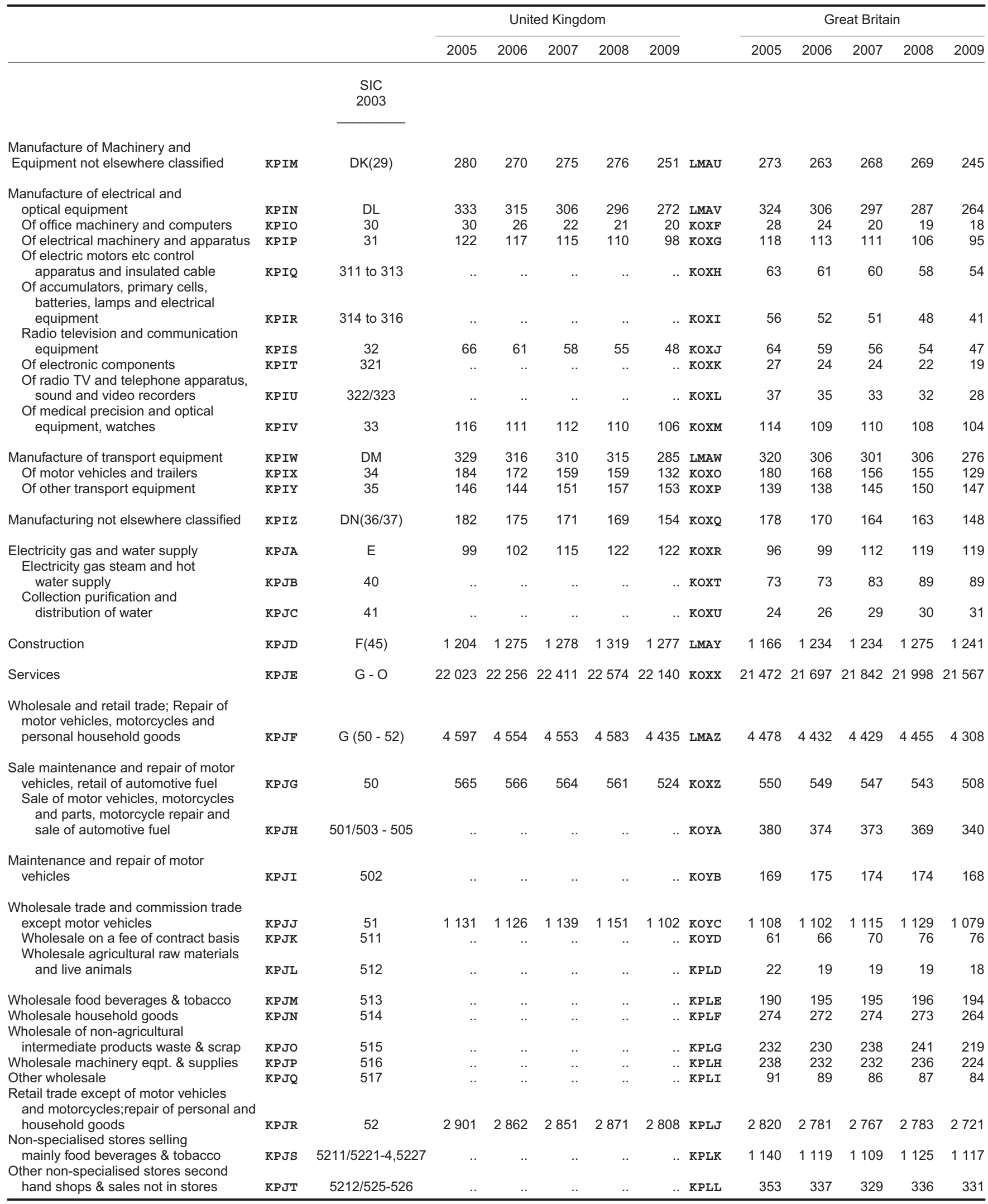




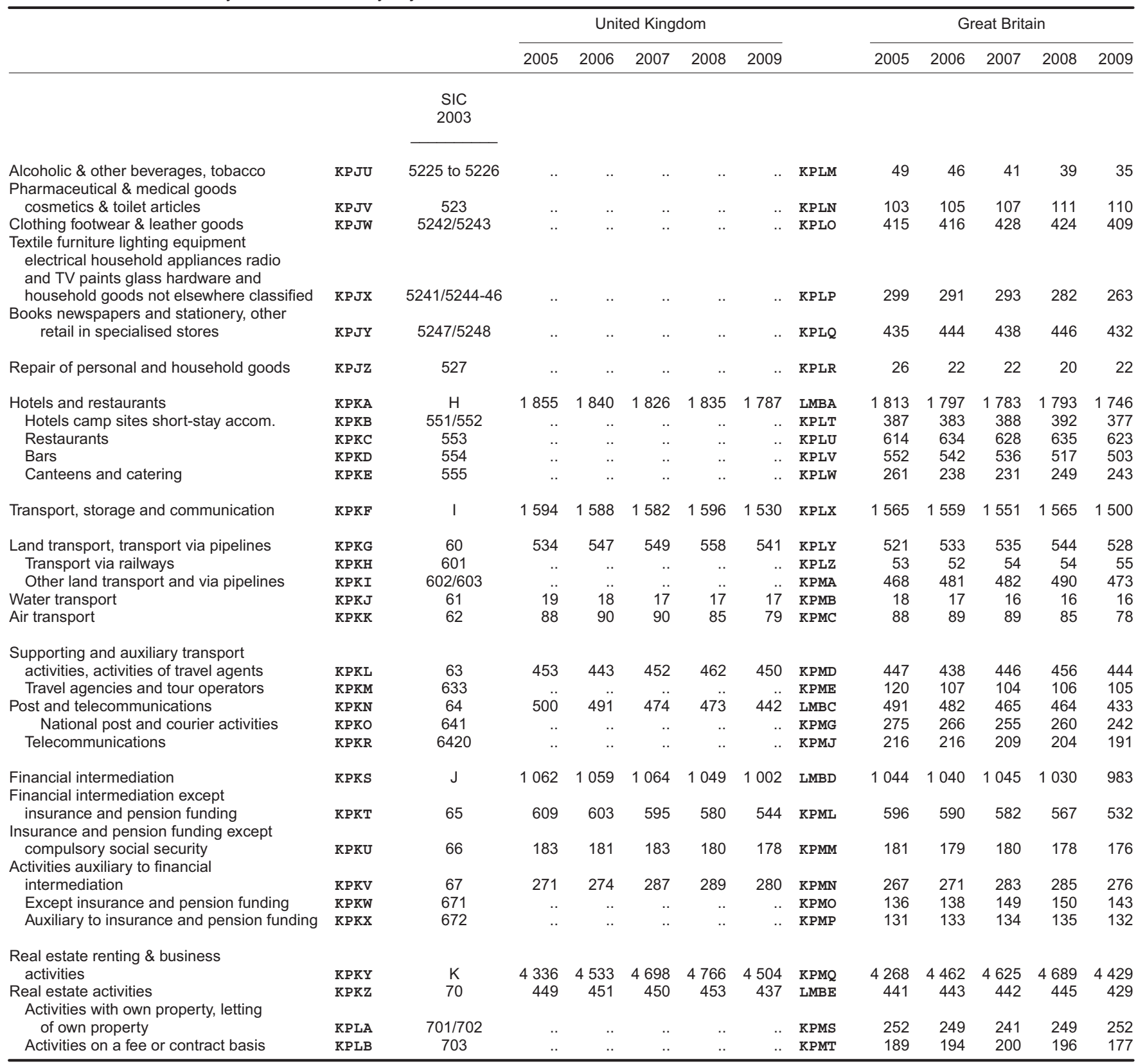




\section{Labour Market}

75 Employee jobs: by industry ${ }^{1,2,3}$

continued

Standard Industrial Classification 2003

At June each year. Not seasonally adjusted

Thousand

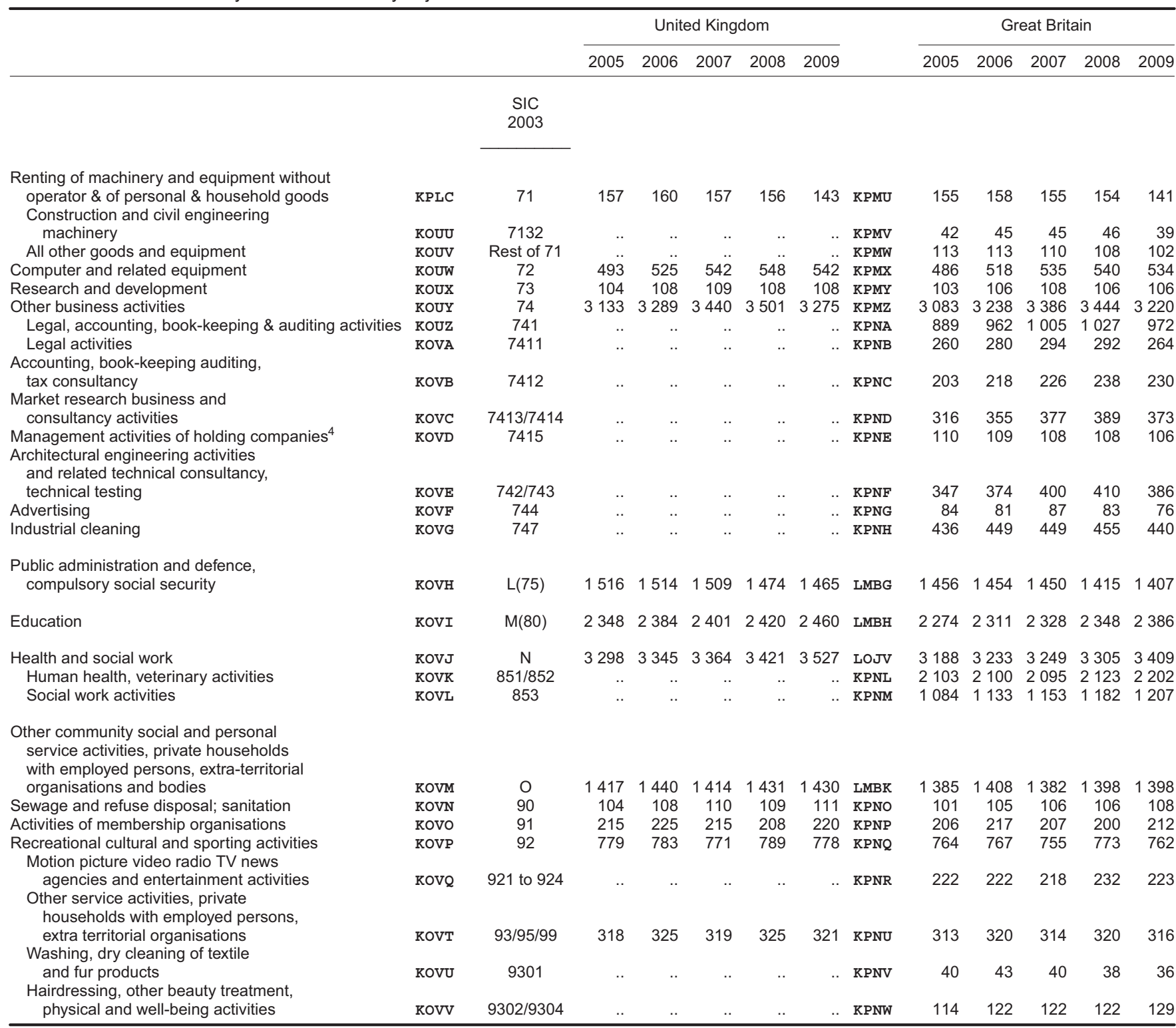

Note. Because the figures have been rounded independently totals may

Sources: Department of Manpower Services (Northern Ireland); differ from the sum of the components. Also the totals may include some Business Statistics Division, ONS: 01633456776 employees whose industrial classification could not be ascertained.

1 See chapter text. The data in this table have not been adjusted to reflect the 2001 Census population data.

2 All figures have been revised. For further information see: http://www. statistics.gov.uk/cci/article. asp? $\mathrm{id}=1340$

3 There is a discontinuity in the employee jobs series between December 2005 and September 2006 due to improvements to the annual benchmark. Further information can be found at: http://www.statistics.gov.uk/Statbase/Product.asp?vlnk=9765 


\begin{tabular}{|c|c|c|c|c|c|}
\hline & \multirow{2}{*}{$\begin{array}{l}\text { Total weekly hours } \\
(\text { (millions })^{1,2}\end{array}$} & \multicolumn{4}{|c|}{ Average(mean) actual weekly hours worked } \\
\hline & & All workers ${ }^{1}$ & Full-time workers ${ }^{3}$ & Part-time workers ${ }^{3}$ & Second jobs \\
\hline \multicolumn{6}{|c|}{ People } \\
\hline & YBUS & YBUV & YBUY & YBVB & YBVE \\
\hline 1999 & 892.7 & 32.9 & 38.2 & 15.3 & 9.1 \\
\hline 2000 & 894.8 & 32.6 & 37.9 & 15.5 & 9.1 \\
\hline 2001 & 903.5 & 32.7 & 37.9 & 15.6 & 9.4 \\
\hline 2002 & 901.0 & 32.3 & 37.5 & 15.6 & 9.4 \\
\hline 2003 & 904.6 & 32.2 & 37.4 & 15.6 & 9.3 \\
\hline 2004 & 912.1 & 32.1 & 37.3 & 15.6 & 9.3 \\
\hline 2005 & 923.7 & 32.2 & 37.3 & 15.7 & 9.5 \\
\hline 2006 & 928.5 & 32.0 & 37.1 & 15.6 & 9.4 \\
\hline 2007 & 936.1 & 32.1 & 37.2 & 15.6 & 9.5 \\
\hline 2008 & 940.7 & 32.0 & 37.1 & 15.6 & 9.7 \\
\hline 2009 & 913.3 & 31.6 & 36.8 & 15.5 & 9.5 \\
\hline \multicolumn{6}{|l|}{ Men } \\
\hline & YBUT & YBUW & YBUZ & YBVC & YBVF \\
\hline 1999 & 564.0 & 38.3 & 40.2 & 15.1 & 9.8 \\
\hline 2000 & 564.2 & 37.9 & 39.8 & 15.3 & 9.9 \\
\hline 2001 & 567.0 & 37.8 & 39.7 & 15.3 & 10.3 \\
\hline 2002 & 561.9 & 37.3 & 39.3 & 15.1 & 10.3 \\
\hline 2003 & 564.3 & 37.0 & 39.2 & 15.3 & 10.1 \\
\hline 2004 & 569.0 & 37.0 & 39.2 & 15.6 & 10.3 \\
\hline 2005 & 572.2 & 36.9 & 39.1 & 15.6 & 10.3 \\
\hline 2006 & 573.6 & 36.7 & 38.9 & 15.5 & 10.1 \\
\hline 2007 & 580.2 & 36.8 & 39.0 & 15.5 & 10.6 \\
\hline 2008 & 580.8 & 36.6 & 39.0 & 15.4 & 10.8 \\
\hline 2009 & 559.0 & 36.1 & 38.5 & 15.5 & 10.4 \\
\hline \multicolumn{6}{|c|}{ Women } \\
\hline & YBUU & YBUX & YBVA & YBVD & YBVG \\
\hline 1999 & 328.6 & 26.5 & 34.5 & 15.4 & 8.6 \\
\hline 2000 & 330.6 & 26.3 & 34.2 & 15.5 & 8.5 \\
\hline 2001 & 336.5 & 26.5 & 34.3 & 15.7 & 8.8 \\
\hline 2004 & 343.1 & 26.3 & 33.9 & 15.6 & 8.5 \\
\hline 2005 & 351.5 & 26.6 & 34.0 & 15.8 & 8.9 \\
\hline 2006 & 354.9 & 26.6 & 34.0 & 15.7 & 8.9 \\
\hline 2007 & 355.9 & 26.6 & 33.9 & 15.6 & 8.8 \\
\hline 2008 & 359.9 & 26.6 & 33.8 & 15.6 & 8.9 \\
\hline 2009 & 354.2 & 26.3 & 33.7 & 15.4 & 8.8 \\
\hline
\end{tabular}

See chapter text.

Sources: Labour Force Survey, Office for National Statistics;

1 Main and second job Helpline: 01633456901

actual weekly hours worked including paid and unpaid overtime.

3 Main job only. The split between full-time and part-time employment is

based on respondents' self-classification. 


\begin{tabular}{|c|c|c|c|c|c|c|c|c|c|c|c|c|c|c|c|}
\hline & & $\begin{array}{r}2006 \\
\text { Q4 }\end{array}$ & 20 & 7 & $\begin{array}{r}007 \\
\text { Q2 }\end{array}$ & $\begin{array}{r}2007 \\
\text { Q3 }\end{array}$ & $\begin{array}{r}2007 \\
\text { Q4 }\end{array}$ & $\begin{array}{r}2008 \\
\text { Q1 }\end{array}$ & $\begin{array}{r}2008 \\
\text { Q2 }\end{array}$ & $\begin{array}{r}200 \\
Q\end{array}$ & & $\begin{array}{l}08 \\
Q 4\end{array}$ & $\begin{array}{r}2009 \\
\text { Q1 }\end{array}$ & $\begin{array}{r}2009 \\
\text { Q2 }\end{array}$ & $\begin{array}{r}2009 \\
\text { Q3 }\end{array}$ \\
\hline \multicolumn{16}{|c|}{ EUROSTAT Employment rates } \\
\hline Austria & YXSN & 70.6 & 70 & & 1.5 & 72.5 & 71.3 & 71.0 & 72.3 & \multicolumn{2}{|c|}{72.8} & 72.2 & 70.8 & 71.7 & 72.3 \\
\hline Belgium & YXSO & 62.1 & 61 & & 1.6 & 62.1 & 62.7 & 62.6 & 62.0 & 62.6 & \multicolumn{2}{|c|}{62.4} & 61.7 & 61.5 & 61.4 \\
\hline Bulgaria & A495 & 59.8 & 59 & & 1.6 & 62.7 & 62.9 & 62.6 & 63.9 & 65. & & 4.3 & 62.6 & 63.3 & 63.1 \\
\hline Cyprus & $\mathrm{A} 4 \mathrm{AC}$ & 70.4 & 69 & & 1.2 & 71.3 & 71.5 & 70.2 & 71.1 & 71. & & 1.1 & 69.5 & 70.2 & 70.0 \\
\hline Czech Republic & $\mathrm{A} 4 \mathrm{AD}$ & 65.6 & 65 & & 6.0 & 66.3 & 66.5 & 66.1 & 66.6 & 66.7 & & 6.8 & 65.6 & 65.4 & 65.2 \\
\hline Denmark & YXSP & 77.9 & 76 & & 7.3 & 77.1 & 77.4 & 77.0 & 78.4 & 78. & & 8.3 & 76.2 & 76.2 & 76.3 \\
\hline Estonia & $\mathrm{A} 4 \mathrm{AE}$ & 68.1 & 68 & & 9.7 & 70.2 & 69.1 & 69.5 & 69.8 & 70. & & 9.6 & 65.3 & 63.8 & 63.4 \\
\hline France & YXSR & 63.7 & 63 & & 4.4 & 64.9 & 64.5 & 64.6 & 65.1 & 65. & & 4.7 & 64.1 & 64.6 & 64.6 \\
\hline Germany & YXSS & 68.3 & 68 & & 9.1 & 69.9 & 70.0 & 70.0 & 70.3 & 71.3 & & 1.3 & 70.4 & 70.8 & 71.0 \\
\hline Greece & YXST & 61.0 & 60 & & 1.5 & 61.8 & 61.5 & 61.3 & 62.2 & 62.2 & & 1.7 & 61.0 & 61.6 & 61.7 \\
\hline Hungary & $\mathrm{A} 4 \mathrm{AF}$ & 57.6 & 56 & & 7.6 & 57.7 & 57.1 & 56.1 & 56.5 & 57. & & 6.7 & 55.1 & 55.6 & 55.5 \\
\hline Ireland & YXSU & 68.7 & 68 & & 8.9 & 69.9 & 69.0 & 68.5 & 68.1 & 68. & & 5.6 & 62.8 & 62.2 & 61.8 \\
\hline Italy & YXSV & 58.5 & 57 & & 8.9 & 59.1 & 58.7 & 58.3 & 59.2 & 59. & & 8.5 & 57.4 & 57.9 & 57.5 \\
\hline Latvia & $\mathrm{A} 4 \mathrm{AG}$ & 67.4 & 66 & & 7.6 & 69.0 & 70.3 & 69.6 & 69.5 & 69. & & 6.5 & 64.3 & 61.4 & 59.8 \\
\hline Lithuania & $\mathrm{A} 4 \mathrm{AH}$ & 63.5 & 63 & & 5.4 & 66.1 & 64.4 & 63.9 & 64.6 & 65. & & 3.8 & 61.0 & 60.3 & 60.4 \\
\hline Luxembourg & YXSW & 63.6 & 63 & & 3.6 & 64.7 & 64.4 & 62.8 & 64.4 & 63. & & 2.6 & 64.5 & 65.7 & 65.8 \\
\hline Poland & $\mathrm{A} 4 \mathrm{AJ}$ & 55.7 & 55 & & 6.8 & 57.8 & 58.1 & 58.0 & 58.9 & 60. & & 0.0 & 58.9 & 59.3 & 59.9 \\
\hline Portugal & YXSY & 67.6 & 67 & & 7.6 & 68.1 & 68.1 & 68.1 & 68.6 & 68. & & 7.9 & 67.0 & 66.7 & 65.8 \\
\hline Romania & A494 & 57.4 & 57 & & 9.6 & 60.5 & 57.9 & 57.7 & 59.7 & 60 & & 8.3 & 57.4 & 59.2 & 60.4 \\
\hline Slovak Republic & $\mathrm{A} 4 \mathrm{AK}$ & 60.2 & 60 & & 0.4 & 60.7 & 61.6 & 61.3 & 61.7 & 63. & & 2.9 & 61.0 & 60.4 & 60.1 \\
\hline Slovenia & A4AL & 66.0 & 66 & & 8.3 & 69.0 & 67.7 & 67.1 & 68.3 & 70. & & 8.8 & 66.7 & 67.6 & 68.3 \\
\hline Spain & YXSZ & 65.2 & 65 & & 5.8 & 66.0 & 65.5 & 65.1 & 65.0 & 64. & & 2.8 & 60.4 & 59.9 & 59.7 \\
\hline Sweden & YXTA & 73.2 & 72 & & 4.3 & 75.7 & 74.0 & 73.4 & 74.8 & 75. & & 3.4 & 71.9 & 72.7 & 72.9 \\
\hline United Kingdom & ANZ 6 & 71.6 & 71 & & 1.2 & 71.6 & 71.9 & 71.6 & 71.6 & 71. & & 1.3 & 70.4 & 69.6 & 69.8 \\
\hline Total EU ${ }^{3}$ & A496 & 64.8 & 64 & & 5.4 & 66.0 & 65.7 & 65.5 & 66.0 & 66. & & 5.8 & 64.6 & 64.8 & 64.8 \\
\hline Eurozone $^{3}$ & YXTC & 65.0 & 64 & & 5.6 & 66.1 & 65.9 & 65.7 & 66.1 & 66. & & 5.8 & 64.7 & 64.9 & 64.8 \\
\hline National Statis & & & & & & & & & & & & & & & \\
\hline Canada & IUUK & 73.0 & 72 & & 4.0 & 74.6 & 73.7 & 72.6 & 74.3 & 74. & & 3.3 & 70.7 & 72.0 & 72.2 \\
\hline Japan & YXTF & 70.1 & 69 & & 1.3 & 70.8 & 70.9 & 70.0 & 71.3 & 70. & & 0.9 & 69.8 & 70.2 & 70.1 \\
\hline United Kingdom & MGSU & 74.5 & 74 & & 4.5 & 74.6 & 74.8 & 74.8 & 74.8 & 74. & & 4.0 & 73.5 & 72.7 & 72.5 \\
\hline United States & YXTE & 72.2 & 72 & & 1.8 & 71.6 & 71.5 & 71.6 & 71.3 & 70. & & 9.8 & 68.7 & 68.1 & 67.4 \\
\hline & & 2008 & 2008 & 2008 & 2009 & 2009 & 2009 & 2009 & 2009 & 2009 & 2009 & 2009 & 2009 & 2009 & 2009 \\
\hline & & Oct & Nov & Dec & Jan & Feb & Mar & Apr & May & Jun & Jul & Aug & Sep & Oct & Nov \\
\hline Bulgaria & A492 & 5.1 & 5.1 & 5.4 & 5.6 & 6.0 & 6.2 & 6.3 & 6.3 & 6.5 & 6.7 & 6.9 & 7.2 & 7.4 & 7.7 \\
\hline Cyprus & $\mathrm{A} 4 \mathrm{AN}$ & 3.6 & 3.7 & 4.0 & 4.1 & 4.3 & 4.6 & 5.0 & 5.2 & 5.3 & 5.4 & 5.6 & 5.9 & 6.0 & 6.2 \\
\hline Czech Republic & $\mathrm{A} 4 \mathrm{AO}$ & 4.3 & 4.5 & 4.7 & 5.2 & 5.6 & 5.9 & 6.2 & 6.4 & 6.7 & 7.0 & 7.3 & 7.5 & 7.7 & 7.8 \\
\hline Denmark & ZXDJ & 3.5 & 3.7 & 4.0 & 4.4 & 4.7 & 5.3 & 5.8 & 6.0 & 6.2 & 6.1 & 6.1 & 6.5 & 6.9 & 7.1 \\
\hline Estonia & $\mathrm{A} 4 \mathrm{AP}$ & 7.6 & 7.6 & 7.6 & 11.1 & 11.1 & 11.1 & 13.5 & 13.5 & 13.5 & 15.2 & 15.2 & $2 \quad 15.2$ & 15.5 & 15.5 \\
\hline Finland & ZXDU & 6.6 & 6.7 & 6.9 & 7.1 & 7.4 & 7.7 & 8.0 & 8.2 & 8.4 & 8.5 & 8.6 & 8.6 & 8.7 & 8.8 \\
\hline France & ZXDN & 8.1 & 8.3 & 8.5 & 8.7 & 8.9 & 9.0 & 9.2 & 9.3 & 9.4 & 9.5 & 9.6 & 9.7 & 9.9 & 10.0 \\
\hline Germany & $\mathrm{ZXDK}$ & 7.1 & 7.1 & 7.1 & 7.2 & 7.3 & 7.4 & 7.6 & 7.6 & 7.6 & 7.6 & 7.6 & 7.6 & 7.5 & 7.5 \\
\hline Greece & ZXDL & 7.9 & 7.9 & 7.9 & 8.8 & 8.8 & 8.8 & 9.2 & 9.2 & 9.2 & 9.7 & 9.7 & 9.7 & & \\
\hline Hungary & $\mathrm{A} 4 \mathrm{AQ}$ & 7.8 & 8.1 & 8.5 & 8.8 & 9.3 & 9.6 & 9.6 & 9.7 & 9.8 & 10.2 & 10.5 & $5 \quad 10.7$ & 10.8 & 10.8 \\
\hline Ireland & ZXDO & 7.2 & 7.8 & 8.3 & 9.4 & 10.3 & 11.1 & 11.6 & 12.1 & 12.0 & 12.0 & 12.1 & 112.5 & 12.6 & 13.0 \\
\hline Italy & ZXDP & 6.9 & 7.1 & 7.0 & 7.2 & 7.3 & 7.6 & 7.5 & 7.4 & 7.6 & 7.7 & 7.7 & 8.0 & 8.2 & 8.3 \\
\hline Latvia & $\mathrm{A} 4 \mathrm{AR}$ & 9.1 & 10.2 & 11.3 & 12.3 & 13.2 & 14.3 & 15.5 & 16.6 & 17.2 & 18.0 & 18.8 & B 19.9 & 21.1 & 22.0 \\
\hline Lithuania & A4AS & 8.2 & 8.2 & 8.2 & 10.8 & 10.8 & 10.8 & 13.5 & 13.5 & 13.5 & 14.6 & 14.6 & 14.6 & & \\
\hline Luxembourg & ZXDQ & 5.1 & 5.2 & 5.3 & 5.4 & 5.4 & 5.6 & 5.6 & 5.7 & 5.8 & 5.8 & 5.8 & 5.8 & 5.9 & 5.9 \\
\hline Malta & $\mathrm{A} 4 \mathrm{AT}$ & 6.0 & 6.2 & 6.0 & 6.4 & 6.5 & 6.7 & 6.9 & 7.1 & 7.2 & 7.3 & 7.1 & 7.1 & 6.9 & 7.0 \\
\hline Netherlands & ZXDR & 2.7 & 2.7 & 2.7 & 2.8 & 2.9 & 3.1 & 3.2 & 3.3 & 3.4 & 3.5 & 3.7 & 3.8 & 3.9 & 4.0 \\
\hline Poland & $\mathrm{A} 4 \mathrm{AU}$ & 6.8 & 6.9 & 7.0 & 7.4 & 7.7 & 7.9 & 8.0 & 8.0 & 8.1 & 8.3 & 8.4 & 8.5 & 8.6 & 8.7 \\
\hline Portugal & ZXDT & 7.8 & 7.9 & 8.1 & 8.5 & 8.8 & 9.1 & 9.3 & 9.5 & 9.6 & 9.8 & 9.9 & 9 10.1 & 10.2 & 10.3 \\
\hline Romania & A48Z & 5.9 & 5.9 & 5.9 & 6.2 & 6.2 & 6.2 & 6.4 & 6.4 & 6.4 & 7.2 & 7.2 & 7.2 & & \\
\hline Slovak Republic & $\mathrm{A} 4 \mathrm{AV}$ & 8.8 & 9.0 & 9.3 & 9.7 & 10.2 & 10.6 & 10.8 & 11.1 & 11.6 & 12.1 & 12.6 & 13.0 & 13.3 & 13.5 \\
\hline Slovenia & A4AW & 4.2 & 4.2 & 4.2 & 4.6 & 4.9 & 5.3 & 5.6 & 5.9 & 6.2 & 6.4 & 6.4 & 6.5 & 6.8 & 6.8 \\
\hline Spain & ZXDM & 13.1 & 14.0 & 14.8 & 15.8 & 16.7 & 17.4 & 17.7 & 18.0 & 18.1 & 18.4 & 18.7 & $7 \quad 19.0$ & 19.0 & 18.9 \\
\hline Sweden & ZXDV & 6.4 & 7.0 & 6.8 & 6.9 & 7.7 & 7.8 & 8.0 & 8.6 & 8.3 & 8.4 & 8.7 & 8.7 & 8.8 & 8.7 \\
\hline United Kingdom & ZXDW & 6.2 & 6.3 & 6.5 & 6.8 & 7.1 & 7.3 & 7.5 & 7.7 & 7.8 & 7.8 & 7.8 & 7.8 & 7.8 & 7.8 \\
\hline Total EU ${ }^{3}$ & A493 & 7.3 & 7.5 & 7.6 & 8.0 & 8.3 & 8.5 & 8.7 & 8.8 & 8.9 & 9.1 & 9.2 & 9.3 & 9.4 & 9.4 \\
\hline Eurozone $^{3}$ & $\mathrm{ZXDH}$ & 7.8 & 8.0 & 8.2 & 8.5 & 8.8 & 9.1 & 9.2 & 9.3 & 9.4 & 9.5 & 9.6 & 9.8 & 9.8 & 9.9 \\
\hline National Statist & & & & & & & & & & & & & & & \\
\hline Canada & ZXDZ & 6.2 & 6.5 & 6.8 & 7.3 & 8.0 & 8.1 & 8.1 & 8.5 & 8.6 & 8.6 & 8.7 & 8.3 & 8.4 & 8.4 \\
\hline Japan & ZXDY & 3.8 & 4.0 & 4.3 & 4.2 & 4.4 & 4.8 & 5.0 & 5.2 & 5.4 & 5.7 & 5.5 & 5.3 & 5.1 & 5.2 \\
\hline United Kingdom & MGSX & 6.2 & 6.4 & 6.6 & 6.8 & 7.1 & 7.3 & 7.6 & 7.8 & 7.9 & 7.9 & 7.8 & 7.9 & 7.8 & 7.8 \\
\hline United States & $\mathrm{ZXDX}$ & 6.6 & 6.9 & 7.4 & 7.7 & 8.2 & 8.6 & 8.9 & 9.4 & 9.5 & 9.4 & 9.7 & 9.8 & 10.1 & 10.0 \\
\hline
\end{tabular}

1 See chapter text.

3 The "Total EU" series consists of all 27 EU countries. The Eurozone series

2 The UK employment rate as published by the Office for National Statistics is consists of the following EU countries: Austria, Belgium, Cyprus, Finland, seasonally adjusted. All other employment and unemployment rates are not France, Germany, Greece, Ireland, Italy, Luxembourg, Malta, Netherlands, Porseasonally adjusted. 
Labour Market

Full-time equivalents, not seasonally adjusted

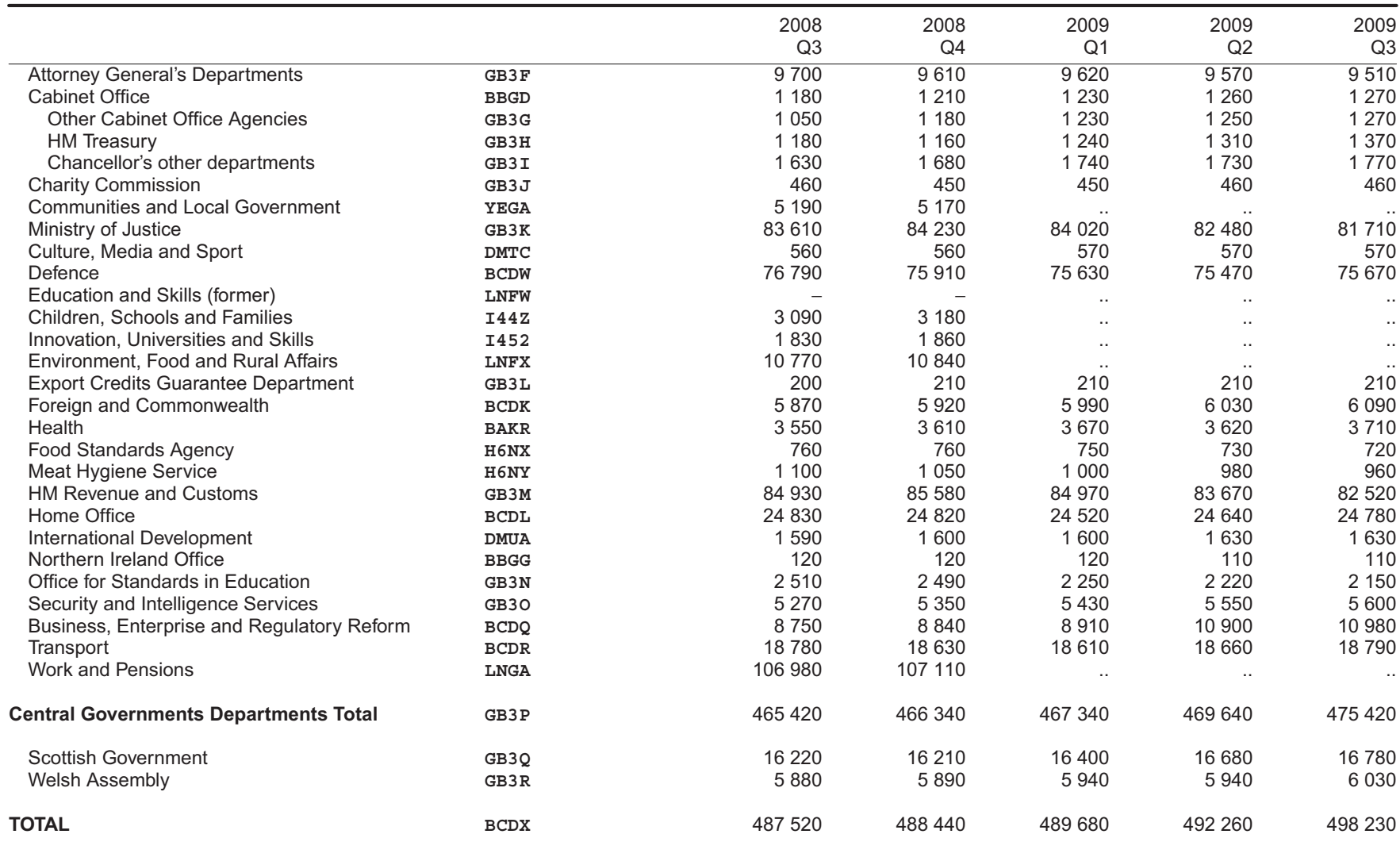

$16-17$

$18-24$

25-34

$35-49$

All

Persons

2003

2004

2005

2006

2007

MGSC

1489

1424

1465

1669
1653

1776

2008
2009

Men

2003

2004
2005

2006

2007

2008

2009

Women

2003

2004

2005

2006

2007

2008

2009

See chapter text.

YBSH
1472
1407
1445
1643
1629
1751
2359


YBSI
891
827
852
955
935
1035
1449


YBSJ
582
580
592
688
695
716
910

YBV
17
1
179
1
197
184
200
YBV
100
100
107
110
101
110
YBVJ

$\begin{array}{rr}\text { YBVH } & \text { YBVN } \\ 172 & 403 \\ 175 & 409 \\ 179 & 438 \\ 183 & 504 \\ 197 & 507 \\ 184 & 558 \\ 200 & 715\end{array}$

YCGM

318

299

285
341

305

YCGS

353

338

419
393

405

327
512

596

MGVL

213

195
190

190
208

223

216

336

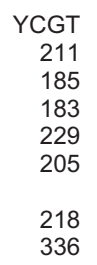

MGVM

145

137
125

134

134
149

147

246

MGVO

18

17
17

27
28

23

23
36

YBVJ YBVP

$\begin{array}{ll}73 & 159 \\ 76 & 169\end{array}$

$\begin{array}{ll}76 & 169 \\ 79 & 168\end{array}$

$76 \quad 201$

$88 \quad 201$

$84 \quad 207$

271

YCGU

MGVN

MGVQ

$\begin{array}{rrr}\text { YCGO } & \text { YCGU } & \text { MGV } \\ 137 & 132 & 67 \\ 114 & 168 & 57 \\ 125 & 154 & 65 \\ 151 & 191 & 73 \\ 143 & 188 & 74 \\ 145 & 187 & 69 \\ 198 & 260 & 90\end{array}$

\section{Q3} 70 770 (n) 10 (5) 


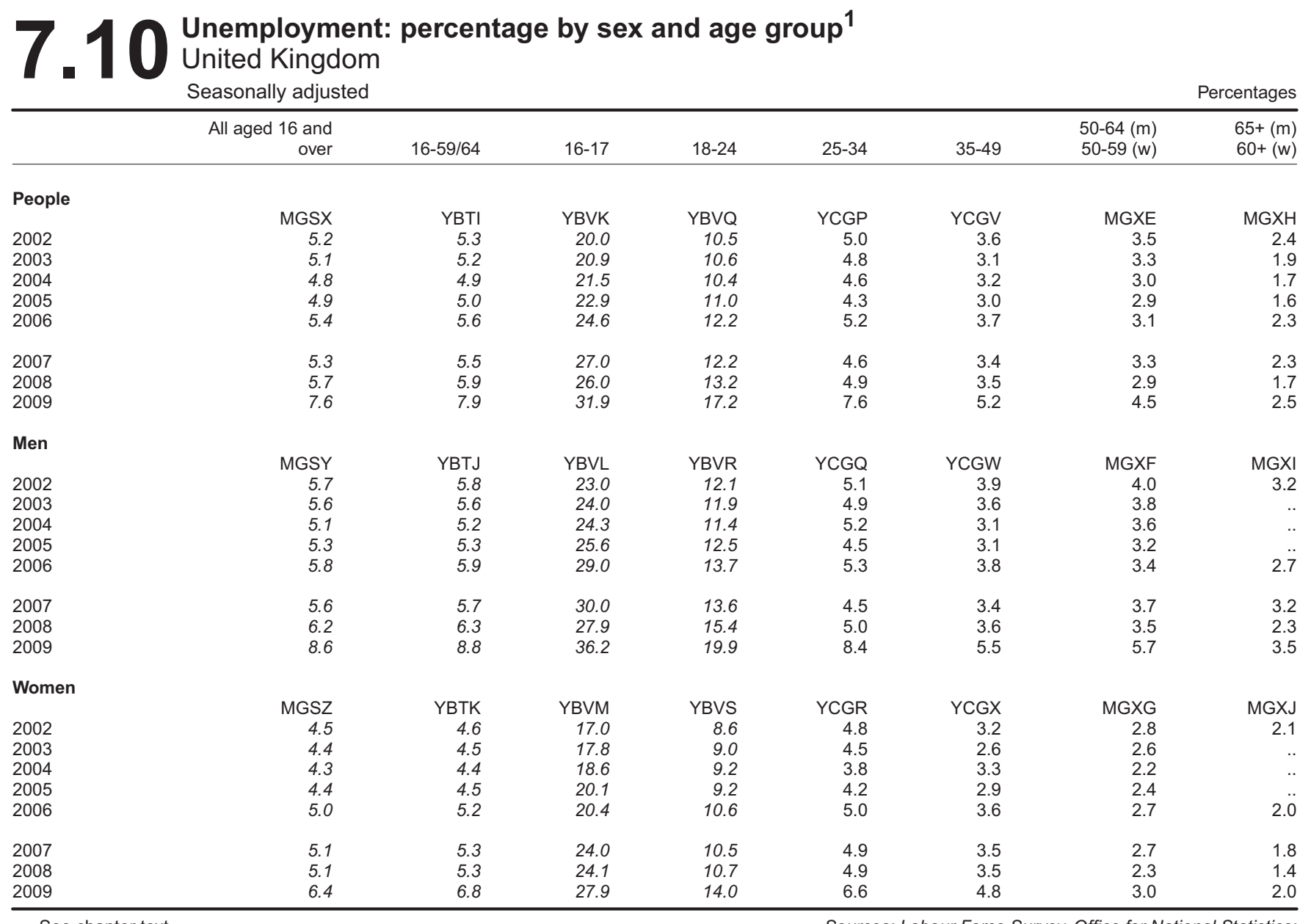

See chapter text.
1 Denominator=economically active for that age group.

Sources: Labour Force Survey, Office for National Statistics;

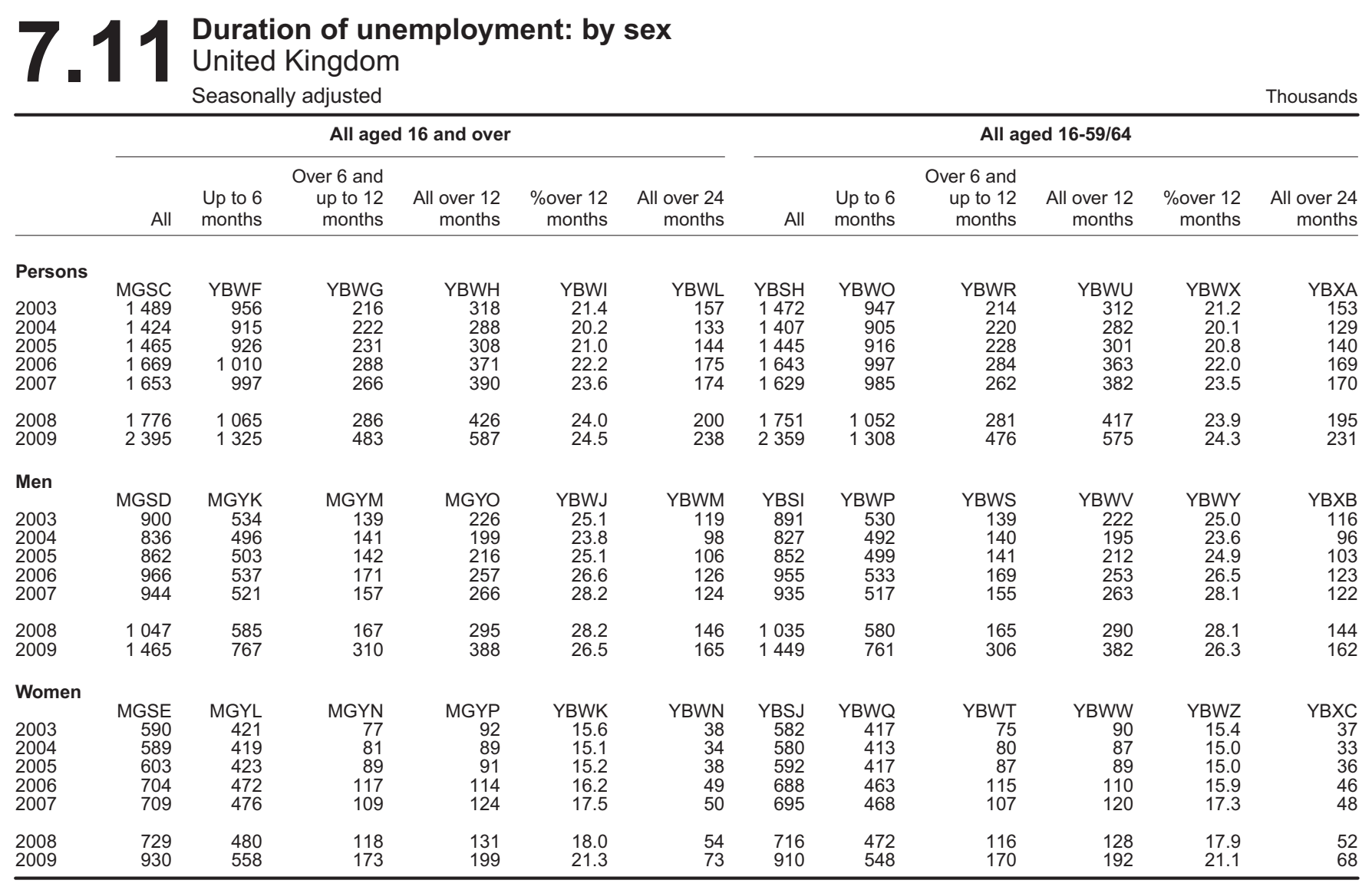




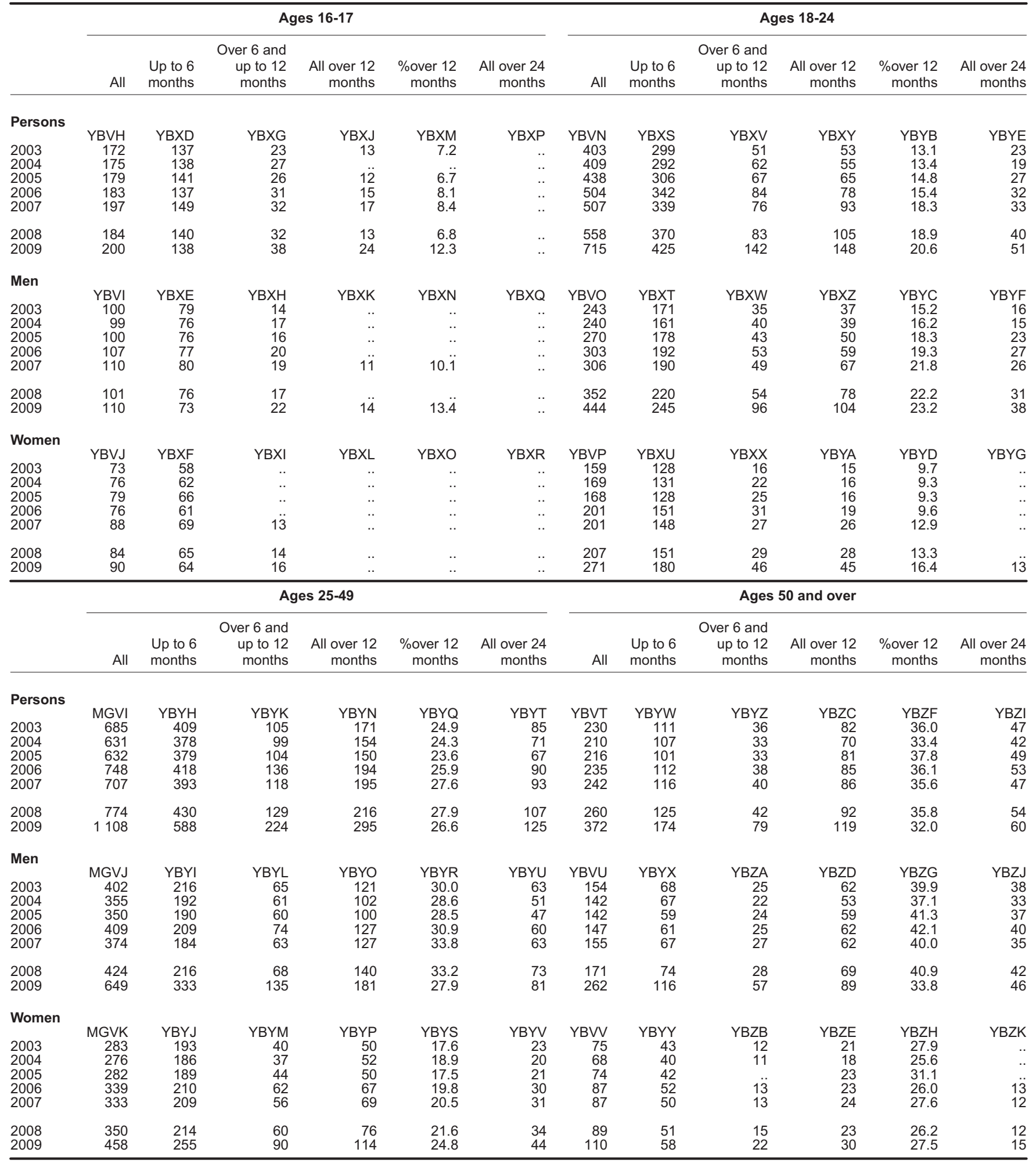

See chapter text. 
Annual averages

Males

All ages

All durations

Up to 6 months

Over 6 and up to 12 months

All over 12 months

All over 24 months

Aged 18 to 24

All durations

Up to 6 months

Over 6 and up to 12 months

All over 12 months

All over 24 months

\section{Aged 25 to 49}

All durations

Up to 6 months

Over 6 and up to 12 months

All over 12 months

All over 24 months

Aged $\mathbf{5 0}$ and over

All durations

Up to 6 months

Over 6 and up to 12 months

All over 12 months

All over 24 months

AGNG
AGXK
ELNP
ELON
IKBS


JLGC
JLGD
JLGE
JLGF

JLGH

AGMA

JLHG

JLHH

JLHI

JLHK

JLHL

JLHM

JLHN

JLHO

JLHQ

Females

\section{All ages}

All durations

Up to 6 months

Over 6 and up to 12 months

All over 12 months

All over 24 months

Aged 18 to 24

All durations

Up to 6 months

Over 6 and up to 12 months

All over 12 months

All over 24 months

\section{Aged 25 to 49}

All durations

Up to 6 months

Over 6 and up to 12 months

All over 12 months

All over 24 months

Aged 50 and ove

All durations

Up to 6 months

Over 6 and up to 12 months

All over 12 months

All over 24 months

JLGI

JLGK

JLGJ

JLGL

JLGN

JLGO

JLGP

JLGO

JLGR

JLGT

JLHR

JLHS

JLHT

JLHU

JLHW

$\begin{array}{rrr}693.0 & 630.7 & 635 \\ 451.2 & 408.8 & 423 \\ 127.1 & 113.7 & 113 . \\ 114.7 & 108.2 & 98 \\ 37.6 & 34.6 & 33.1 \\ & & \\ 171.9 & 161.8 & 174 \\ 143.8 & 134.3 & 143 . \\ 24.5 & 23.3 & 25 \\ 3.6 & 4.2 & 5 \\ 0.4 & 0.5 & 0 . \\ & & \end{array}$

$404.8 \quad 362.3 \quad 357$

$\begin{array}{rrr}248.0 & 221.3 & 225.9 \\ 82.9 & 72.9 & 70.4\end{array}$

82.9

73.8
17.0

72.9

68.1
14.1

70.4
61.2

14.2

$116.2 \quad 106.7$

59.4

19.7
37.2

20.2
103.0

53.9

17.0

32.0

18.3

\section{2}

438.6

136.5

119.1
34.5

34.5

195.6

155.0
33.1

33.1
7.5

0.9

387.

228.9

83.4

75.2

15.2

111.0

54.7
20.0

36.3

18.4 $\begin{array}{lll}628.6 & 662.9 & 1124.2\end{array}$

$475.9 \quad 780.8$

$\begin{array}{rrr}111.2 & 81.4 & 118.2 \\ 33.9 & 21.4 & 18.9\end{array}$

$\begin{array}{lll}176.7 & 191.0 & 321.6\end{array}$

$145.7 \quad 165.0 \quad 263.9$

$\begin{array}{lll}25.3 & 22.0 & 50.9\end{array}$

$\begin{array}{lll}5.8 & 4.0 & 6.8\end{array}$

$352.6 \quad 374.6 \quad 633.5$

$211.6 \quad 250.0 \quad 410.2$

$\begin{array}{lll}69.6 & 67.6 & 138.6\end{array}$

$\begin{array}{llr}71.5 & 57.0 & 84.7\end{array}$

$\begin{array}{lll}14.5 & 11.5 & 12.0\end{array}$

$99.3 \quad 97.3 \quad 169.1$

$\begin{array}{lll}48.0 & 60.9 & 106.7\end{array}$

$\begin{array}{lll}16.2 & 16.0 & 35.7\end{array}$

$\begin{array}{lll}35.0 & 20.4 & 26.7\end{array}$

$18.2 \quad 8.9$

$\begin{array}{rr}230.1 & 214.7 \\ 166.3 & 153.2 \\ 37.2 & 34.9 \\ 26.5 & 26.7 \\ 8.1 & 8.0\end{array}$

$\begin{array}{rr}214.7 & 220.0 \\ 153.2 & 159.2 \\ 34.9 & 35.6 \\ 26.7 & 25.2\end{array}$

159.2
35.6
25.2

246
171
43
30

$\begin{array}{rrr}231.6 & 239.2 & 402.6 \\ 163.8 & 182.1 & 305.0 \\ 37.6 & 34.2 & 67.1 \\ 30.2 & 22.8 & 30.5 \\ 8.4 & 5.8 & 5.3\end{array}$

$\begin{array}{lll}77.3 & 74.0 & 79.1\end{array}$

$\begin{array}{lll}64.9 & 61.5 & 65.3\end{array}$

$\begin{array}{lll}10.6 & 10.4 & 11.4\end{array}$

1.8

0.2

2.1

11.4
2.3

0.4

$\begin{array}{rr}90.2 & 85.6 \\ 72.3 & 71.0 \\ 14.7 & 12.0 \\ 3.3 & 2.5\end{array}$

85.6
71.0
12.0
2.5
0.5

$88.7 \quad 139.2$

$76.6 \quad 116.3$

$\begin{array}{rr}10.2 & 20.2 \\ 1.9 & 2.7\end{array}$

2.7
0.5

$\begin{array}{rr}112.1 & 102 \\ 77.3 & 69.0 \\ 19.9 & 18.2 \\ 14.9 & 14.9 \\ 3.1 & 2.9\end{array}$

$\begin{array}{rr}102.1 & 101 \\ 69.0 & 70.3 \\ 18.2 & 17.7 \\ 14.9 & 13.8 \\ 2.9 & 3.0\end{array}$

101.9
70.3
17.7
13.8
3.0

111.8

73.6

21.3

16.9
3.3

$\begin{array}{rrr}105.4 & 109.9 & 197.3 \\ 69.5 & 78.0 & 144.0 \\ 18.8 & 17.6 & 34.1 \\ 17.1 & 14.2 & 19.3 \\ 3.2 & 2.9 & 3.1\end{array}$

\begin{tabular}{|c|c|c|c|c|c|c|c|}
\hline JLHX & 40.7 & 38.7 & 39.1 & 43.9 & 40.6 & 40.6 & 66.0 \\
\hline JLHY & 24.1 & 22.7 & 23.5 & 25.5 & 23.3 & 27.5 & 44.7 \\
\hline JLHZ & 6.8 & 6.3 & 6.5 & 7.8 & 6.8 & 6.4 & 12.8 \\
\hline JLIA & 9.8 & 9.7 & 9.1 & 10.6 & 10.6 & 6.7 & 8. \\
\hline JLIC & 4.7 & 4.8 & 4.5 & 4.7 & 4.7 & 2.4 & 1.7 \\
\hline
\end{tabular}

1 Count of claimants of unemployment-related benefits. 
At Quarter 2 each year ${ }^{4}$. Seasonally adjusted ${ }^{5}$

Percentages

\begin{tabular}{|c|c|c|c|c|c|c|c|c|c|c|c|c|}
\hline & & 1999 & 2000 & 2001 & 2002 & 2003 & 2004 & 2005 & 2006 & 2007 & 2008 & 2009 \\
\hline North East & YCNC & 9.3 & 8.6 & 7.3 & 6.8 & 6.4 & 5.8 & 6.4 & 6.5 & 6.3 & 7.6 & 9.2 \\
\hline North West & YCND & 6.3 & 5.5 & 5.3 & 5.4 & 4.9 & 4.6 & 4.6 & 5.3 & 5.9 & 6.7 & 8.4 \\
\hline Yorkshire and The Humber & YCNE & 6.3 & 6.1 & 5.3 & 5.3 & 5.1 & 4.7 & 4.8 & 5.8 & 5.6 & 6.1 & 8.6 \\
\hline East Midlands & YCNF & 5.3 & 4.8 & 4.8 & 4.7 & 4.3 & 4.3 & 4.4 & 5.4 & 5.3 & 5.8 & 7.2 \\
\hline West Midlands & YCNG & 6.8 & 6.0 & 5.5 & 5.7 & 5.8 & 5.2 & 4.8 & 5.9 & 6.3 & 6.7 & 9.8 \\
\hline East & $\mathrm{YCNH}$ & 4.2 & 3.7 & 3.7 & 3.8 & 4.0 & 3.7 & 4.1 & 4.8 & 4.7 & 4.8 & 6.3 \\
\hline South East & YCNJ & 3.9 & 3.3 & 3.3 & 3.9 & 3.9 & 3.7 & 3.9 & 4.5 & 4.5 & 4.4 & 5.9 \\
\hline South West & YCNK & 4.5 & 4.1 & 3.6 & 3.8 & 3.4 & 3.3 & 3.6 & 3.8 & 3.9 & 4.1 & 6.3 \\
\hline Wales & YCNM & 7.3 & 6.3 & 5.9 & 5.4 & 4.8 & 4.5 & 4.7 & 5.3 & 5.4 & 6.0 & 8.2 \\
\hline Scotland & YCNN & 7.1 & 6.8 & 6.4 & 6.3 & 5.7 & 5.7 & 5.4 & 5.3 & 4.8 & 4.6 & 7.0 \\
\hline Northern Ireland & ZSFB & 7.1 & 6.3 & 6.0 & 5.8 & 5.5 & 5.0 & 4.6 & 4.4 & 4.0 & 4.5 & 6.5 \\
\hline
\end{tabular}

1 Total unemployed as a percentage of all economically active persons.

2 All aged 16 and over. See chapter text.

Sources: Labour Force Survey, Office for National Statistics,

3 In August 2007, ONS published the mid-year population estimates for 2006.

These estimates have now been incorporated into the LFS estimates from 2001 onwards. Further details can be found at http://www.statistics.gov.uk/cci/article.asp?id=1919

4 The Labour Force Survey has now moved to calendar quarters from May 2006. More information can be found on page 5 of the Concepts and Definitions.pdf by following this link:- www.statistics.gov.uk/downloads/theme labour/Concepts_Definitions_HQS.pdf

5 Previously not seasonally adjusted data was shown.

\section{Claimant count rates: by region ${ }^{1}$ \\ Seasonally adjusted annual averages}

\begin{tabular}{|c|c|c|c|c|c|c|c|c|c|c|c|c|}
\hline & & 1999 & 2000 & 2001 & 2002 & 2003 & 2004 & 2005 & 2006 & 2007 & 2008 & 2009 \\
\hline United Kingdom & BCJE & 4.1 & 3.6 & 3.1 & 3.1 & 3.0 & 2.7 & 2.7 & 3.0 & 2.7 & 2.8 & 4.7 \\
\hline North East & DPDM & 7.0 & 6.3 & 5.6 & 5.1 & 4.5 & 4.0 & 3.9 & 4.1 & 4.0 & 4.5 & 7.0 \\
\hline North West & IBWC & 4.6 & 4.1 & 3.7 & 3.5 & 3.2 & 2.9 & 2.9 & 3.3 & 3.1 & 3.4 & 5.5 \\
\hline Yorkshire and the Humber & DPBI & 5.0 & 4.3 & 3.9 & 3.6 & 3.3 & 2.8 & 2.9 & 3.3 & 3.0 & 3.4 & 5.7 \\
\hline East Midlands & DPBJ & 3.6 & 3.3 & 3.1 & 2.9 & 2.8 & 2.5 & 2.5 & 2.8 & 2.6 & 2.8 & 4.9 \\
\hline West Midlands & DPBN & 4.5 & 4.0 & 3.7 & 3.5 & 3.5 & 3.3 & 3.4 & 3.9 & 3.7 & 3.8 & 6.3 \\
\hline London & DPDQ & 4.4 & 3.7 & 3.3 & 3.6 & 3.6 & 3.5 & 3.4 & 3.5 & 3.0 & 2.8 & 4.3 \\
\hline South East & DPDR & 2.3 & 1.8 & 1.5 & 1.6 & 1.7 & 1.6 & 1.6 & 1.9 & 1.6 & 1.7 & 3.3 \\
\hline South West & DPBM & 3.0 & 2.5 & 2.0 & 1.9 & 1.9 & 1.6 & 1.6 & 1.8 & 1.6 & 1.7 & 3.4 \\
\hline England & VASQ & 3.9 & 3.4 & 3.0 & 2.9 & 2.9 & 2.6 & 2.6 & 2.9 & 2.7 & 2.8 & 4.7 \\
\hline Wales & DPBP & 5.0 & 4.4 & 3.9 & 3.5 & 3.3 & 3.0 & 3.0 & 3.1 & 2.8 & 3.2 & 5.5 \\
\hline Scotland & DPBQ & 5.0 & 4.5 & 3.9 & 3.8 & 3.7 & 3.4 & 3.2 & 3.2 & 2.8 & 2.8 & 4.5 \\
\hline Northern Ireland & DPBR & 6.3 & 5.3 & 4.9 & 4.4 & 4.2 & 3.6 & 3.3 & 3.2 & 2.8 & 3.2 & 5.7 \\
\hline
\end{tabular}

1 The number of unemployment-related benefit claimants as a percentage of

Source: Office for National Statistics: 01633456901

the estimated total workforce (the sum of claimants, employee jobs, self-

employed, participants on work-related government training programmes

and HM Forces) at mid-year. Excluded are claimants under 18, consistent

with current coverage. See chapter text. 


\begin{tabular}{|c|c|c|c|c|c|c|c|c|c|c|c|c|c|c|c|}
\hline & $\begin{array}{r}\text { North } \\
\text { East }\end{array}$ & $\begin{array}{l}\text { North } \\
\text { West }\end{array}$ & $\begin{array}{r}\text { Yorkshire } \\
\text { and the } \\
\text { Humber }\end{array}$ & $\begin{array}{r}\text { East } \\
\text { Midlands }\end{array}$ & $\begin{array}{r}\text { West } \\
\text { Midlands }\end{array}$ & East & London & $\begin{array}{r}\text { South } \\
\text { East }\end{array}$ & $\begin{array}{l}\text { South } \\
\text { West }\end{array}$ & England & Wales & Scotland & $\begin{array}{r}\text { Great } \\
\text { Britain }\end{array}$ & $\begin{array}{l}\text { Northern } \\
\text { Ireland }\end{array}$ & $\begin{array}{r}\text { United } \\
\text { Kingdom }\end{array}$ \\
\hline & DPDG & IBWA & DPAX & DPAY & DPBC & DPDJ & DPDK & DPDL & DPBB & IBWK & DPBE & DPBF & DPAG & DPBG & BCJD \\
\hline 1994 Jan & 145.6 & 325.1 & 233.7 & 174.7 & 262.3 & 210.0 & 451.4 & 296.7 & 203.6 & 2302.3 & 126.7 & 236.0 & 2665.8 & 100.2 & 2766.0 \\
\hline Apr & 141.6 & 314.5 & 227.4 & 170.7 & 252.0 & 200.4 & 440.4 & 280.9 & 194.5 & 2221.7 & 123.3 & 231.7 & 2577.4 & 98.9 & 2676.3 \\
\hline Jul & 139.1 & 304.2 & 222.7 & 166.3 & 242.3 & 191.0 & 428.1 & 268.1 & 188.1 & 2148.8 & 119.0 & 227.4 & 2496.3 & 97.2 & 2593.5 \\
\hline Oct & 136.0 & 291.7 & 215.9 & 160.1 & 230.5 & 180.5 & 415.4 & 251.1 & 178.9 & 2059.0 & 112.9 & 218.1 & 2391.1 & 93.8 & 2484.9 \\
\hline 1995 Jan & 133.0 & 280.1 & 210.6 & 153.2 & 218.5 & 172.3 & 401.4 & 237.9 & 171.4 & 1977.5 & 108.3 & 209.3 & 2296.0 & 91.3 & 2387.3 \\
\hline Apr & 130.0 & 270.8 & 206.8 & 148.1 & 211.0 & 167.1 & 395.0 & 229.7 & 166.0 & 1923.8 & 106.2 & 200.3 & 2231.0 & 88.6 & 2319.6 \\
\hline Jul & 128.2 & 266.1 & 204.6 & 145.3 & 206.9 & 165.0 & 390.2 & 225.1 & 162.5 & 1892.8 & 106.7 & 195.3 & 2195.9 & 87.6 & 2283.5 \\
\hline Oct & 126.4 & 260.9 & 200.7 & 142.3 & 201.3 & 160.7 & 383.2 & 219.1 & 159.4 & 1852.7 & 105.4 & 193.5 & 2152.9 & 85.8 & 2238.7 \\
\hline 1996 Jan & 123.1 & 255.8 & 197.0 & 140.0 & 196.5 & 157.2 & 376.8 & 213.3 & 155.6 & 1814.6 & 104.0 & 193.2 & 2112.5 & 85.9 & 2198.4 \\
\hline Apr & 121.7 & 254.9 & 195.7 & 137.7 & 194.2 & 153.5 & 367.9 & 207.6 & 152.3 & 1785.1 & 104.6 & 194.9 & 2085.0 & 86.1 & 2171.1 \\
\hline Jul & 116.9 & 248.2 & 188.8 & 131.8 & 187.6 & 146.8 & 357.5 & 198.9 & 146.8 & 1722.5 & 101.8 & 191.9 & 2017.0 & 86.4 & 2103.4 \\
\hline Oct & 110.5 & 238.4 & 181.1 & 124.9 & 177.8 & 138.5 & 341.6 & 185.5 & 137.9 & 1635.0 & 98.2 & 186.3 & 1920.7 & 81.7 & 2002.4 \\
\hline 1997 Jan & 101.0 & 218.5 & 166.4 & 111.8 & 160.1 & 123.5 & 312.6 & 163.3 & 126.0 & 1483.2 & 90.3 & 173.8 & 1747.3 & 71.1 & 1818.4 \\
\hline Apr & 95.2 & 201.3 & 154.7 & 102.4 & 147.3 & 110.6 & 284.9 & 144.4 & 112.1 & 1352.9 & 82.5 & 162.2 & 1597.6 & 65.0 & 1662.6 \\
\hline Jul & 92.4 & 188.9 & 148.2 & 95.0 & 138.0 & 102.5 & 264.3 & 131.0 & 100.7 & 1261.0 & 78.1 & 153.6 & 1492.7 & 61.4 & 1554.1 \\
\hline Oct & 90.4 & 177.6 & 142.0 & 87.6 & 131.7 & 94.3 & 246.4 & 120.4 & 93.0 & 1183.4 & 73.6 & 146.5 & 1403.5 & 60.6 & 1464.1 \\
\hline 1998 Jan & 87.6 & 170.6 & 137.2 & 82.8 & 126.1 & 88.5 & 234.3 & 112.3 & 88.7 & 1128.1 & 70.9 & 141.6 & 1340.6 & 59.9 & 1400.5 \\
\hline Apr & 84.1 & 165.4 & 134.1 & 79.9 & 122.3 & 85.2 & 229.4 & 108.0 & 85.1 & 1093.5 & 69.3 & 138.7 & 1301.5 & 57.9 & 1359.4 \\
\hline Jul & 81.8 & 163.7 & 133.3 & 80.0 & 121.4 & 83.7 & 225.2 & 105.5 & 84.1 & 1078.7 & 68.6 & 139.4 & 1286.7 & 57.3 & 1344.0 \\
\hline Oct & 82.1 & 160.9 & 130.9 & 79.9 & 121.4 & 82.0 & 219.3 & 102.5 & 81.8 & 1060.8 & 68.1 & 136.9 & 1265.8 & 56.1 & 1321.9 \\
\hline 1999 Jan & 82.6 & 159.5 & 129.5 & 79.0 & 122.6 & 80.3 & 214.5 & 101.2 & 81.2 & 1050.4 & 67.8 & 135.6 & 1253.8 & 55.9 & 1309.7 \\
\hline Apr & 82.5 & 157.2 & 127.0 & 78.2 & 123.1 & 79.1 & 207.8 & 98.8 & 78.4 & 1032.1 & 67.1 & 133.9 & 1233.1 & 55.0 & 1288.1 \\
\hline Jul & 80.3 & 153.8 & 122.4 & 75.9 & 120.2 & 76.6 & 202.2 & 94.4 & 74.9 & 1000.7 & 63.8 & 130.2 & 1194.7 & 50.0 & 1244.7 \\
\hline Oct & 76.7 & 150.0 & 118.3 & 73.6 & 115.9 & 73.6 & 196.5 & 91.1 & 71.4 & 967.1 & 61.0 & 126.1 & 1154.2 & 46.5 & 1200.7 \\
\hline 2000 Jan & 75.7 & 145.7 & 114.6 & 73.2 & 112.1 & 70.3 & 189.4 & 87.2 & 68.0 & 936.2 & 59.3 & 123.2 & 1118.7 & 44.2 & 1162.9 \\
\hline Apr & 73.6 & 139.9 & 108.9 & 70.0 & 108.1 & 66.9 & 181.6 & 81.3 & 63.8 & 894.1 & 57.8 & 119.0 & 1070.9 & 42.4 & 1113.3 \\
\hline Jul & 72.0 & 135.4 & 104.9 & 68.7 & 107.2 & 62.5 & 172.0 & 77.5 & 61.1 & 861.3 & 57.1 & 115.1 & 1033.5 & 41.2 & 1074.7 \\
\hline Oct & 69.5 & 131.0 & 102.5 & 67.7 & 106.5 & 60.7 & 165.0 & 74.3 & 58.1 & 835.3 & 56.4 & 111.7 & 1003.4 & 41.3 & 1044.7 \\
\hline 2001 Jan & 66.2 & 127.4 & 99.9 & 66.6 & 104.0 & 57.2 & 158.2 & 69.7 & 54.9 & 804.1 & 54.9 & 108.8 & 967.8 & 40.8 & 1008.6 \\
\hline Apr & 63.2 & 124.9 & 97.6 & 65.1 & 100.8 & 54.8 & 151.8 & 66.1 & 53.6 & 777.9 & 52.4 & 105.3 & 935.6 & 39.9 & 975.5 \\
\hline Jul & 61.4 & 121.5 & 95.1 & 63.0 & 97.4 & 53.7 & 151.0 & 65.1 & 52.1 & 760.3 & 49.8 & 102.4 & 912.5 & 39.3 & 951.8 \\
\hline Oct & 61.5 & 121.4 & 93.2 & 61.6 & 95.7 & 54.3 & 156.3 & 65.9 & 51.1 & 761.0 & 49.2 & 104.2 & 914.4 & 38.6 & 953.0 \\
\hline 2002 Jan & 60.9 & 121.3 & 91.4 & 60.6 & 95.4 & 55.4 & 163.1 & 68.6 & 51.1 & 767.8 & 48.1 & 104.3 & 920.2 & 38.0 & 958.2 \\
\hline Apr & 59.2 & 119.4 & 89.4 & 59.4 & 93.6 & 56.4 & 166.2 & 71.0 & 50.9 & 765.5 & 47.5 & 104.4 & 917.4 & 37.5 & 954.9 \\
\hline Jul & 58.5 & 118.1 & 89.1 & 58.6 & 93.4 & 57.5 & 167.3 & 72.3 & 50.1 & 764.9 & 46.8 & 101.9 & 913.6 & 36.4 & 950.0 \\
\hline Oct & 55.9 & 116.1 & 87.6 & 57.9 & 93.7 & 57.2 & 167.6 & 72.3 & 49.3 & 757.6 & 46.7 & 100.1 & 904.4 & 35.1 & 939.5 \\
\hline 2003 Jan & 54.8 & 115.9 & 87.0 & 58.0 & 94.3 & 57.4 & 168.6 & 72.9 & 48.9 & 757.8 & 46.3 & 100.2 & 904.3 & 35.0 & 939.3 \\
\hline Apr & 53.5 & 112.7 & 84.1 & 58.8 & 94.7 & 58.5 & 171.3 & 75.6 & 48.6 & 757.8 & 45.2 & 99.1 & 902.1 & 34.0 & 936.1 \\
\hline Jul & 52.6 & 112.5 & 84.2 & 59.9 & 94.9 & 58.7 & 171.7 & 76.4 & 49.1 & 760.0 & 45.0 & 100.6 & 905.6 & 34.6 & 940.2 \\
\hline Oct & 51.1 & 108.7 & 81.6 & 58.8 & 94.2 & 57.3 & 170.2 & 76.0 & 47.4 & 745.3 & 43.1 & 98.9 & 887.3 & 34.7 & 922.0 \\
\hline 2004 Jan & 49.8 & 104.6 & 78.3 & 56.2 & 93.0 & 56.7 & 167.8 & 75.0 & 45.3 & 726.7 & 42.0 & 97.0 & 865.7 & 33.5 & 899.2 \\
\hline Apr & 47.5 & 101.3 & 75.6 & 53.7 & 89.7 & 56.0 & 165.5 & 72.3 & 42.7 & 704.3 & 41.4 & 94.4 & 840.1 & 31.8 & 871.9 \\
\hline Jul & 45.4 & 96.7 & 71.6 & 51.1 & 86.7 & 54.6 & 162.0 & 68.5 & 40.4 & 677.0 & 39.5 & 90.0 & 806.5 & 29.9 & 836.4 \\
\hline Oct & 45.1 & 96.5 & 71.1 & 50.9 & 86.0 & 55.0 & 158.8 & 69.2 & 40.6 & 673.2 & 39.2 & 89.5 & 801.9 & 29.6 & 831.5 \\
\hline 2005 Jan & 44.0 & 94.4 & 70.1 & 50.8 & 85.7 & 55.1 & 158.8 & 68.4 & 40.9 & 668.2 & 38.9 & 87.4 & 794.6 & 29.1 & 823.7 \\
\hline Apr & 44.9 & 98.0 & 73.2 & 52.1 & 88.0 & 56.4 & 162.1 & 69.7 & 41.5 & 685.9 & 39.6 & 86.1 & 811.7 & 28.6 & 840.3 \\
\hline Jul & 46.1 & 102.2 & 76.1 & 54.5 & 97.1 & 58.9 & 162.4 & 71.6 & 42.5 & 711.4 & 41.7 & 84.9 & 838.1 & 28.6 & 866.7 \\
\hline Oct & 47.4 & 105.9 & 79.9 & 56.5 & 99.2 & 60.2 & 166.0 & 73.7 & 43.0 & 731.8 & 42.8 & 85.8 & 860.0 & 28.2 & 888.2 \\
\hline 2006 Jan & 47.2 & 109.4 & 84.2 & 59.1 & 102.6 & 62.2 & 168.2 & 78.5 & 44.3 & 755.7 & 43.9 & 85.7 & 885.4 & 28.2 & 913.7 \\
\hline Apr & 49.4 & 115.0 & 86.9 & 62.1 & 109.2 & 64.9 & 167.8 & 81.2 & 47.7 & 784.3 & 45.1 & 88.1 & 917.5 & 28.0 & 945.5 \\
\hline Jul & 50.8 & 117.0 & 88.3 & 62.5 & 109.8 & 65.9 & 168.4 & 83.5 & 49.2 & 795.4 & 44.3 & 89.0 & 928.8 & 28.0 & 956.7 \\
\hline Oct & 51.6 & 118.2 & 89.0 & 63.3 & 110.2 & 68.0 & 166.4 & 82.5 & 49.7 & 799.0 & 44.3 & 87.3 & 930.6 & 28.0 & 958.6 \\
\hline 2007 Jan & 51.0 & 115.8 & 86.1 & 62.4 & 109.8 & 66.0 & 159.5 & 78.7 & 47.5 & 776.8 & 42.4 & 82.7 & 901.8 & 26.1 & 927.9 \\
\hline Apr & 49.8 & 111.0 & 83.1 & 59.8 & 104.1 & 62.1 & 151.1 & 74.5 & 43.7 & 739.1 & 41.0 & 78.7 & 858.7 & 24.7 & 883.5 \\
\hline Jul & 49.7 & 108.6 & 80.9 & 58.0 & 100.6 & 60.9 & 143.3 & 70.7 & 42.2 & 714.7 & 40.3 & 75.0 & 830.0 & 23.8 & 853.8 \\
\hline Oct & 48.2 & 108.1 & 78.1 & 56.3 & 100.3 & 58.9 & 137.1 & 67.6 & 40.8 & 695.5 & 40.2 & 72.8 & 808.5 & 23.8 & 832.3 \\
\hline 2008 Jan & 46.9 & 106.0 & 75.4 & 53.3 & 95.1 & 55.5 & 131.8 & 65.8 & 38.0 & 667.4 & 38.6 & 69.6 & 775.7 & 23.7 & 799.3 \\
\hline Apr & 48.1 & 107.4 & 76.9 & 53.7 & 95.5 & 54.7 & 128.3 & 66.0 & 37.8 & 668.5 & 39.7 & 69.9 & 778.1 & 24.2 & 802.3 \\
\hline Jul & 52.2 & 116.0 & 85.0 & 58.8 & 102.2 & 61.3 & 132.9 & 72.9 & 44.9 & 726.4 & 44.0 & 76.3 & 846.6 & 27.1 & 873.7 \\
\hline Oct & 59.8 & 132.4 & 99.1 & 68.7 & 116.2 & 71.9 & 145.9 & 86.8 & 55.4 & 836.4 & 51.2 & 87.9 & 975.4 & 31.5 & 1006.8 \\
\hline 2009 Jan & 72.1 & 161.0 & 123.1 & 89.1 & 144.6 & 92.7 & 170.5 & 117.8 & 73.7 & 1044.7 & 65.7 & 104.3 & 1214.7 & 39.1 & 1253.8 \\
\hline Apr & 82.9 & 188.7 & 147.1 & 107.6 & 172.2 & 116.0 & 205.4 & 147.5 & 93.3 & 1260.7 & 76.9 & 121.8 & 1459.4 & 46.1 & 1505.5 \\
\hline Jul & 86.6 & 198.7 & 154.7 & 111.6 & 179.2 & 120.8 & 219.0 & 155.1 & 96.1 & 1321.8 & 79.4 & 130.7 & 1531.9 & 51.1 & 1583.0 \\
\hline Oct & 88.0 & 203.7 & 160.2 & 115.3 & 185.0 & 123.4 & 229.6 & 160.9 & 96.6 & 1362.7 & 81.3 & 134.7 & 1578.7 & 53.8 & 1632.5 \\
\hline
\end{tabular}
\begin{tabular}{ll}
\hline The figures are based on the number of claimants receiving unemployment & The latest national and regional seasonally adjusted claimant count figures are \\
related benefits and are adjusted for seasonality and discontinuities to be & provisional and subject to revision in the following month.
\end{tabular}

consistent with current coverage. See chapter text. 
Labour Market

Economically active

\begin{tabular}{|c|c|c|c|c|c|c|c|c|}
\hline \multirow{2}{*}{\multicolumn{9}{|c|}{ People }} \\
\hline & MGSF & YBSK & YBZL & YBZO & YBZR & YBZU & YBZX & YCAD \\
\hline 2003 & 29675 & 28713 & 825 & 3817 & 6684 & 10953 & 6434 & 963 \\
\hline 2004 & 29909 & 28893 & 817 & 3936 & 6581 & 11074 & 6485 & 1016 \\
\hline 2005 & 30239 & 29149 & 786 & 3977 & 6584 & 11229 & 6574 & 1090 \\
\hline 2007 & 30875 & 29625 & 731 & 4164 & 6562 & 11437 & 6731 & 1250 \\
\hline 2008 & 31220 & 29871 & 709 & 4221 & 6629 & 11495 & 6816 & 1349 \\
\hline 2009 & 31374 & 29956 & 630 & 4164 & 6734 & 11512 & 6916 & 1419 \\
\hline \multicolumn{9}{|l|}{ Men } \\
\hline & MGSG & YBSL & YBZM & YBZP & YBZS & YBZV & YBZY & YCAE \\
\hline 2003 & 16161 & 15823 & 417 & 2046 & 3664 & 5870 & 3828 & 338 \\
\hline 2004 & 16240 & 15894 & 408 & 2108 & 3593 & 5922 & 3863 & 346 \\
\hline 2005 & 16397 & 16027 & 393 & 2149 & 3592 & 5971 & 3922 & 370 \\
\hline 2006 & 16628 & 16228 & 367 & 2215 & 3605 & 6072 & 3969 & 401 \\
\hline 2007 & 16757 & 16341 & 366 & 2249 & 3611 & 6083 & 4032 & 416 \\
\hline 2008 & 16941 & 16487 & 361 & 2286 & 3635 & 6104 & 4101 & 455 \\
\hline 2009 & 16962 & 16504 & 306 & 2232 & 3718 & 6080 & 4167 & 459 \\
\hline \multicolumn{9}{|c|}{ Women } \\
\hline & MGSH & YBSM & YBZN & YBZQ & YBZT & YBZW & YBZZ & YCAF \\
\hline 2003 & 13515 & 12890 & 408 & 1772 & 3021 & 5084 & 2606 & 625 \\
\hline 2004 & 13669 & 12999 & 409 & 1828 & 2989 & 5151 & 2621 & 670 \\
\hline 2005 & 13842 & 13123 & 393 & 1828 & 2992 & 5258 & 2652 & 720 \\
\hline 2006 & 14070 & 13278 & 375 & 1907 & 2988 & 5319 & 2691 & 792 \\
\hline 2007 & 14118 & 13284 & 365 & 1915 & 2951 & 5354 & 2700 & 834 \\
\hline 2008 & 14278 & 13384 & 348 & 1935 & 2994 & 5391 & 2715 & 894 \\
\hline 2009 & 14412 & 13452 & 324 & 1931 & 3016 & 5432 & 2749 & 960 \\
\hline \multicolumn{9}{|c|}{ Economic activity rates $(\%)^{1}$} \\
\hline \multicolumn{9}{|c|}{ People } \\
\hline & MGWG & MGSO & YCAG & YCAJ & YCAM & YCAP & MGWP & MGWS \\
\hline 2003 & 63.0 & 78.6 & 53.9 & 74.3 & 83.5 & 84.9 & 72.0 & 9.1 \\
\hline 2004 & 63.0 & 78.6 & 52.4 & 74.7 & 83.4 & 84.8 & 72.1 & 9.5 \\
\hline 2005 & 63.2 & 78.6 & 50.0 & 73.7 & 83.8 & 85.0 & 72.6 & 10.1 \\
\hline 2006 & 63.6 & 78.9 & 47.2 & 74.7 & 84.3 & 85.4 & 73.1 & 10.9 \\
\hline 2009 & 63.5 & 79.0 & 40.5 & 71.8 & 84.8 & 85.9 & 74.9 & 12.3 \\
\hline \multicolumn{9}{|l|}{ Men } \\
\hline & MGWH & MGSP & YCAH & YCAK & YCAN & YCAQ & MGWQ & MGWT \\
\hline 2003 & 71.0 & 83.9 & 53.0 & 79.2 & 92.4 & 92.0 & 74.5 & 8.6 \\
\hline 2004 & 70.7 & 83.6 & 51.1 & 79.1 & 91.9 & 91.8 & 74.5 & 8.7 \\
\hline 2005 & 70.6 & 83.5 & 48.8 & 78.6 & 92.3 & 91.4 & 74.9 & 9.2 \\
\hline 2006 & 70.9 & 83.7 & 45.4 & 79.1 & 92.9 & 92.2 & 74.9 & 9.9 \\
\hline 2007 & 70.8 & 83.6 & 44.9 & 78.2 & 93.0 & 91.9 & 75.3 & 10.1 \\
\hline 2008 & 70.9 & 83.8 & 44.3 & 77.9 & 92.8 & 92.2 & 76.0 & 10.9 \\
\hline 2009 & 70.3 & 83.3 & 38.5 & 75.8 & 92.9 & 92.0 & 76.4 & 10.6 \\
\hline \multicolumn{9}{|c|}{ Women } \\
\hline & MGWI & MGSQ & YCAI & YCAL & YCAO & YCAR & MGWR & MGWU \\
\hline 2003 & 55.6 & 73.0 & 54.8 & 69.4 & 74.8 & 77.9 & 68.6 & 9.4 \\
\hline 2004 & 55.8 & 73.2 & 53.9 & 70.1 & 75.1 & 77.9 & 68.8 & 10.0 \\
\hline 2005 & 56.1 & 73.3 & 51.3 & 68.6 & 75.6 & 78.7 & 69.4 & 10.6 \\
\hline 2006 & 56.7 & 73.8 & 49.0 & 70.1 & 75.9 & 78.8 & 70.4 & 11.6 \\
\hline 2007 & 56.5 & 73.8 & 47.3 & 69.3 & 75.6 & 78.8 & 71.4 & 11.9 \\
\hline 2008 & 56.8 & 74.1 & 45.2 & 68.9 & 76.7 & 79.2 & 72.2 & 12.6 \\
\hline 2009 & 56.8 & 74.3 & 42.7 & 67.7 & 76.5 & 80.0 & 72.8 & 13.2 \\
\hline
\end{tabular}

1 Denominator $=$ economically active for that age group. 
All aged 16 and

$16-59 / 64$

$16-17$

$18-24$

25-34

$35-49$

$65+(\mathrm{m})$

over

$50-59(w)$

$60+(w)$

Economically inactive

\section{All Persons}

2003

2004

2005

2006

2007

2008

2009

Men

2003

2004

2005

2006

2007

2008

2009

Women

2003
2004

2005

2006

2007

2008

2009

Economic inactivity rates $(\%)^{1}$

All Persons

2003

2004

2005

2006

2007

2008

2009

Men

2003

2004

2005
2006

2007

2008

2009

Women

2003

2004

2005

2007

2008
2009

MGSI
17411
17538
17632
17570
17793
17839
18093

YBSN
7801
7880
7940
7859
7940
7872
7967

YCAS
705
741
786
831
856

876
923

YBSO

MGSJ

6614
6738

6738
6817

6811

6911

6950

7142

3032

3116

3171

3152

3201

3304

MGSK

10797

10800

10815

10760

10883

10889

10952

\section{YCAT}

369
391

391
412

441

449

453

490

YBSP

4769

4765

4769

4707

4671

4664

YCAU

336
351

351

374

390
406

423

434
YBTL

21.3
21.4

21.4

21.0
21.1

20.9

21.0

YBTM

$\begin{array}{lr}16.1 & \text { LWEY } \\ 16.4 & 47.0\end{array}$

16.4

16.5

16.3

16.4

16.3

29.1

29.6

16.7

YBTE
44.4
44.2
43.9
43.3
43.5

43.3
43.2

LWEX

46.1
47.6

50.0

52.8

52.8
53.9

55.3

59.5

47.0

48.9
51.2

54.6

55.1

55.7

61.5

YBTN

27.0
26.8

26.7

26.1

26.3

\begin{abstract}
25.8
25.7
\end{abstract}
LWEZ

45.2

46.1
48.7

51.0

52.7

54.8
57.3

LWFA

25.7

25.3

26.3

25.4

26.2

26.5

28.2

LWFB

20.8

20.9

21.4

20.9

21.8

22.1

24.2

LWFD

16.5
16.5

16.5

16.2

15.6
15.7

15.3

15.2

LWFG

15.1
15.2

15.0

14.6

14.6
14.8

14.4

14.1

LWFE

7.6
8.1

8.1
7.8

7.8
7.1

7.0

7.2
7.1

LWFC

30.6

29.9

31.4

29.9

30.8

LWFH

8.0
8.2

8.2
8.6

8.6
7.8

8.1

MGWA

2503
2509

2509
2480

2459

2400

2343

2314

MGWD

9610

9659

9692

9854

9967

10126

MGWB

1311
1320

1313

1329

1322

1296

1289

MGWE

3582

3623
3646

3659

3703

3748

3838

MGWC
1192

1189

1166

1130

1079

1047

1025

MGWF

6027

6036

6047

6052

6219

6288

See chapter text.

31.1
32.3

23.4
23.5

LWFF
25.2
24.9
24.5
24.0
24.5

23.4
23.5

8.0

LWFJ

LWFJ
28.0

27.9

27.4

27.0

26.3

LWFM

90.9

89.9

89.1
88.8

88.1

25.6
25.1

87.7

1 Denominator = all persons in the relevant age group.

Sources: Labour Force Survey, Office for National Statistics; 27.8
27.1

LWFK
25.5

25.5

25.1

25.0

24.7

LWFN

91.4
91.3

90.8

90.2
89.9

24.0

89.1

23.6

89.3

Helpline: 01633456901 
Labour Market

\begin{tabular}{|c|c|c|c|c|c|c|c|c|c|}
\hline \multicolumn{7}{|c|}{ Economic inactivity by reason: } & \multicolumn{2}{|c|}{ by: } & \multirow{2}{*}{$\begin{array}{r}\text { All } \\
\text { economically } \\
\text { inactive }\end{array}$} \\
\hline Student & $\begin{array}{l}\text { Looking after } \\
\text { family/home }\end{array}$ & $\begin{array}{r}\text { Temporary } \\
\text { sick }\end{array}$ & $\begin{array}{r}\text { Long-term } \\
\text { sick }\end{array}$ & $\begin{array}{c}\text { Discouraged } \\
\text { workers }^{4}\end{array}$ & Retired & Other & $\begin{array}{r}\text { Does not want } \\
\text { a job }\end{array}$ & Wants a job ${ }^{1}$ & \\
\hline
\end{tabular}

Thousands

All Persons

$\begin{array}{ll} & \text { BEDZ } \\ 2002 & 1543 \\ 2003 & 1655 \\ 2004 & 1717 \\ 2005 & 1836 \\ 2006 & 1837 \\ & \\ 2007 & 1926 \\ 2008 & 1995 \\ 2009 & 2166\end{array}$

BEDZ BEEC

$\begin{array}{ll}655 & 2398 \\ 655 & 2393\end{array}$

$836 \quad 2335$

2325

2315
2267

2243

Men BEEX

2002

2003

2005

2006

2007
2008

2008
2009

Women

2002

2003

2004

2006

2007

2008

763

828

873

913
919

966

1002

1104

BEAQ

180
185

185
189

193

194

197

194
210

BEBL BEBO

779
827

844

844
923

918

959

1061

2073
2034

$\begin{array}{rr}\text { BEBK } & \text { BEBN } \\ 188 & 2203 \\ 187 & 2136 \\ 190 & 2158 \\ 189 & 2126 \\ 191 & 2068 \\ 199 & 2043 \\ 177 & 2019 \\ 173 & 2009\end{array}$

$\begin{array}{rrr}\text { YCFO } & \text { BEEI } & \text { BEEL } \\ 37 & 585 & 810 \\ 35 & 582 & 814 \\ 32 & 595 & 842 \\ 37 & 594 & 825 \\ 37 & 593 & 808 \\ & & \\ 37 & 604 & 815 \\ 42 & 597 & 776 \\ 66 & 578 & 732\end{array}$

BEEL
810
814

814
842

808

2009

BEDI BEDL

$\begin{array}{ll}90 & 1226 \\ 90 & 1170\end{array}$

1170
1180

$\begin{array}{ll}1 & 180 \\ 1 & 171\end{array}$

1134

1131

1118

1114

YCFP

$\begin{array}{rrr}23 & 396 & 337 \\ 20 & 396 & 344\end{array}$

$\begin{array}{ll}396 & 344 \\ 407 & 351\end{array}$

$\begin{array}{ll}414 & 362 \\ 433 & 351\end{array}$

351

443

435
431

352

343
321

BEEJ

977
966

966

978

955
933

912

901

101
91
89

896

Percentages $^{5}$

$\begin{array}{lrr}\text { All Persons } & & \\ & \text { BEDJ } & \text { BED } \\ 2002 & 19.9 & 30.9 \\ 2003 & 21.2 & 30.7 \\ 2004 & 21.8 & 29.8 \\ 2005 & 23.1 & 29.4 \\ 2006 & 23.4 & 29.6 \\ 2007 & 24.3 & 29.3 \\ 2008 & 25.3 & 28.8 \\ 2009 & 27.2 & 28.1 \\ & & \\ \text { Men } & & \text { BEEK } \\ & & 5.9 \\ 2002 & 25.3 & 6.1 \\ 2003 & 27.3 & 6.1 \\ 2004 & 28.0 & 6.1 \\ 2005 & 28.8 & 6.2 \\ 2006 & 29.1 & 6.2 \\ 2007 & 30.1 & 6.1 \\ 2008 & 31.3 & 6.3 \\ 2009 & 33.4 & \end{array}$

$\begin{array}{rrr}\text { BEDM } & \text { BEDP } & \text { BEDS } \\ 30.9 & 2.4 & 28.4 \\ 30.7 & 2.4 & 27.4 \\ 29.8 & 2.4 & 27.4 \\ 29.4 & 2.4 & 26.8 \\ 29.6 & 2.4 & 26.3 \\ & & \\ 29.2 & 2.5 & 25.8 \\ 28.8 & 2.3 & 25.6 \\ 28.1 & 2.2 & 25.2\end{array}$

$\begin{array}{rrr}\text { BEDV } & \text { BEDY } & \text { BEEB } \\ 0.5 & 7.5 & 10.5 \\ 0.4 & 7.5 & 10.4 \\ 0.4 & 7.6 & 10.7 \\ 0.4 & 7.5 & 10.4 \\ 0.5 & 7.6 & 10.3\end{array}$

BEEE
70.9
72.7

72.7

74.1

74.2

73.6

73.6

$\begin{array}{lll}0.4 & 7.6 & 10.3\end{array}$

$\begin{array}{lll}0.5 & 7.6 & 9.9 \\ 0.9 & 7.2 & 9.2\end{array}$

72.8

72.4

BEBM
29.1
27.4
25.9
25.8

BEAR

100

100

100

100

100

26.4

27.2

100

100
100

BEEK BEEN BEEQ

$\begin{array}{rrr}5.9 & 3.0 & 40.7 \\ 6.1 & 3.0 & 38.6\end{array}$

$\begin{array}{lll}6.1 & 3.0 & 38.6 \\ 6.1 & 3.1 & 37.9\end{array}$

6.13 .13 .0

$\begin{array}{lll}6.2 & 3.0 & 36.0\end{array}$

$\begin{array}{lll}6.2 & 3.0 & 35.3\end{array}$

6.3

3.0
2.7

34.9

33.7

BEET BEEW BEEZ

BEAS

BEGT

31.3

29.8

27.8

26.7

72.2

73.3

28.2

BEBP

$\begin{array}{lll}0.8 & 13.0 & 11.4 \\ 0.6 & 13.1 & 11.3\end{array}$

$\begin{array}{lll}0.8 & 13.1 & 11.4\end{array}$

11.1

72.1

27.9

28.6

70.9

29.0

100

100

100

$\begin{array}{lll}0.6 & 13.8 & 11.0 \\ 0.8 & 13.6 & 10.7\end{array}$

$\begin{array}{lll}1.2 & 13.0 & 9.7\end{array}$

70.9

100

100

Women

BEGZ BEHC BEHF BEH

20.5

BEHL BEHO BEBQ

BEHR

BEHU

BEGW

$\begin{array}{rrrrrr}0.3 & 4.0 & 10.0 & 72.3 & 27.7 & 100\end{array}$

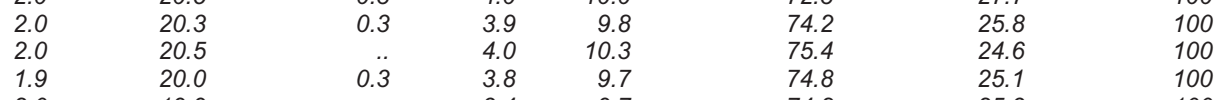

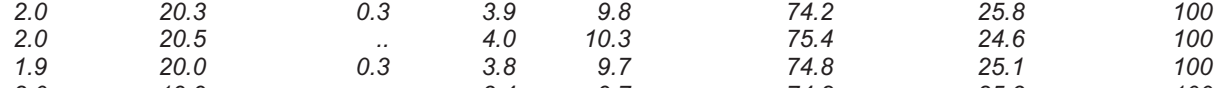

$\begin{array}{lllll}2004 & 17.7 & 45.3 & 2.0 & 20.5 \\ 2005 & 19.3 & 44.9 & 1.9 & 20.0\end{array}$

$\begin{array}{llll}2006 & 19.5 & 45.3 & 2.0\end{array}$

$\begin{array}{lll}. . & 3.4 & 9.7\end{array}$

74.8

25.3

100

\begin{tabular}{lllll}
2007 & 20.3 & 44.8 & 2.2 & 19 \\
2008 & 21.3 & 44.4 & 2.0 & 19.3 \\
2009 & 22.8 & 43.6 & 1.9 & 19.2 \\
\hline
\end{tabular}

1 This series comprises those who have not been looking for work in the last

$\begin{array}{lll}0.4 & 3.4 & 9.8 \\ 0.4 & 3.5 & 9.3\end{array}$

74.6
73.8

25.4

26.2
26.6

100

19.3
19.2

3.2

for National Statistics

Helpline: 01633456901

have been looking for work but who were unable to start within two weeks. 


\section{Labour Market}

\section{Labour disputes: by industry ${ }^{1}$ \\ United Kingdom \\ Standard Industrial Classification 2003}

Thousands and numbers

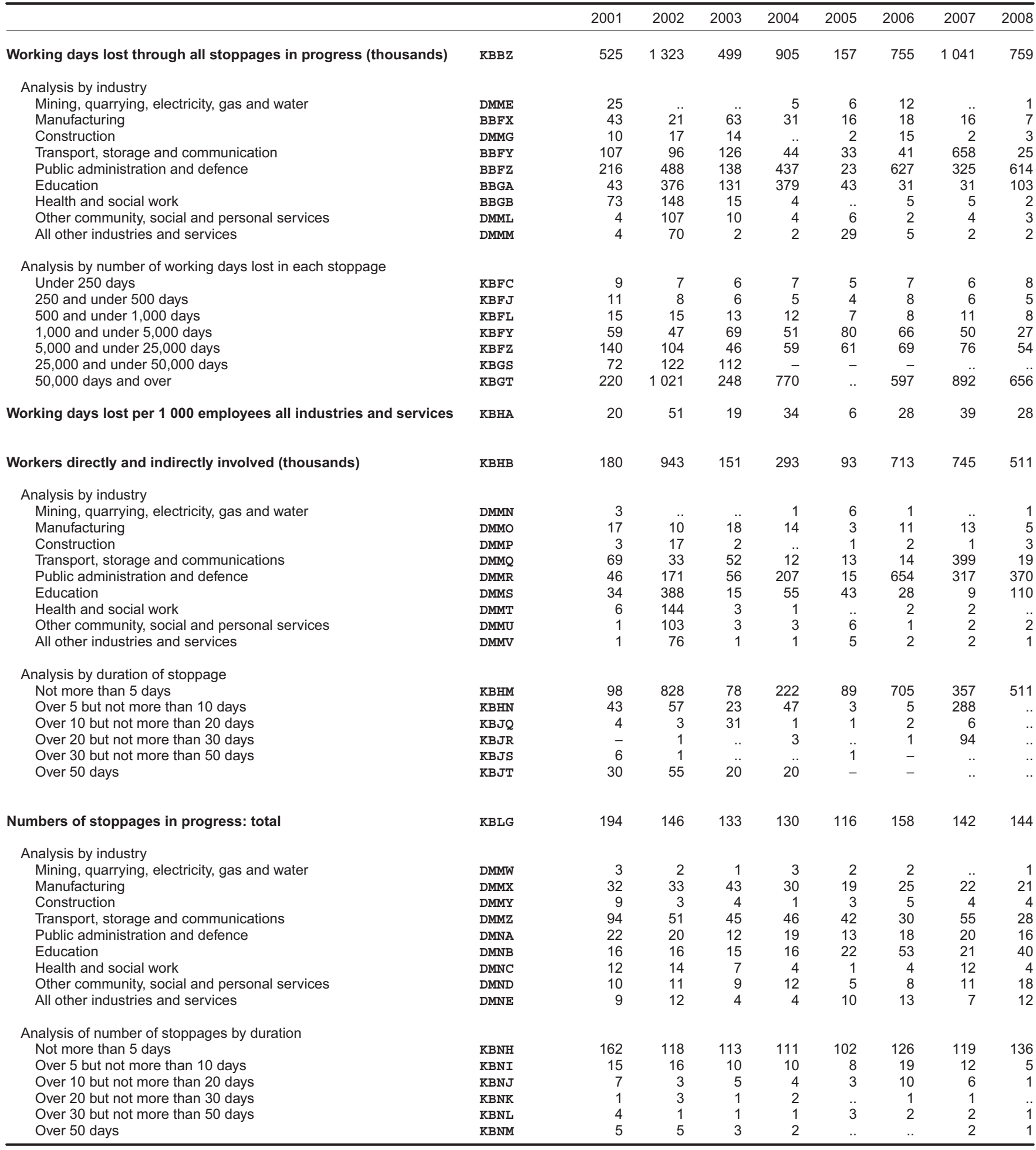

1 See chapter text. 
Labour Market

United Kingdom

April 2008 to 2009. Standard Industrial Classification $2007^{3}$

\begin{tabular}{|c|c|c|c|c|c|c|c|c|}
\hline $\begin{array}{r}\text { Agriculture, } \\
\text { forestry and } \\
\text { fishing }\end{array}$ & $\begin{array}{r}\text { Mining and } \\
\text { Quarrying }\end{array}$ & Manufacturing & $\begin{array}{r}\text { Electricity, } \\
\text { gas, steam and } \\
\text { air } \\
\text { conditioning } \\
\text { supply }\end{array}$ & $\begin{array}{r}\text { Water supply, } \\
\text { sewerage, waste } \\
\text { management and } \\
\text { remediation } \\
\text { activities }\end{array}$ & Construction & $\begin{array}{r}\text { Wholesale and } \\
\text { retail trade; } \\
\text { repair of motor } \\
\text { vehicles and } \\
\text { motorcycles }\end{array}$ & $\begin{array}{r}\text { Transport and } \\
\text { storage }\end{array}$ & $\begin{array}{r}\text { Accommodation } \\
\text { and food } \\
\text { service } \\
\text { activities }\end{array}$ \\
\hline
\end{tabular}

Median gross weekly earnings

All employees

$\begin{array}{lrrr} & & \\ 2008 & \text { JR89 } & \text { JR8A } & \text { JR8 } \\ 2009 & 356.8 & 649.0 & 486.3\end{array}$

$2009 \quad 439.0 \quad 409.7$

Men

2008

$\begin{array}{lll}\text { JR8S } & \text { JR8T } & \text { JR8U } \\ 375.4 & 674.8 & 513.6\end{array}$

2009

402.5

726.5

510.4

Women

2008

$\begin{array}{ll}\text { JR9D } & \text { JR9E } \\ 301.1 & 499.6\end{array}$

JR9F

2009

345.0

568.0

370.5

Median hourly earnings (excluding overtime)
All employees

JR9W JR9X

$\begin{array}{lrr}2008 & 7.69 & 15.27\end{array}$

$\begin{array}{lll}2009 & 8.24 & 16.67\end{array}$

Men

2008

2009

JRB9 JRC2

$\begin{array}{ll}7.69 & 15.27\end{array}$

$8.24 \quad 16.67$

Women

2008

2009

JRE4 JRE5

JRE4
7.21

8.25

15.07

16.17

Median total paid hours worked

All employees

2008
2009

JS5P

2009

40.0

JS5Q

40.0

39.9
37.5

Men

2008

JS6A

2009

$\begin{array}{ll}40.0 & 39.9\end{array}$

Women

2008

JS6T JS6U

JS6T JS6U

$\begin{array}{ll}49.1 & 37.0\end{array}$

2009

\section{JR9Y}

11.44

11.76

JRC3

11.44

11.76

JRE6

9.20

9.50

10.53

10.68

JR9Z

16.12

14.97

JRA2

10.94

11.80

JRA3

11.79

12.22

JRC5

10.94

11.80

JRC6

JRC4
16.12

16.12
14.97

11.79

12.22

JRE8

11.03

12.23

JRE9

10.49

10.50

JR8E

592.9

380.0

482.2

JR8G

470.6

375.9

JR8H

296.8

R8X

533.3
550.0

JR8Y

421.6

428.0

JR8Z

480.1

488.3

JR9J

317.7

325.8

JR9K

421.6

436.5

567.

JR92

316.2

317.3

JR9L

272.9

278.3

JS5R
39.1
38.9


JS6C
39.8
39.0


JS6V
37.8
37.5

JS5S

37.0

JS5T

40.0

JS5U

40.0

40.0

JS5V

39.1

JS5W

40.0

40.0

40.0

40.0

JS6D

37.2

JS6E

JS6F

$\begin{array}{ll}40.5 & 40.0 \\ 40.7 & 40.0\end{array}$

JS6G

40.0

40.0

JS6H

40.5

JS6

40.0

JS6W

37.0

JS6X

37.2

JS6Y

37.5

37.5
37.5

JS6Z

37.6

37.6

JS72

38.9

38.9
37.5

JS73

39.8
39.5 
continued
Median ${ }^{1}$ weekly and hourly earnings of full-time employees ${ }^{2}$ by industry division ${ }^{3}$
Anited Kingdom 2008 to 2009 . Standard Industrial Classification $2007^{3}$

\begin{tabular}{|c|c|c|c|c|c|c|c|c|c|}
\hline $\begin{array}{r}\text { Information } \\
\text { and } \\
\text { communication }\end{array}$ & $\begin{array}{r}\text { Financial and } \\
\text { insurance } \\
\text { activities }\end{array}$ & $\begin{array}{r}\text { Real estate } \\
\text { activities }\end{array}$ & $\begin{array}{r}\text { Professional } \\
\text { and } \\
\text { scientific } \\
\text { and technical } \\
\text { activities }\end{array}$ & $\begin{array}{r}\text { Administrati- } \\
\text { ve and } \\
\text { support } \\
\text { service } \\
\text { activities }\end{array}$ & $\begin{array}{r}\text { Public } \\
\text { administrati- } \\
\text { on and } \\
\text { defence }\end{array}$ & Education & $\begin{array}{r}\text { Human Health } \\
\text { and social } \\
\text { work } \\
\text { activities }\end{array}$ & $\begin{array}{l}\text { Arts,enterta- } \\
\text { inment and } \\
\text { recreation }\end{array}$ & $\begin{array}{r}\text { Other service } \\
\text { activities }\end{array}$ \\
\hline
\end{tabular}

Median gross weekly earnings

All employees

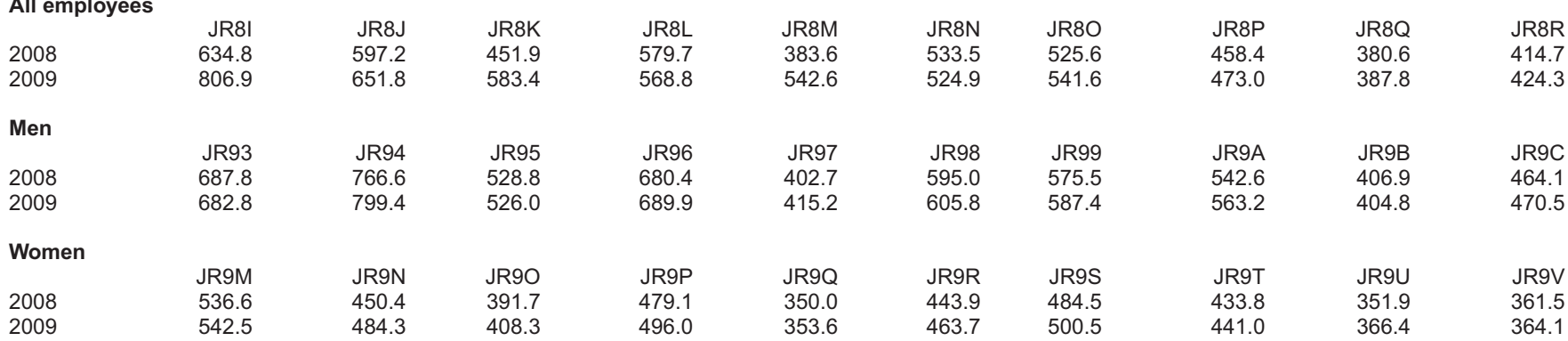

Median hourly earnings (excluding overtime)

All employees

$\begin{array}{lll} & \text { JRA7 } & \text { JRA } \\ 2008 & 16.39 & 16.43 \\ 2009 & 16.73 & 17.38 \\ & & \\ \text { Men } & & \\ & & \\ 2008 & \text { JRD2 } & \text { JRD } \\ 2009 & 16.39 & 16.43 \\ & 16.73 & 17.3 \\ \text { Women } & & \\ & & \\ 2008 & \text { JRF5 } & \text { JRF } \\ 2009 & 14.23 & 12.45 \\ & 14.64 & 13.5\end{array}$

Median total paid hours worked

All employees

$\begin{array}{lrrrrr}\text { All employees } & & & & \\ & \text { JS5Y } & \text { JS5Z } & \text { JS62 } & \text { JS63 } & \text { JS64 } \\ 2008 & 37.5 & 35.0 & 37.4 & 37.5 & 40.0 \\ 2009 & 37.5 & 35.0 & 37.0 & 37.5 & 39.9 \\ \text { Men } & & & & & \\ 2008 & \text { JS6J } & \text { JS6K } & \text { JS6L } & \text { JS6M } & \text { JS6N } \\ 2009 & 37.5 & 35.0 & 37.5 & 37.5 & 40.0 \\ \text { Women } & 37.5 & 35.0 & 37.4 & 37.5 & 40.0 \\ 2008 & & & & & \\ 2009 & \text { JS74 } & \text { JS75 } & \text { JS76 } & \text { JS77 } & \text { JS78 } \\ & 37.5 & 35.0 & 37.0 & 37.0 & 37.5 \\ & 37.5 & 35.0 & 37.0 & 37.0 & 37.5 \\ & & & & & \end{array}$

1 See chapter text. Median values are less affected by extremes of earnings at either ends of the scale with half the employees earning above the stated amount and half below. Previous editions of Annual Abstract published means.

2 Data relate to full-time employeeson adult rates whose pay for the surveyperiod was not affected by absence.

3 Classification is based on Standard Industrial Classification 2007.
Sources: Annual Survey of Hours and Earnings; Office for National Statistics: 01633456120 


\begin{tabular}{|c|c|c|c|c|c|c|c|c|}
\hline & \multicolumn{4}{|c|}{ Manufacturing industries } & \multicolumn{4}{|c|}{ All industries and services } \\
\hline & \multirow[b]{2}{*}{$\begin{array}{r}\text { Gross } \\
\text { weekly } \\
\text { earnings }\end{array}$} & \multirow[b]{2}{*}{$\begin{array}{r}\text { Total } \\
\text { paid } \\
\text { hours }\end{array}$} & \multicolumn{2}{|c|}{ Hourly earnings $(£)$} & \multirow[b]{2}{*}{$\begin{array}{r}\text { Gross } \\
\text { weekly } \\
\text { earnings }(£)\end{array}$} & \multirow[b]{2}{*}{$\begin{array}{r}\text { Total } \\
\text { paid } \\
\text { hours }\end{array}$} & \multicolumn{2}{|c|}{ Hourly earnings(£) } \\
\hline & & & $\begin{array}{r}\text { including } \\
\text { overtime } \\
\text { pay }\end{array}$ & $\begin{array}{r}\text { excluding } \\
\text { overtime } \\
\text { pay }\end{array}$ & & & $\begin{array}{l}\text { including } \\
\text { overtime }\end{array}$ & $\begin{array}{c}\text { excluding } \\
\text { overtime }\end{array}$ \\
\hline \multicolumn{9}{|c|}{ SIC $2007^{3}$} \\
\hline \multicolumn{9}{|l|}{ All } \\
\hline & JR7J & JR7K & JR7L & JR7M & JR7V & JR7W & JR7X & JR7Y \\
\hline 2008 & 486.3 & 39.1 & 11.72 & 11.44 & 479.1 & 37.5 & 11.98 & 11.88 \\
\hline 2009 & 484.9 & 38.9 & 11.94 & 11.76 & 488.7 & 37.5 & 12.43 & 12.34 \\
\hline \multicolumn{9}{|l|}{ Men } \\
\hline & JR7N & JR7O & JR7P & JR7Q & JR7Z & JR82 & JR83 & JR84 \\
\hline 2008 & 513.6 & 39.8 & 12.21 & 11.95 & 522.0 & 39.0 & 12.63 & 12.50 \\
\hline 2009 & 510.4 & 39.0 & 12.47 & 12.27 & 531.1 & 38.7 & 13.09 & 12.97 \\
\hline \multicolumn{9}{|c|}{ Women } \\
\hline 2009 & 370.5 & 37.5 & 9.54 & 9.50 & 426.4 & 37.0 & 11.42 & 11.40 \\
\hline
\end{tabular}

1 Median values are less affected by extremes of earnings at either ends of the scale with half the employees earning above the stated amount and half below. Previous editions of Annual Abstract published means.

2 Data relate to full-time employees on adult rates whose pay for the survey

pay-period was not affected by absence.

3 Classification is based on Standard Industrial Clasification 2007.

\subsection{2 \\ Average weekly earnings: main industrial sectors \\ Great Britain}

Standard Industrial Classification 2007

\begin{tabular}{|c|c|c|c|c|c|c|c|c|c|c|}
\hline & \multicolumn{2}{|c|}{ Whole economy } & \multicolumn{2}{|c|}{ Manufacturing } & \multicolumn{2}{|c|}{ Construction } & \multicolumn{2}{|c|}{ Services } & \multicolumn{2}{|c|}{$\begin{array}{c}\text { Distribution Hotels and } \\
\text { Restuarants }\end{array}$} \\
\hline & Actual & $\begin{array}{r}\text { Seasonally } \\
\text { adjusted }\end{array}$ & Actual & $\begin{array}{r}\text { Seasonally } \\
\text { adjusted }\end{array}$ & Actual & $\begin{array}{r}\text { Seasonally } \\
\text { adjusted }\end{array}$ & Actual & $\begin{array}{r}\text { Seasonally } \\
\text { adjusted }\end{array}$ & Actual & $\begin{array}{r}\text { Seasonally } \\
\text { adjusted }\end{array}$ \\
\hline & KA46 & KAB9 & KA49 & KAE3 & $\mathrm{KA} 4 \mathrm{C}$ & KAE6 & KA4F & KAD2 & KA4I & KAE9 \\
\hline 2000 & 321 & 320 & 371 & 371 & 386 & 386 & 306 & 306 & 224 & 224 \\
\hline 2001 & 338 & 337 & 385 & 385 & 418 & 417 & 323 & 322 & 233 & 233 \\
\hline 2003 & 359 & 359 & 414 & 413 & 445 & 445 & 345 & 344 & 247 & 247 \\
\hline 2004 & 375 & 374 & 434 & 433 & 458 & 458 & 360 & 360 & 255 & 255 \\
\hline 2005 & 392 & 391 & 450 & 449 & 472 & 471 & 378 & 377 & 264 & 264 \\
\hline 2006 & 410 & 410 & 466 & 465 & 501 & 501 & 396 & 396 & 274 & 273 \\
\hline 2007 & 431 & 430 & 484 & 483 & 535 & 535 & 416 & 416 & 290 & 290 \\
\hline 2008 & 446 & 445 & 500 & 499 & 546 & 545 & 433 & 431 & 297 & 297 \\
\hline 2009 & 445 & 445 & 506 & 505 & 553 & 553 & 431 & 430 & 301 & 301 \\
\hline
\end{tabular}

\begin{tabular}{|c|c|c|c|c|c|c|c|c|}
\hline & \multicolumn{2}{|c|}{ Finance and Business Industries } & \multicolumn{2}{|c|}{ Private Sector } & \multicolumn{2}{|c|}{ Public Sector } & \multicolumn{2}{|c|}{$\begin{array}{c}\text { Private Sector Excl Financial } \\
\text { Services }\end{array}$} \\
\hline & Actual & $\begin{array}{r}\text { Seasonally } \\
\text { adjusted }\end{array}$ & Actual & $\begin{array}{r}\text { Seasonally } \\
\text { adjusted }\end{array}$ & Actual & $\begin{array}{l}\text { Seasonally } \\
\text { adjusted }\end{array}$ & Actual & $\begin{array}{l}\text { Seasonally } \\
\text { adjusted }\end{array}$ \\
\hline & KA4L & KAD5 & KA4O & KAC4 & KA4R & KAC7 & KA4U & KAD8 \\
\hline 2000 & 410 & 412 & 322 & 322 & 314 & 313 & 314 & 313 \\
\hline 2001 & 443 & 443 & 339 & 339 & 331 & 329 & 331 & 329 \\
\hline 2002 & 451 & 452 & 349 & 349 & 344 & 342 & 344 & 342 \\
\hline 2003 & 463 & 464 & 359 & 359 & 360 & 358 & 360 & 358 \\
\hline 2004 & 486 & 487 & 374 & 374 & 376 & 374 & 375 & 374 \\
\hline 2005 & 511 & 511 & 391 & 390 & 396 & 394 & 395 & 394 \\
\hline 2006 & 546 & 546 & 410 & 410 & 410 & 408 & 410 & 408 \\
\hline 2007 & 574 & 572 & 432 & 432 & 424 & 421 & 424 & 421 \\
\hline 2008 & 603 & 600 & 448 & 447 & 439 & 437 & 438 & 436 \\
\hline 2009 & 583 & 584 & 443 & 443 & 453 & 451 & 450 & 448 \\
\hline
\end{tabular}




\begin{tabular}{|c|c|c|c|c|c|c|c|c|c|}
\hline $\begin{array}{r}\text { Agriculture, } \\
\text { forestry } \\
\text { and } \\
\text { fishing }\end{array}$ & $\begin{array}{r}\text { Mining } \\
\text { and } \\
\text { quarrying }\end{array}$ & $\begin{array}{r}\text { Food } \\
\text { products, } \\
\text { beverages } \\
\text { and } \\
\text { tobacco }\end{array}$ & $\begin{array}{r}\text { Textiles, } \\
\text { leather and } \\
\text { clothing }\end{array}$ & $\begin{array}{r}\text { Chemicals } \\
\text { and man- } \\
\text { made fibres }\end{array}$ & $\begin{array}{r}\text { Basic metals } \\
\text { and metal } \\
\text { products }\end{array}$ & $\begin{array}{r}\text { Engineering } \\
\text { and allied } \\
\text { industries }\end{array}$ & $\begin{array}{r}\text { Other } \\
\text { manufacturing }\end{array}$ & $\begin{array}{r}\text { Electricity, } \\
\text { gas and } \\
\text { water supply }\end{array}$ & Construction \\
\hline
\end{tabular}

Excluding bonuses

SIC 1992

\begin{tabular}{|c|c|c|c|c|c|c|c|c|c|c|}
\hline & JT7X & JT7Y & JT7Z & JT82 & JT83 & JT84 & JT85 & JT86 & JT87 & JT88 \\
\hline 2008 & 303 & 883 & 436 & 346 & 562 & 476 & 526 & 448 & 584 & 522 \\
\hline 2009 & 314 & 915 & 446 & 360 & 602 & 475 & 527 & 458 & 608 & 53 \\
\hline 2007 Aug & 283 & 856 & 421 & 357 & 561 & 452 & 510 & 425 & 554 & 51 \\
\hline Sep & 278 & 835 & 418 & 355 & 564 & 453 & 510 & 430 & 557 & 52 \\
\hline Oct & 280 & 854 & 426 & 353 & 566 & 459 & 514 & 435 & 571 & 517 \\
\hline Nov & 281 & 859 & 422 & 340 & 575 & 458 & 515 & 435 & 567 & 521 \\
\hline Dec & 277 & 871 & 431 & 340 & 570 & 451 & 514 & 438 & 576 & 51 \\
\hline 2008 Jan & 289 & 875 & 418 & 344 & 566 & 479 & 518 & 443 & 569 & 509 \\
\hline Feb & 299 & 878 & 431 & 355 & 565 & 469 & 518 & 447 & 569 & 51 \\
\hline Mar & 302 & 859 & 436 & 343 & 550 & 465 & 530 & 450 & 584 & 5 \\
\hline Apr & 303 & 862 & 435 & 344 & 565 & 475 & 531 & 445 & 569 & 523 \\
\hline May & 304 & 888 & 434 & 345 & 557 & 476 & 527 & 448 & 571 & 52 \\
\hline Jun & 303 & 900 & 438 & 342 & 566 & 478 & 526 & 449 & 569 & 52 \\
\hline Jul & 298 & 881 & 435 & 345 & 563 & 481 & 529 & 448 & 583 & 525 \\
\hline Aug & 302 & 882 & 434 & 343 & 550 & 477 & 525 & 444 & 618 & 311 \\
\hline Nov & 306 & 883 & 443 & 354 & 562 & 486 & 529 & 454 & 598 & 530 \\
\hline Dec & 310 & 910 & 452 & 346 & 570 & 474 & 529 & 449 & 604 & 52 \\
\hline 2009 Jan & 311 & 885 & 447 & 354 & 578 & 479 & 523 & 448 & 592 & 53 \\
\hline Feb & 301 & 904 & 442 & 359 & 574 & 471 & 521 & 447 & 595 & 53 \\
\hline Mar & 314 & 904 & 449 & 357 & 585 & 471 & 525 & 451 & 607 & 53 \\
\hline Apr & 308 & 924 & 449 & 361 & 587 & 478 & 527 & 453 & 596 & 53 \\
\hline May & 322 & 916 & 447 & 359 & 612 & 475 & 522 & 460 & 605 & 53 \\
\hline Jun & 319 & 912 & 445 & 354 & 622 & 474 & 522 & 460 & 607 & 52 \\
\hline Jul & 303 & 922 & 444 & 356 & 616 & 463 & 520 & 456 & 612 & 532 \\
\hline Aug & 314 & 906 & 435 & 359 & 609 & 467 & 527 & 459 & 607 & 1 \\
\hline Sep & 319 & 925 & 443 & 355 & 593 & 474 & 527 & 461 & 607 & 534 \\
\hline Oct & 313 & 927 & 438 & 366 & 610 & 475 & 536 & 464 & 616 & 540 \\
\hline Nov & 323 & 928 & 437 & 371 & 613 & 484 & 537 & 465 & 629 & 540 \\
\hline Dec & 325 & 930 & 476 & 373 & 623 & 486 & 541 & 466 & 626 & \\
\hline 2010 Jan & 321 & 905 & 475 & 379 & 620 & 478 & 536 & 476 & 622 & \\
\hline Feb & 309 & 937 & 457 & 370 & 620 & 505 & 536 & 471 & 620 & \\
\hline
\end{tabular}

Percentage change on the year

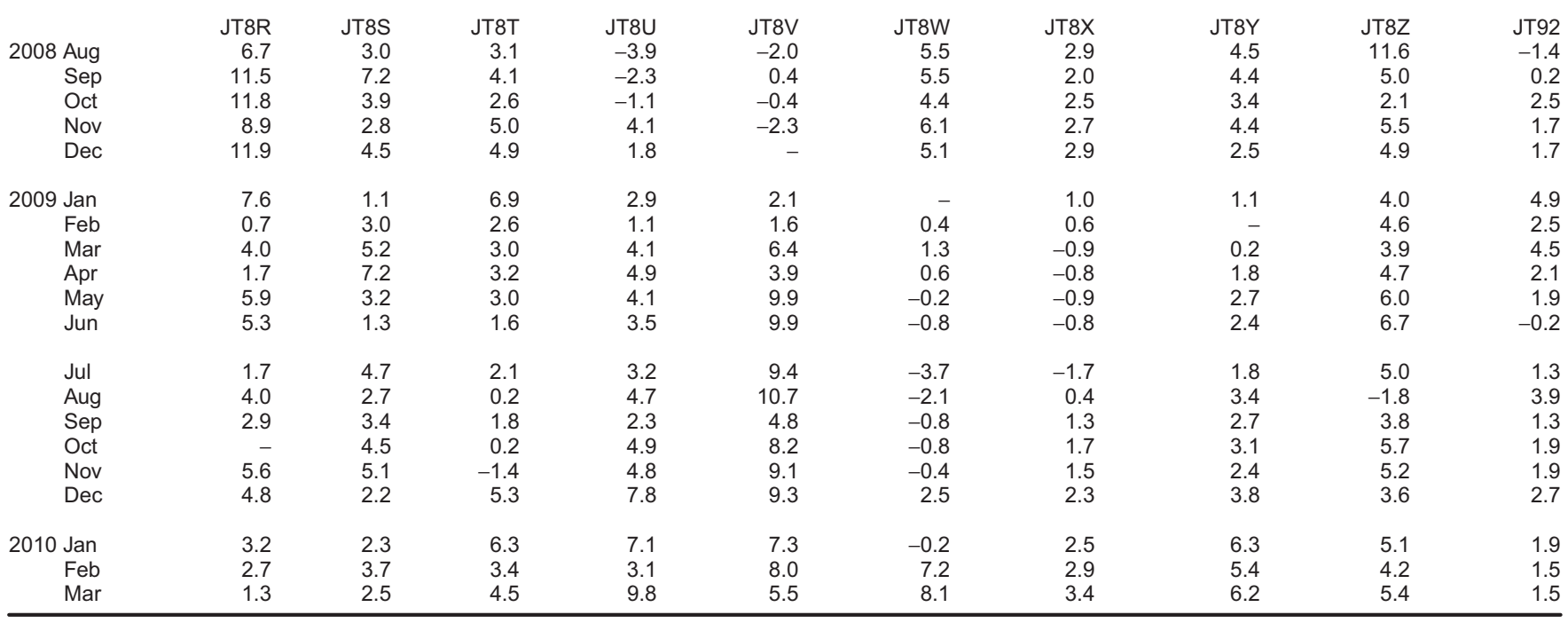


Labour Market

\begin{tabular}{|c|c|c|c|c|c|c|c|c|c|}
\hline $\begin{array}{r}\text { Wholesale } \\
\text { trade }\end{array}$ & $\begin{array}{l}\text { Retail trade } \\
\text { and repairs }\end{array}$ & $\begin{array}{l}\text { Hotels and } \\
\text { restaurants }\end{array}$ & $\begin{array}{r}\text { Transport, } \\
\text { storage and } \\
\text { communication }\end{array}$ & $\begin{array}{r}\text { Financial } \\
\text { interm- } \\
\text { ediation }\end{array}$ & $\begin{array}{r}\text { Real estate } \\
\text { renting and } \\
\text { business } \\
\text { activities }\end{array}$ & $\begin{array}{l}\text { Public } \\
\text { admini- } \\
\text { stration }\end{array}$ & Education & $\begin{array}{l}\text { Health and } \\
\text { social work }\end{array}$ & $\begin{array}{r}\text { Other } \\
\text { services }\end{array}$ \\
\hline
\end{tabular}

Excluding bonuses

SIC 1992

2008
2009

$\begin{array}{rrr}\text { JT89 JT8A } & \text { JT8B } \\ 472 & \text { JT }\end{array}$

480

256

209

209

JT8C JT8D

$\begin{array}{ll}497 & 656 \\ 502 & 686\end{array}$

JT8E

JT8F

686

482

509

JT8G
377

390

$\begin{array}{rr}\text { JT8H } & \text { JT8 } \\ 383 & 370\end{array}$

2007 Aug

$\begin{array}{ll}455 & 248 \\ 452 & 246 \\ 461 & 242 \\ 455 & 239 \\ 458 & 238\end{array}$

494

633
638

635

453

Oct

208
207
209

Nov

213

483
481
487

487

638

453
456

490

496

640

459

461

485

485

496

368
371
368

2008 Jan

Feb
Mar
Apr

470
466

247

206

May

472

243

206

247
256

Jun

471
471

252

214

207

Jul

Aug

Oct

Nov

470

468
475

473

474

492
493

493
500

500
502
501

636

646
654

654
654

654
653
662

461

465

464

471
469

496

497

490
496

496
494

501

$248 \quad 208$

$\begin{array}{ll}251 & 212 \\ 252 & 210\end{array}$

$249-209$

Dec

245

$$
204
$$

490
492

492
494

499

497
499

671
657

657
653

663

659

475

494

$479 \quad 495$

$\begin{array}{ll}484 & 496 \\ 480 & 495\end{array}$

$480 \quad 495$

$\begin{array}{ll}481 & 507 \\ 479 & 503\end{array}$

2009 Jan

Feb
Mar
Apr

480

479

254

205

254

256

256
258

May

476
478

257

209

209

210

211
209

500

497

498
501

501
504

505

664

675

675

689

683
682

$480 \quad 502$

$482 \quad 524$

482

488

$\begin{array}{ll}487 & 510 \\ 487 & 504\end{array}$

$499 \quad 685$

$257 \quad 211$

Jul

Sep

Oct

$479 \quad 257$

478
478

484

259

210
208

Dec

484
488

252

503
505

505

502

685
689

690

693

696

485

483
480

480
478

478
478

506

509

503

512

$479 \quad 511$

$503 \quad 699$

483

519

484
487

502
511

718
723

487

518

$\begin{array}{rr}\text { JT98 } & \text { JT99 } \\ 6.8 & 1.2 \\ 6.0 & 1.6 \\ 5.3 & 2.1 \\ 4.8 & 3.5 \\ 3.9 & 1.4\end{array}$

JT9A

2.7
3.0

3.0
3.5

6.2

4.5

$392 \quad 366$

Percentage change on the year

2008 Au

$\begin{array}{rr}2.9 & 1.2 \\ 5.1 & 2.4 \\ 2.6 & 2.9 \\ 4.2 & 2.5 \\ 4.4 & 4.2\end{array}$

-4.2
-1.4

3.7
2.1

$\begin{array}{ll}0.6 & 4.2\end{array}$

$\begin{array}{ll}4.1 & 1.2 \\ 3.7 & 5.4\end{array}$

$\begin{array}{ll}1.6 & 4.4 \\ 0.8 & 4.5\end{array}$

$-0.4$

$-0.2$

0.6

3.2

5.4

4.6
3.0

$\begin{array}{ll}3.7 & 5.4 \\ 3.9 & 3.3\end{array}$

$\begin{array}{ll}3.9 & 2.8\end{array}$

$3.8 \quad 3.2$

$1.8 \quad 2.1$

1.8
2.2

2.2

1.2

2.1
4.9

5.7

4.5

Oct

Nov

2.3

2.1

3.2

2.9

2.0

1.4

1.0
1.2

8.3
4.3

$1.3 \quad 2.2$

$\begin{array}{rr}1.3 & 2.2 \\ -0.2 & 2.6\end{array}$

$\begin{array}{rr}- & 1.6 \\ -0.4 & 2.6\end{array}$

$\begin{array}{ll}-0.4 & 2.6 \\ -0.6 & 1.0\end{array}$

2010 Jan

$\begin{array}{rrr}0.8 & 2.8 & 2.4 \\ - & 2.0 & 2.9\end{array}$

1.5

3.1

2.9

$\begin{array}{ll}0.6 & 5.3 \\ 1.0 & 6.4\end{array}$

2.6

7.1

$\begin{array}{rr}0.6 & 3.4 \\ 0.4 & -0.8\end{array}$

$\begin{array}{rr}0.4 & -0.8 \\ 1.0 & 2.4\end{array}$

\begin{tabular}{rrr}
5.5 & 3.2 & -0.5 \\
5.2 & 2.1 & -1.1 \\
4.1 & 2.9 & -2.9 \\
4.3 & 3.1 & 0.5 \\
3.8 & 4.2 & -2.7 \\
4.8 & 5.0 & -0.8 \\
& & \\
4.8 & 2.3 & -2.6 \\
4.2 & -0.8 & -2.9 \\
3.9 & 2.1 & 0.3 \\
3.9 & 1.8 & -0.5 \\
-0.8 & 1.0 & - \\
- & 1.3 & 0.5 \\
& & \\
0.8 & 0.5 & 5.5 \\
0.5 & 2.9 & 1.9 \\
1.6 & 1.0 & 1.4 \\
\hline
\end{tabular}




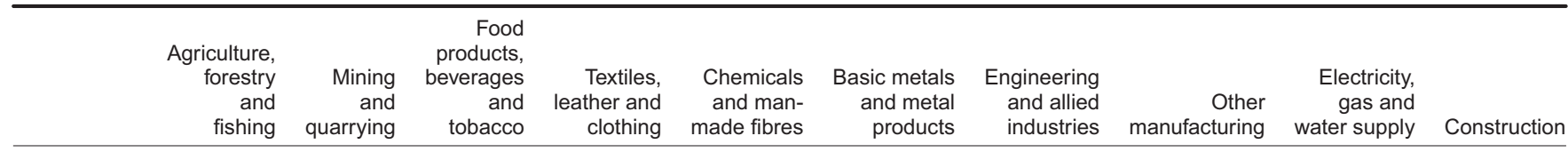

Including bonuses

SIC 1992

\begin{tabular}{|c|c|c|c|c|c|c|c|c|c|c|}
\hline & KA5Z & KA64 & KA67 & $\mathrm{KA} 6 \mathrm{~A}$ & KA6D & KA6G & KA6J & KA6M & KA6P & KA6S \\
\hline 2008 & 310 & 996 & 460 & 368 & 600 & 499 & 547 & 469 & 622 & 546 \\
\hline 2009 & 321 & 1013 & 466 & 384 & 641 & 492 & 548 & 475 & 652 & 553 \\
\hline Sep & 282 & 923 & 437 & 391 & 591 & 468 & 519 & 440 & 582 & 555 \\
\hline Oct & 282 & 906 & 437 & 368 & 582 & 480 & 525 & 450 & 595 & 538 \\
\hline Nov & 286 & 946 & 429 & 357 & 588 & 479 & 531 & 452 & 593 & 555 \\
\hline Dec & 287 & 991 & 461 & 362 & 613 & 504 & 549 & 473 & 591 & 584 \\
\hline 2008 Jan & 297 & 957 & 428 & 369 & 586 & 499 & 534 & 458 & 587 & 531 \\
\hline Feb & 300 & 987 & 469 & 370 & 603 & 491 & 556 & 466 & 594 & 539 \\
\hline Mar & 311 & 1384 & 488 & 374 & 696 & 492 & 591 & 492 & 697 & 561 \\
\hline Apr & 308 & 965 & 457 & 355 & 667 & 506 & 546 & 466 & 628 & 541 \\
\hline May & 308 & 951 & 482 & 356 & 569 & 497 & 544 & 470 & 598 & 539 \\
\hline Jun & 305 & 999 & 456 & 402 & 593 & 495 & 543 & 469 & 627 & 548 \\
\hline Jul & 300 & 957 & 462 & 357 & 580 & 520 & 543 & 471 & 629 & 541 \\
\hline Aug & 309 & 920 & 442 & 350 & 558 & 492 & 535 & 457 & 635 & 527 \\
\hline Sep & 318 & 972 & 458 & 364 & 576 & 489 & 532 & 461 & 621 & 553 \\
\hline Nov & 308 & 943 & 450 & 369 & 588 & 497 & 540 & 468 & 623 & 553 \\
\hline Dec & 346 & 984 & 479 & 378 & 607 & 501 & 564 & 483 & 619 & 567 \\
\hline 2009 Jan & 318 & 1004 & 457 & 369 & 596 & 497 & 539 & 460 & 617 & 547 \\
\hline Feb & 311 & 995 & 458 & 375 & 607 & 493 & 556 & 460 & 625 & 546 \\
\hline Mar & 321 & 1298 & 520 & 387 & 753 & 505 & 587 & 487 & 731 & 576 \\
\hline Apr & 311 & 1031 & 465 & 378 & 711 & 499 & 554 & 468 & 651 & 553 \\
\hline May & 323 & 972 & 456 & 374 & 622 & 489 & 537 & 473 & 633 & 543 \\
\hline Jun & 321 & 972 & 460 & 452 & 644 & 489 & 535 & 476 & 673 & 543 \\
\hline Jul & 313 & 969 & 459 & 367 & 631 & 484 & 533 & 474 & 667 & 548 \\
\hline Aug & 316 & 970 & 442 & 365 & 615 & 475 & 536 & 472 & 631 & 543 \\
\hline Sep & 326 & 967 & 464 & 365 & 601 & 480 & 537 & 474 & 634 & 550 \\
\hline Oct & 318 & 981 & 447 & 375 & 622 & 486 & 545 & 479 & 643 & 555 \\
\hline Nov & 326 & 977 & 448 & 389 & 625 & 507 & 547 & 479 & 663 & 560 \\
\hline Dec & 352 & 1024 & 511 & 411 & 669 & 506 & 568 & 494 & 651 & 574 \\
\hline 2010 Jan & 325 & 993 & 484 & 402 & 644 & 499 & 548 & 492 & 649 & 560 \\
\hline Feb & 330 & 1092 & 472 & 390 & 682 & 533 & 566 & 495 & 648 & 554 \\
\hline
\end{tabular}

Percentage change on the year

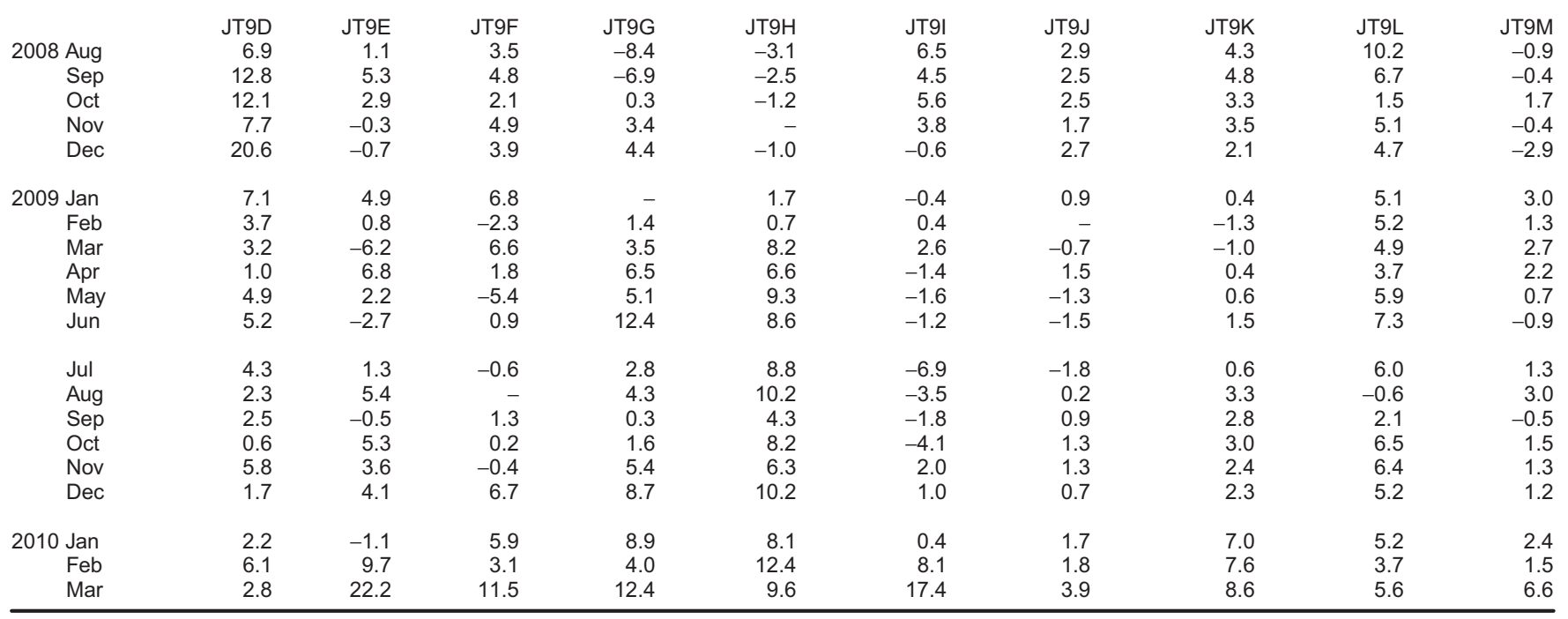


Labour Market

\begin{tabular}{|c|c|c|c|c|c|c|c|c|c|}
\hline $\begin{array}{r}\text { Wholesale } \\
\text { trade }\end{array}$ & $\begin{array}{l}\text { Retail trade } \\
\text { and repairs }\end{array}$ & $\begin{array}{l}\text { Hotels and } \\
\text { restaurants }\end{array}$ & $\begin{array}{r}\text { Transport, } \\
\text { storage and } \\
\text { communication }\end{array}$ & $\begin{array}{r}\text { Financial } \\
\text { interm- } \\
\text { ediation }\end{array}$ & $\begin{array}{r}\text { Real estate } \\
\text { renting and } \\
\text { business } \\
\text { activities }\end{array}$ & $\begin{array}{l}\text { Public } \\
\text { admini- } \\
\text { stration }\end{array}$ & Education & $\begin{array}{l}\text { Health and } \\
\text { social work }\end{array}$ & $\begin{array}{r}\text { Other } \\
\text { services }\end{array}$ \\
\hline
\end{tabular}

Including bonuses

SIC 1992

\begin{tabular}{|c|c|c|c|c|c|c|c|c|c|c|}
\hline & KA6V & KA6Y & KA73 & KA76 & KA79 & KA7C & KA7F & KA7I & KA7L & KA7O \\
\hline 2008 & 517 & 264 & 214 & 525 & 970 & 507 & 500 & 378 & 384 & 387 \\
\hline 2009 & 520 & 271 & 214 & 526 & 886 & 512 & 511 & 391 & 393 & 383 \\
\hline 2007 Aug & 486 & 259 & 212 & 511 & 714 & 482 & 502 & 369 & 365 & 386 \\
\hline Sep & 487 & 259 & 210 & 494 & 739 & 479 & 488 & 372 & 366 & 382 \\
\hline Oct & 495 & 259 & 214 & 492 & 681 & 480 & 486 & 369 & 369 & 372 \\
\hline Nov & 502 & 252 & 219 & 502 & 707 & 484 & 495 & 373 & 383 & 375 \\
\hline Dec & 512 & 252 & 224 & 531 & 780 & 508 & 506 & 378 & 377 & 378 \\
\hline 2008 Jan & 516 & 258 & 209 & 505 & 1402 & 490 & 497 & 367 & 377 & 387 \\
\hline Feb & 530 & 259 & 216 & 519 & 1984 & 498 & 498 & 368 & 377 & 383 \\
\hline Mar & 558 & 277 & 225 & 534 & 1390 & 525 & 492 & 369 & 378 & 406 \\
\hline Apr & 513 & 276 & 210 & 521 & 740 & 506 & 499 & 373 & 381 & 385 \\
\hline May & 504 & 270 & 218 & 564 & 785 & 498 & 495 & 374 & 379 & 384 \\
\hline Jun & 518 & 267 & 212 & 571 & 801 & 507 & 496 & 373 & 381 & 385 \\
\hline Jul & 510 & 264 & 212 & 510 & 744 & 521 & 505 & 377 & 386 & 396 \\
\hline Aug & 501 & 262 & 215 & 513 & 724 & 514 & 507 & 379 & 393 & 394 \\
\hline Sep & 505 & 261 & 212 & 505 & 752 & 502 & 498 & 383 & 385 & 377 \\
\hline Nov & 521 & 257 & 209 & 511 & 706 & 503 & 511 & 396 & 390 & 378 \\
\hline Dec & 526 & 261 & 216 & 531 & 870 & 516 & 510 & 394 & 391 & 389 \\
\hline 2009 Jan & 520 & 266 & 207 & 512 & 1028 & 508 & 503 & 387 & 390 & 386 \\
\hline Feb & 541 & 273 & 216 & 513 & 1235 & 517 & 527 & 386 & 385 & 379 \\
\hline Mar & 551 & 282 & 214 & 537 & 1182 & 539 & 507 & 384 & 389 & 394 \\
\hline Apr & 511 & 274 & 213 & 525 & 807 & 517 & 511 & 389 & 394 & 387 \\
\hline May & 508 & 273 & 218 & 564 & 761 & 513 & 511 & 388 & 396 & 376 \\
\hline Jun & 514 & 276 & 212 & 549 & 851 & 513 & 505 & 390 & 399 & 380 \\
\hline Jul & 512 & 273 & 214 & 514 & 757 & 513 & 509 & 394 & 395 & 388 \\
\hline Aug & 507 & 271 & 212 & 521 & 751 & 505 & 512 & 396 & 389 & 378 \\
\hline Sep & 505 & 269 & 210 & 516 & 788 & 502 & 504 & 398 & 394 & 376 \\
\hline Oct & 512 & 271 & 212 & 516 & 756 & 499 & 509 & 397 & 395 & 376 \\
\hline Nov & 522 & 265 & 214 & 517 & 783 & 499 & 515 & 393 & 394 & 383 \\
\hline Dec & 536 & 264 & 220 & 525 & 927 & 515 & 521 & 394 & 397 & 388 \\
\hline 2010 Jan & 527 & 275 & 214 & 515 & 895 & 509 & 520 & 390 & 392 & 405 \\
\hline Feb & 539 & 283 & 223 & 522 & 1709 & 519 & 521 & 389 & 396 & 389 \\
\hline
\end{tabular}

Percentage change on the year

\begin{tabular}{|c|c|c|c|c|c|c|c|c|c|c|}
\hline & JT9N & JT9O & JT9P & JT9Q & JT9R & JT9S & JT9T & JT9U & JT9V & JT9W \\
\hline 2008 Aug & 3.1 & 1.2 & 1.4 & 0.4 & 1.4 & 6.6 & 1.0 & 2.7 & 7.7 & 2.1 \\
\hline Sep & 3.7 & 0.8 & 1.0 & 2.2 & 1.8 & 4.8 & 2.0 & 3.0 & 5.2 & -1.3 \\
\hline Oct & 1.4 & 0.8 & -1.4 & 4.1 & 8.1 & 5.6 & 2.1 & 3.5 & 5.1 & 1.6 \\
\hline Nov & 3.8 & 2.0 & -4.6 & 1.8 & -0.1 & 3.9 & 3.2 & 6.2 & 1.8 & 0.8 \\
\hline 2009 Jan & 0.8 & 3.1 & -1.0 & 1.4 & -26.7 & 3.7 & 1.2 & 5.4 & 3.4 & -0.3 \\
\hline Feb & 2.1 & 5.4 & - & -1.2 & -37.8 & 3.8 & 5.8 & 4.9 & 2.1 & -1.0 \\
\hline Mar & -1.3 & 1.8 & -4.9 & 0.6 & -15.0 & 2.7 & 3.0 & 4.1 & 2.9 & -3.0 \\
\hline May & 0.8 & 1.1 & - & - & -3.1 & 3.0 & 3.2 & 3.7 & 4.5 & -2.1 \\
\hline Jun & -0.8 & 3.4 & - & -3.9 & 6.2 & 1.2 & 1.8 & 4.6 & 4.7 & -1.3 \\
\hline Jul & 0.4 & 3.4 & 0.9 & 0.8 & 1.7 & -1.5 & 0.8 & 4.5 & 2.3 & -2.0 \\
\hline Aug & 1.2 & 3.4 & -1.4 & 1.6 & 3.7 & -1.8 & 1.0 & 4.5 & -1.0 & -4.1 \\
\hline Sep & - & 3.1 & -0.9 & 2.2 & 4.8 & - & 1.2 & 3.9 & 2.3 & -0.3 \\
\hline Oct & 2.0 & 3.8 & 0.5 & 0.8 & 2.7 & -1.6 & 2.6 & 3.9 & 1.8 & -0.5 \\
\hline Nov & 0.2 & 3.1 & 2.4 & 1.2 & 10.9 & -0.8 & 0.8 & -0.8 & 1.0 & 1.3 \\
\hline Dec & 1.9 & 1.1 & 1.9 & -1.1 & 6.6 & -0.2 & 2.2 & - & 1.5 & -0.3 \\
\hline Feb & -0.4 & 3.7 & 3.2 & 1.8 & 38.4 & 0.4 & -1.1 & 0.8 & 2.9 & 2.6 \\
\hline Mar & 12.7 & 10.3 & 3.7 & 5.0 & 13.1 & 3.3 & 2.8 & 1.6 & 1.0 & 7.1 \\
\hline
\end{tabular}




\begin{tabular}{|c|c|c|c|c|c|c|c|c|c|c|}
\hline & \multicolumn{5}{|c|}{ Gross weekly earnings } & \multicolumn{5}{|c|}{ Gross hourly earnings } \\
\hline & $\begin{array}{c}\text { Lowest } \\
\text { decile }\end{array}$ & $\begin{array}{l}\text { Lower } \\
\text { quartile }\end{array}$ & Median & $\begin{array}{l}\text { Upper } \\
\text { quartile }\end{array}$ & $\begin{array}{r}\text { Highest } \\
\text { decile }\end{array}$ & $\begin{array}{c}\text { Lowest } \\
\text { decile }\end{array}$ & $\begin{array}{l}\text { Lower } \\
\text { quartile }\end{array}$ & Median & $\begin{array}{l}\text { Upper } \\
\text { quartile }\end{array}$ & $\begin{array}{r}\text { Highest } \\
\text { decile }\end{array}$ \\
\hline \multicolumn{11}{|c|}{ All employees } \\
\hline & C5U9 & C5UC & C5UF & C5UI & C5UL & C5UO & C5UR & C5UU & $\mathrm{C} 5 \mathrm{~V} 2$ & C5UX \\
\hline 2006 & 243.8 & 315.2 & 443.6 & 630.5 & 881.6 & 6.24 & 7.93 & 11.12 & 16.39 & 23.49 \\
\hline 2007 & 252.9 & 325.8 & 457.6 & 650.5 & 907.1 & 6.47 & 8.22 & 11.47 & 16.87 & 24.17 \\
\hline 2008 & 262.2 & 338.8 & 479.1 & 677.9 & 950.7 & 6.67 & 8.51 & 11.98 & 17.59 & 25.12 \\
\hline 2009 & 270.6 & 347.5 & 488.7 & 692.8 & 971.0 & 6.95 & 8.83 & 12.43 & 18.19 & 25.90 \\
\hline \multicolumn{11}{|c|}{ Male employees } \\
\hline & C5UA & C5UD & C5UG & C5UJ & C5UM & C5UP & C5US & C5UV & $\mathrm{C} 5 \mathrm{~V} 3$ & C5UY \\
\hline 2006 & 264.5 & 346.0 & 484.3 & 687.5 & 980.5 & 6.50 & 8.37 & 11.76 & 17.38 & 25.64 \\
\hline 2008 & 283.0 & 371.5 & 522.0 & 737.7 & 1055.5 & 6.96 & 8.95 & 12.63 & 18.69 & 27.54 \\
\hline 2009 & 290.8 & 379.5 & 531.1 & 752.7 & 1080.4 & 7.21 & 9.25 & 13.09 & 19.28 & 28.40 \\
\hline \multicolumn{11}{|c|}{ Female employees } \\
\hline & C5UB & C5UE & $\mathrm{C} 5 \mathrm{UH}$ & C5UK & C5UN & C5UQ & C5UT & C5UW & C5V4 & C5UZ \\
\hline 2006 & 226.3 & 282.1 & 383.3 & 550.0 & 724.9 & 5.98 & 7.42 & 10.16 & 14.92 & 20.39 \\
\hline 2007 & 233.5 & 289.8 & 394.8 & 565.4 & 749.0 & 6.18 & 7.67 & 10.48 & 15.33 & 20.95 \\
\hline 2008 & 241.0 & 302.9 & 412.4 & 590.8 & 777.9 & 6.37 & 7.95 & 10.94 & 16.01 & 21.65 \\
\hline 2009 & 250.4 & 313.0 & 426.4 & 612.9 & 812.2 & 6.63 & 8.28 & 11.42 & 16.62 & 22.64 \\
\hline
\end{tabular}

1 Median values are less affected by extremes of earnings at either ends of Sources: Annual Survey of Hours and Earnings; the scale with half the employees earning above the stated amount and half Office for National Statistics: 01633456120 below.

2 Data relate to full-time employees on adult rates whose pay for the survey pay-period was not affected by absence. 
Full time employees on adult rates whose pay was unaffected by absence

$\begin{array}{lllllll}18-21 & 22-29 & 30-39 & 40-49 & 50-59 & \text { All ages }\end{array}$

Median gross weekly earnings

All

$\begin{array}{ll} & \text { JRG9 } \\ 2006 & 250.6 \\ 2007 & 265.5 \\ 2008 & 271.6 \\ 2009 & 277.7\end{array}$

Men

2006

2007

2008

2009

Women

2006

2007

2008

2009

Median hourly earnings(excluding overtime)

All

2006

2007

2008

2009

Men

2006

2007

2008

2009

Women

2006

2007

2008

2009

268.3

6.64
6.94

$\begin{array}{ll}\mathrm{JRH} 2 & \mathrm{JR} \\ 376.5 & 496.1 \\ 387.8 & 509 . \\ 400.0 & 532.7 \\ 407.5 & 541.7\end{array}$

JRH3 JRH4

502.5

517.3

$\begin{array}{ll}52.7 & 539.9\end{array}$

$\begin{array}{ll}541.7 & 550.6\end{array}$

JRH9

390.6

402.5

416.7

421.6

$\mathrm{JRI} 2$
525.0
539.0
566.3
571.1

JRI8

362.7
374.1

374.1

384.7

392.9

444.0
460.6

480.9

497.5

JRI3

558.7

574.9

599.1

605.9

JRJ2

410.2

420.3

437.3

457.7

JRJ8

12.43

12.43
12.77

13.34

13.80

JRJ9

12.49

12.77

13.31

10.12
10.44

13.84

JRK7

12.78

13.10

13.70

14.15

13.46

13.77

14.37

14.96

\section{JRL6}

11.87

12.28

12.78

13.27

10.12
10.40

9.48

9.48
9.79

1 Median values are less affected by extremes of earnings at either ends of the scale with half the employees earning above the stated amount and half below.

2 Data relate to full-time employees on adult rates whose pay for the survey

pay-period was not affected by absence. 


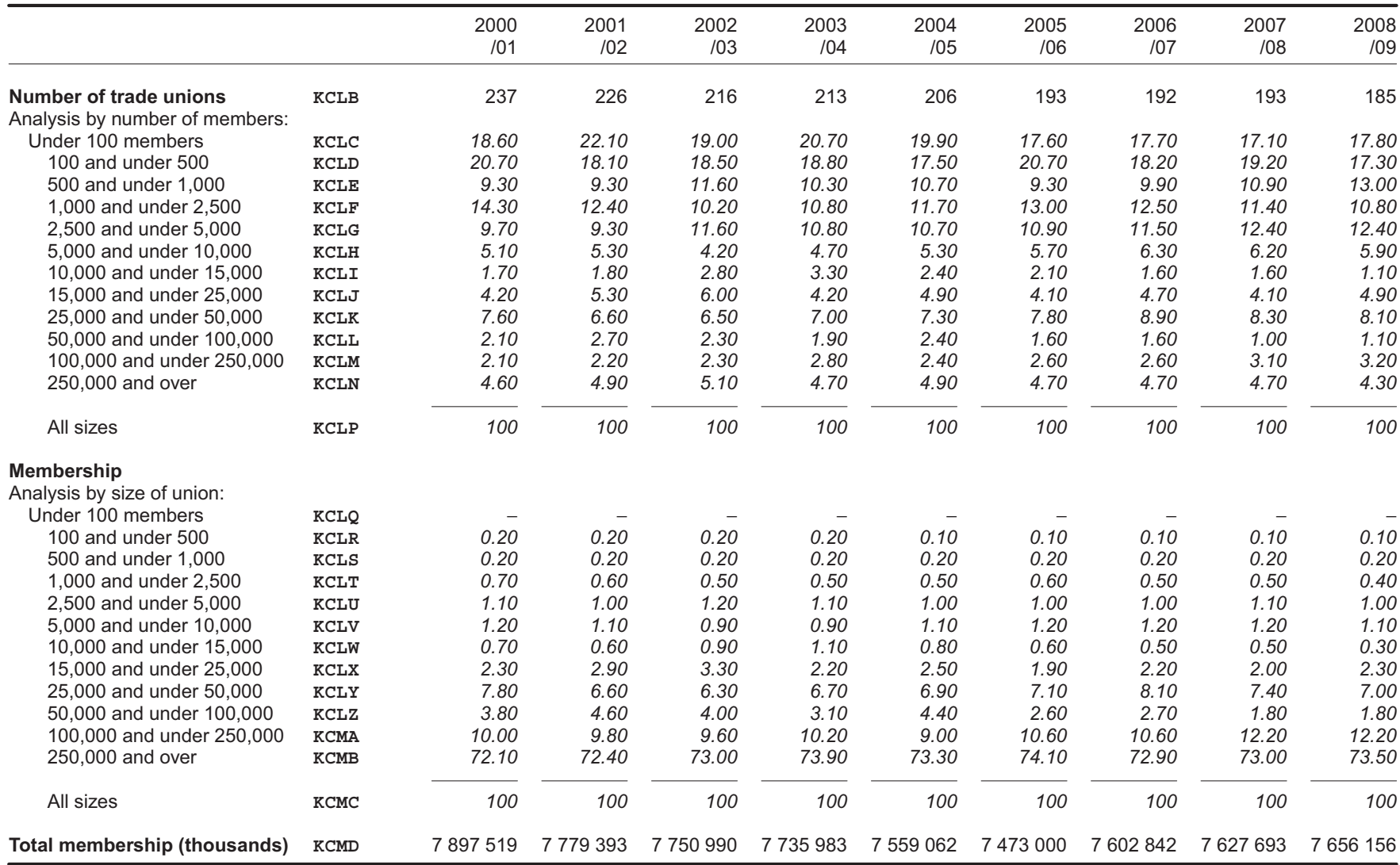

1 See chapter text.

2 Data derived from trade union annual returns with periods which ended between October and September each year. The majority, however, ended in December. In the case of year 2004/05, for example, the data derived from annual returns with periods which ended between October 2004 and September 2005 - approximately $73 \%$ ended in December. 